\title{
EXPONENTIAL-SQUARE INTEGRABILITY, WEIGHTED INEQUALITIES FOR THE SQUARE FUNCTIONS ASSOCIATED TO OPERATORS AND APPLICATIONS
}

\author{
PENG CHEN, XUAN THINH DUONG, LIANGCHUAN WU AND LIXIN YAN
}

\begin{abstract}
Aвstract. Let $X$ be a metric space with a doubling measure. Let $L$ be a nonnegative self-adjoint operator acting on $L^{2}(X)$, hence $L$ generates an analytic semigroup $e^{-t L}$. Assume that the kernels $p_{t}(x, y)$ of $e^{-t L}$ satisfy Gaussian upper bounds and Hölder's continuity in $x$ but we do not require the semigroup to satisfy the preservation condition $e^{-t L} 1=1$. In this article we aim to establish the exponential-square integrability of a function whose square function associated to an operator $L$ is bounded, and the proof is new even for the Laplace operator on the Euclidean spaces $\mathbb{R}^{n}$. We then apply this result to obtain: (i) estimates of the norm on $L^{p}$ as $p$ becomes large for operators such as the square functions or spectral multipliers; (ii) weighted norm inequalities for the square functions; and (iii) eigenvalue estimates for Schrödinger operators on $\mathbb{R}^{n}$ or Lipschitz domains of $\mathbb{R}^{n}$.
\end{abstract}

\section{Contents}

1. Introduction

2. Notations and preliminaries on operators

3. The discrete square function

4. Exponential-square integrability

4.1. John-Nirenberg type inequality for the discrete square function 14

4.2. Exponential-square estimates 18

5. Singular integral operators 26

5.1. Square function results 26

5.2. $L^{p}$ bounds for spectral multipliers $\quad 29$

5.3. Two-weighted norm inequalities for the square function 32

6. Eigenvalue estimates for Schrödinger operators 39

References 42

\section{INTRODUCTION}

1.1. Background. Consider the Laplace operator $\Delta=-\sum_{i=1}^{n} \partial_{x_{i}}^{2}$ on the Euclidean space $\mathbb{R}^{n}$. For a (suitable) function $f(x)$ on $\mathbb{R}^{n}$, the Poisson integral $u(x, t)=e^{-t \sqrt{\Delta}} f(x)$ is the harmonic extension of $f$ to $\mathbb{R}_{+}^{n+1}$. For $\alpha>0$, define the (classical) square function (or classical Lusin area inegral) by

$$
S_{\alpha} f(x)=\left(\iint_{|x-y|<\alpha t}\left|\nabla_{y} u(y, t)\right|^{2} t^{1-n} d y d t\right)^{1 / 2} .
$$

Date: October 11, 2018.

2010 Mathematics Subject Classification. 42B20, 42B25, 47B38, 58G35.

Key words and phrases. Exponential-square integrability, weighted norm inequalities, the square function, the dyadic square function, semigroups, spectral multiplier, eigenvalue estimate, space of homogeneous type. 
It is known that if $S_{\alpha} f$ is a bounded function, then $f$ belongs to the space BMO and hence the JohnNirenberg inequality implies that $f$ is in the exponential class. In other words, $\exp \left(c|f| /\left\|S_{\alpha} f\right\|_{L^{\infty}}\right) \in$ $L_{\text {loc }}^{1}\left(\mathbb{R}^{n}\right)$ for a suitable constant $c>0$. (See [42] for the definition and properties of BMO functions.) Moreover, a celebrated theorem of Chang-Wilson-Wolff ([8, Theorem 3.2]) states that

$$
\exp \left(c|f|^{2} /\left\|S_{\alpha} f\right\|_{L^{\infty}}^{2}\right) \in L_{\mathrm{loc}}^{1}\left(\mathbb{R}^{n}\right)
$$

for a suitable constant $c>0$ depending on $n$ and $\alpha$.

This result (1.2) has been useful in the work of R. Fefferman, C. Kenig and J. Pipher; in the study of Hardy spaces; and also in the work of C. Moore and M. Wilson (see page 486, [7]). Due to R. Fefferman and J. Pipher, it was observed in [23] that (1.2) implies that for the Hilbert transform $H$ (or other singular integrals) on $\mathbb{R}$, as $p \rightarrow \infty$,

$$
\|H f\|_{L^{p}(\mathbb{R})} \leq C p\|f\|_{L^{p}(\mathbb{R})},
$$

which is well-known to be the sharp dependence on $p$. Analogous sharp estimate holds for the square function,

$$
\|f\|_{L^{p}\left(\mathbb{R}^{n}\right)} \leq C p^{1 / 2}\left\|S_{\alpha} f\right\|_{L^{p}\left(\mathbb{R}^{n}\right)}
$$

as $p \rightarrow \infty$ (see page 357, [23]). We note that $\left\|S_{\alpha} f\right\|_{L^{p}\left(\mathbb{R}^{n}\right)} \leq C p^{1 / 2}\|f\|_{L^{p}\left(\mathbb{R}^{n}\right)}$ follows from Theorem 1.2 in [8] with a constant $C$ that depends on $n$ and $\alpha$.

The result (1.2) also has other applications; for example, M. Wilson used it to derive sharp weighted inequalities for the square function to obtain Sobolev and singular integral inequalities and eigenvalue estimates for degenerate Schrödinger operators (see $[46,47,48]$ ). In the last few decades, this result (1.2) has been studied extensively, see for examples, [22, 36, 43, 46, 47, 48] and the references therein.

1.2. Assumptions, notations and definitions. Our setting will be the following. We consider a nonnegative self-adjoint operator $L$ on $L^{2}(X)$ where $(X, d, \mu)$ is a metric measure space which satisfies the volume doubling condition

$$
\mu(B(x, \lambda r)) \leq C \lambda^{n} \mu(B(x, r)), \quad \forall x \in X, \lambda \geq 1, r>0,
$$

where $C$ and $n$ are positive constants and $\mu(B(x, r))$ denotes the volume of the open ball $B(x, r)$ of centre $x$ and radius $r$. In this article, we assume that $L$ is a densely-defined operator on $L^{2}(X)$. In different sections of the article, we assume that $L$ satisfies some or all the following properties:

(H1) $L$ is a nonnegative self-adjoint operator on $L^{2}(X)$;

(H2) The kernel of $e^{-t L}$, denoted by $p_{t}(x, y)$, is a measurable function on $X \times X$ and satisfies a Gaussian upper bound, that is

$$
\left|p_{t}(x, y)\right| \leq \frac{C}{\mu(B(x, \sqrt{t}))} \exp \left(-\frac{d(x, y)^{2}}{c t}\right)
$$

for all $t>0$ and $x, y \in X$, where $C$ and $c$ are positive constants.

(H3) The kernel $p_{t}(x, y)$ satisfies the following Hölder's continuity estimate: there exists a constant $\theta \in(0,1]$, such that

$$
\left|p_{t}(x, y)-p_{t}\left(x^{\prime}, y\right)\right| \leq C\left(\frac{d\left(x, x^{\prime}\right)}{\sqrt{t}}\right)^{\theta} \frac{1}{\mu(B(y, \sqrt{t}))}
$$

for $d\left(x, x^{\prime}\right) \leq \sqrt{t}$. 
The operator $L$ is a nonnegative self-adjoint operator on $L^{2}(X)$, hence $L$ generates the $L$-Poisson semigroup $\left\{e^{-t \sqrt{L}}\right\}_{t>0}$ on $L^{2}(X)$. Given an operator $L$ satisfying (H1) and (H2), and a function $f \in$ $L^{2}(X)$, consider the following square function $S_{L, \alpha} f$ associated to the Poisson semigroup generated by $L$

$$
S_{L, \alpha} f(x):=\left(\int_{0}^{\infty} \int_{d(y, x)<\alpha t}\left|t^{2} L e^{-t \sqrt{L}} f(y)\right|^{2} \frac{d \mu(y)}{\mu(B(x, t))} \frac{d t}{t}\right)^{1 / 2}, \quad \alpha>0,
$$

and set $S_{L} f=S_{L, 1} f$.

It is known that if $L$ satisfies assumptions $(\mathbf{H 1})$ and $(\mathbf{H 2})$, then the operator $S_{L, \alpha}$ is bounded on $L^{p}(X), 1<p<\infty$. (See for example, [1], [25, Proposition 3.3].)

1.3. Main results. This article has two aims. The first one is to address the following question: Is it possible to establish harmonic analysis properties of the exponential-square class of (1.2) on a set? It would be nice to find a proof which does not require the group structure. In this article we will give an affirmative answer to this question by extending the work of Chang, Wilson and Wolff ([8, Theorem 3.2]) for the square function (1.3) on a space of homogeneous type. We have the following result.

Theorem A. Assume that $X$ satisfies the volume doubling condition. Suppose that L is a denselydefined operator on $L^{2}(X)$ satisfying (H1), (H2) and (H3). Let $f \in L^{2}(X, d \mu)$ and assume that $S_{L, \alpha} f \in L^{\infty}(X)$. Then there exist positive constants $\gamma=\gamma(n, \alpha)$ and $C=C(n, \alpha)$ independent of $f$ such that

$$
\sup _{B} \frac{1}{\mu(B)} \int_{B} \exp \left(\gamma \frac{\left|f(x)-f_{B}\right|^{2}}{\left\|S_{L, \alpha} f\right\|_{L^{\infty}(X)}^{2}}\right) d \mu(x) \leq C,
$$

where $f_{B}=\frac{1}{\mu(B)} \int_{B} f(x) d \mu(x)$, and the sup is taken over all balls in $X$.

We mention that the proof of Chang-Wilson-Wolff is using reduction to a related distribution function inequality for dyadic martingales on $\mathbb{R}^{n}$, in which the fundamental identity of sequential analysis in statistics was applied ([8, Theorem 3.1]). In the proof of our Theorem A, the main device is to use some dyadic structures of $X$ to define a version of discrete square functions associated to an operator $L$ on a space $X$ of homogeneous type. From this, we are able to show that boundedness of the discrete square function implies its local exponential-square integrability, and then apply the Calderón machinery ([6]) to allow us to translate the "discrete" results into the continuous setting. Our proof is different substantially from the proof of Chang-Wilson-Wolff which is not applicable in our setting since no group structure is available and the Fourier transform is missing in the general setting of space of homogeneous type. Another observation is that we do not assume that the semigroup $\left\{e^{-t L}\right\}_{t>0}$ satisfies the standard preservation condition, so we may have

$$
e^{-t L} 1 \neq 1
$$

and this allows us to apply the results to a wide class of operators $L$.

We also note that the kernel for $e^{-t L}$ is not assumed to be translation invariant and several techniques which can be used with the Poisson kernel of the Laplacian on $\mathbb{R}^{n}$ are not applicable here. This is indeed one of the main obstacles in this article and makes the theory quite subtle and delicate. We overcome this problem in the proof in Section 4 by using some estimates on heat kernel bounds, finite propagation speed of solutions to the wave equations and spectral theory of nonnegative selfadjoint operators. 
The second aim of this article is to apply (1.4) to give various applications. The first is to obtain estimates of the $L^{p}$ operator norms as $p \rightarrow \infty$ for operators such as the square functions or spectral multipliers. We have the following result.

Theorem B. Assume that $X$ satisfies the volume doubling condition. Assume that $L$ is a denselydefined operator on $L^{2}(X)$ satisfying $(\boldsymbol{H 1}),(\boldsymbol{H} 2)$ and $(\boldsymbol{H} 3)$.

(i) There exists a constant $C=C(n, \alpha)>0$ such that as $p \rightarrow \infty$,

$$
\|f\|_{L^{p}(X)} \leq C p^{1 / 2}\left\|S_{L, \alpha} f\right\|_{L^{p}(X)} .
$$

(ii) Let $\beta>n / 2$ and assume that $F:[0, \infty) \rightarrow \mathbb{C}$ is a bounded Borel function such that $F(0)=0$, $\sup _{t>0}\left\|\eta \delta_{t} F\right\|_{W_{\beta}^{\infty}(\mathbb{R})}<\infty$ where $\delta_{t} F(\lambda)=F(t \lambda)$ and $\eta \in C_{0}^{\infty}\left(\mathbb{R}_{+}\right)$is a fixed function, not identically zero. Then there exists a constant $C=C(n, \beta)>0$ such that the operator $F(L)$ satisfies

$$
\|F(L) f\|_{L^{p}(X)} \leq C p \sup _{t>0}\left\|\eta \delta_{t} F\right\|_{W_{\beta}^{\infty}(\mathbb{R})}\|f\|_{L^{p}(X)}
$$

as $p \rightarrow \infty$.

We remark that under the assumptions (H1) and (H2) of the operator $L$, it is known that there exists a constant $C=C(n, \alpha)>0$ such that

$$
\left\|S_{L, \alpha} f\right\|_{L^{p}(X)} \leq C p^{1 / 2}\|f\|_{L^{p}(X)}
$$

as $p \rightarrow \infty$ (see $[25,24])$. Combining this with (i) of Theorem $\mathrm{B}$, we then obtain the estimate

$$
c p^{-1 / 2}\|f\|_{L^{p}(X)} \leq\left\|S_{L, \alpha} f\right\|_{L^{p}(X)} \leq C p^{1 / 2}\|f\|_{L^{p}(X)}
$$

as $p \rightarrow \infty$.

Another application is to apply (1.4) to obtain a two weighted inequality for the square function $S_{L, \alpha}$ associated to an operator $L$ (see Theorem 5.6 below for precise statement).

Theorem C. Assume that $X$ satisfies the volume doubling condition and $L$ is a densely-defined operator on $L^{2}(X)$ satisfying $(\boldsymbol{H 1}),(\boldsymbol{H} 2)$ and $(\boldsymbol{H} 3)$. Let $0<p<\infty, \tau>p / 2$, and let $V$ and $W$ be two weights such that

$$
\int_{Q} V(x)\left(\log \left(e+\frac{V(x)}{V_{Q}}\right)\right)^{\tau} d \mu(x) \leq \int_{Q} W(x) d \mu(x)
$$

for all $Q \in \mathcal{D}$. Then for all $\alpha>0$, there exists a constant $C=C(p, n, \tau, \alpha)>0$ such that for every $f \in L_{0}^{\infty}(X)$,

$$
\int_{X}|f(x)|^{p} V(x) d \mu(x) \leq C \int_{X}\left(S_{L, \alpha} f(x)\right)^{p} W(x) d \mu(x) .
$$

Finally, we study the eigenvalue problem of Schrödinger operators on $\mathbb{R}^{n}$ or a Lipschitz domain of $\mathbb{R}^{n}$. In the following, we let $\Omega=\mathbb{R}^{n}$ or $\Omega$ be a special Lipschitz domain of $\mathbb{R}^{n}$. Let $A(x)$ be an $n \times n$ matrix function with real symmetric, bounded measurable entries on $\mathbb{R}^{n}$ satisfying the ellipticity condition

$$
\|A\|_{\infty} \leq \lambda^{-1} \text { and } A(x) \xi \cdot \xi \geq \lambda|\xi|^{2}
$$

for some constant $\lambda \in(0,1)$, for all $\xi \in \Omega$ and for almost all $x \in \Omega$. For a nonnegative $V$ in $L_{\text {loc }}^{1}(\Omega)$, we consider the Schrödinger operator $\mathcal{L}$, defined by

$$
\mathcal{L} u=-\operatorname{div}(A(x) \nabla u)-V u .
$$


We note that the spectrum of the operator $L=-\operatorname{div} A \nabla$ is contained in $[0, \infty)$. However, the effect of the potential $V \geq 0$ is that the spectrum of $\mathcal{L}$ might contain some negative values. We have the following result.

Theorem D. When $\Omega=\mathbb{R}^{n}$ or $\Omega$ is a special Lipschitz domain of $\mathbb{R}^{n}$, let $\mathcal{L}=-\operatorname{div}(A \nabla)-V$ be a real symmetric operator (under Dirichlet boundary condition or Neumann boundary condition) with nonnegative $V \in L_{\text {loc }}^{1}(\Omega)$ as in (1.5). Let $\sigma(\mathcal{L})$ be the lowest nonpositive eigenvalue of $\mathcal{L}$ on $\Omega$. Fix $\tau>1$. For every ball $B=B\left(x_{B}, r_{B}\right)$ in $\Omega$, define

$$
\Lambda(B, V)=\int_{B} V(x)\left(\log \left(e+\frac{V(x)}{V_{B}}\right)\right)^{\tau} d \mu(x) .
$$

Then there exist two positive constants $c_{1}$ and $c_{2}$ which depend on $n, \tau$ such that

$$
\sigma(\mathcal{L}) \geq-\sup _{B} c_{1}\left[|B|^{-1} \Lambda(B, V)-c_{2} r_{B}^{-2}|B|\right],
$$

where $|B|$ denotes the Lebesgue measure of $B$ in $\Omega$.

As a consequence, if

$$
r_{B}^{2} \Lambda(B, V) \leq c_{2}|B|^{2}
$$

for all balls $B$ in $\Omega$, then $\mathcal{L}$ is nonnegative.

We mention that in the Euclidean spaces $\mathbb{R}^{n}$, such operators have received a great deal of attention in the past decades. In particular, the case $A=I$, the identity, was studied, see for example, in $[9,8,21,31,36]$ and the references therein; while the case of nonconstant $A$ was treated in [46, 47].

The layout of the article is as follows. In Section 2 we recall some basic properties of space of homogeneous type, heat kernels and finite propagation speed for the wave equation, and build the necessary kernel estimates for functions of an operator. In Section 3 we introduce the discrete square function on space of homogeneous type. In Section 4 we give a proof of Theorem A by using reduction to a version of discrete square function on space of homogeneous type. Theorems B, C and $\mathrm{D}$ will be proved in Sections 5 and 6, which include estimates of the $L^{p}$ operator norms as $p \rightarrow \infty$ for operators such as the square functions or spectral multipliers, weighted norm inequalities for the square functions and eigenvalue estimates for Schrödinger operators on $\mathbb{R}^{n}$ or on a special Lipschitz domain of $\mathbb{R}^{n}$.

\section{NOTATIONS AND PRELIMINARIES ON OPERATORS}

We start by introducing some notations and assumptions. Throughout this article, unless we mention the contrary, $(X, d, \mu)$ is a metric measure space, that is, $\mu$ is a Borel measure with respect to the topology defined by the metric $d$. We denote by $B(x, r)=\{y \in X, d(x, y)<r\}$ the open ball with centre $x \in X$ and radius $r>0$. We often just use $B$ instead of $B(x, r)$. Given $\lambda>0$, we write $\lambda B$ for the $\lambda$-dilated ball which is the ball with the same centre as $B$ and radius $\lambda r$. We say that $(X, d, \mu)$ satisfies the doubling property (see [13, Chapter 3]) if there exists a constant $C>0$ such that

$$
\mu(B(x, 2 r)) \leq C \mu(B(x, r)), \quad \forall r>0, x \in X .
$$

If this is the case, there exist $C, n$ such that for all $\lambda \geq 1$ and $x \in X$

$$
\mu(B(x, \lambda r)) \leq C \lambda^{n} \mu(B(x, r)) \text {. }
$$

In the Euclidean space with Lebesgue measure, $n$ corresponds to the dimension of the space.

Throughout the article, we will suppose that $\mu(X)=\infty$ and $\mu(\{x\})=0$ for all $x \in X$. For $1 \leq p \leq+\infty$, we denote the norm of a function $f \in L^{p}(X, \mathrm{~d} \mu)$ by $\|f\|_{p}$, by $\langle\cdot, \cdot\rangle$ the scalar product of $L^{2}(X, \mathrm{~d} \mu)$, and if $T$ is a bounded linear operator from $L^{p}(X, \mathrm{~d} \mu)$ to $L^{q}(X, \mathrm{~d} \mu), 1 \leq p, q \leq+\infty$, 
we write $\|T\|_{p \rightarrow q}$ for the operator norm of $T$. Given a subset $E$, we denote by $\chi_{E}$ the characteristic function of $E$. For every $f \in L_{\mathrm{loc}}^{1}(X)$ and a subset $E$, we write

$$
f_{E}=(f)_{E}=\frac{1}{\mu(E)} \int_{E} f(x) d \mu(x), \quad \text { and } \quad|f|_{E}=\frac{1}{\mu(E)} \int_{E}|f(x)| d \mu(x) .
$$

For $F \in L^{1}(\mathbb{R})$, the Fourier transform of $F$ is given by

$$
\widehat{F}(t)=\frac{1}{2 \pi} \int_{-\infty}^{+\infty} F(\lambda) e^{-i t \lambda} d \lambda .
$$

Finally, $C$ and $c$ denote two generic constants, not necessarily the same at each occurrence, which, in the course of a proof, may be taken to depend on any of the non-essential quantities assumed to be bounded.

2.1. System of dyadic cubes. In a metric space $(X, d)$, a countable family

$$
\mathscr{D}=\bigcup_{k \in \mathbb{Z}} \mathscr{D}_{k}, \quad \mathscr{D}_{k}=\left\{Q_{\beta}^{k}: \beta \in \mathscr{J}_{k}\right\}
$$

of Borel sets $Q_{\beta}^{k} \subset X$ is called a system of dyadic cubes with parameter $\delta \in(0,1)$ if it satisfies the following properties:

(i) $X=\bigcup_{\beta \in \mathscr{J}_{k}} Q_{\beta}^{k}$ (disjoint union) for all $k \in \mathbb{Z}$;

(ii) if $\ell \geq k$, then either $Q_{\beta_{2}}^{\ell} \subseteq Q_{\beta_{1}}^{k}$ or $Q_{\beta_{1}}^{k} \cap Q_{\beta_{2}}^{\ell}=\emptyset$;

(iii) for each $\left(k, \beta_{1}\right)$ and each $\ell \leq k$, there exists a unique $\beta_{2}$ such that $Q_{\beta_{1}}^{k} \subseteq Q_{\beta_{2}}^{\ell}$;

(iv) there exists a positive constant $M=M(n, \delta)$ such that for each $\left(k, \beta_{1}\right)$,

$$
1 \leq \#\left\{\beta_{2}: Q_{\beta_{2}}^{k+1} \subseteq Q_{\beta_{1}}^{k}\right\} \leq M \quad \text { and } \quad Q_{\beta_{1}}^{k}=\bigcup_{\beta_{2}: Q_{\beta_{2}}^{k+1} \subseteq Q_{\beta_{1}}^{k}} Q_{\beta_{2}}^{k+1}
$$

(v) for each $(k, \beta)$,

$$
B\left(x_{\beta}^{k}, \delta^{k} / 3\right) \subseteq Q_{\beta}^{k} \subseteq B\left(x_{\beta}^{k}, 2 \delta^{k}\right)=: B\left(Q_{\beta}^{k}\right)
$$

for some $x_{\beta}^{k} \in Q_{\beta}^{k}$;

(vi) if $\ell \geq k$ and $Q_{\beta_{2}}^{\ell} \subseteq Q_{\beta_{1}}^{k}$, then $B\left(Q_{\beta_{2}}^{\ell}\right) \subset B\left(Q_{\beta_{1}}^{k}\right)$.

The set $Q_{\beta}^{k}$ is called a dyadic cube of generation $k$ with center point $x_{\beta}^{k} \in Q_{\beta}^{k}$. For $Q \in \mathscr{D}_{k}$, for every $\ell \geq 0$ we denote by

$$
C h^{(\ell)}(Q)=\left\{P \subset Q: P \in \mathscr{D}_{k+\ell}\right\} .
$$

Obviously, $C h^{(0)}(Q)=\{Q\}$ and $C h^{(1)}(Q)=C h(Q)$, which is called the children of $Q$.

Next, we recall the following construction due to T. Hytönen and A. Kairema [30], which is a slight elaboration of seminal work by M. Christ [12], as well as E. Sawyer and R.L. Wheeden [38].

Proposition 2.1. Let $(X, d)$ be a metric space. Then there exists a system of dyadic cubes with parameter $0<\delta<1 / 144$. The construction only depends on a fixed set of countably many points $x_{\beta}^{k}$, having the properties that

$$
d\left(x_{\beta_{1}}^{k}, x_{\beta_{2}}^{k}\right) \geq \delta^{k}\left(\beta_{1} \neq \beta_{2}\right), \quad \min _{\beta} d\left(x, x_{\beta}^{k}\right)<\delta^{k} \text { for all } x \in X,
$$

and a partial order " $\leq$ " among the pairs $(k, \beta)$ such that

- if $d\left(x_{\beta_{2}}^{k+1}, x_{\beta_{1}}^{k}\right)<\delta^{k} / 6$, then $\left(k+1, \beta_{2}\right) \leq\left(k, \beta_{1}\right)$;

- if $\left(k+1, \beta_{2}\right) \leq\left(k, \beta_{1}\right)$, then $d\left(x_{\beta_{2}}^{k+1}, x_{\beta_{1}}^{k}\right)<2 \delta^{k}$;

- for every $\left(k+1, \beta_{2}\right)$, there is exactly one $\left(k, \beta_{1}\right) \geq\left(k+1, \beta_{2}\right)$, called its parent; 
- for every $\left(k, \beta_{1}\right)$, there are between 1 and M pairs $\left(k+1, \beta_{2}\right) \leq\left(k, \beta_{1}\right)$, called its children.

We will refer the sets $\left\{x_{\beta}^{k}\right\}_{k, \beta \in \mathscr{J}_{k}}$ of dyadic points as the set of reference points. Now fix a set of reference points $\left\{x_{\beta}^{k}: k \in \mathbb{Z}, \beta \in \mathscr{J}_{k}\right\}$ and some $0<\delta<1 / 144$. From [30, Section 4], reference points $x_{\beta_{1}}^{k}$ and $x_{\beta_{2}}^{k}, \beta_{1} \neq \beta_{2}$, of the same generation are in conflict if $d\left(x_{\beta_{1}}^{k}, x_{\beta_{2}}^{k}\right)<\delta^{k-1}$. Reference points $x_{\beta_{1}}^{k}$ and $x_{\beta_{2}}^{k}, \beta_{1} \neq \beta_{2}$, of the same generation are neighbors if there occurs a conflict between their children. It is known that for every reference point $x_{\beta}^{k}$, the number of neighbors of $x_{\beta}^{k}$ is bounded from above by a fixed constant, say $N$. Then every point $x_{\beta_{1}}^{k}$ gets a primary label

$$
\operatorname{label}_{1}\left(k, \beta_{1}\right):=\ell
$$

not bigger than $N=N(n, \delta)$, such that if $\left(k, \beta_{1}\right)$ and $\left(k, \beta_{2}\right), \beta_{1} \neq \beta_{2}$, have the same label $\ell \in$ $\{0,1, \cdots, N\}$, they are not neighbors. Next we label the reference points $x_{\beta}^{k+1}$ of the next generation $k+1$ with duplex labels: If $\boldsymbol{l a b e l}_{\mathbf{1}}\left(k, \beta_{1}\right)=\ell$, each of its children $\left(k+1, \beta_{2}\right) \leq\left(k, \beta_{1}\right)$ gets a different duplex label

$$
\text { label }_{\mathbf{2}}\left(k+1, \beta_{2}\right):=(\ell, m), \quad m\left(\beta_{2}\right) \in\{1,2, \cdots, M\} .
$$

Then adjacent dyadic systems are constructed by the following specific selection rule: Fix $(\ell, m) \in$ $\{0, \cdots, N\} \times\{1, \cdots, M\}$. For every index pair $\left(k, \beta_{1}\right)$, check whether there exists $\left(k+1, \beta_{2}\right) \leq\left(k, \beta_{1}\right)$ with label pair $(\ell, m)$. If so, decree that $z_{\beta_{1}}^{k}:=x_{\beta_{2}}^{k+1}$. Otherwise, pick some $\left(k+1, \beta_{2}\right) \leq\left(k, \beta_{1}\right)$ with $\rho\left(x_{\beta_{2}}^{k+1}, x_{\beta_{1}}^{k}\right)<\delta^{k+1}$ and decree $z_{\beta_{1}}^{k}:=x_{\beta_{2}}^{k+1}$. Define

$$
\begin{aligned}
\Lambda:\{0, \cdots, N\} \times\{1, \cdots, M\} & \rightarrow\{1, \cdots, K\} \subset \mathbb{N} \\
(\ell, m) & \mapsto b
\end{aligned}
$$

which is a bijection. We identify $b=\Lambda(\ell, m)$ with $(\ell, m)$. Each $b$ gives rise to a set $\left\{{ }^{b} z_{\beta}^{k}: k \in \mathbb{Z}, \beta \in\right.$ $\left.\mathscr{J}_{k}\right\}$ of new dyadic points associated with the duplex label $(\ell, m)=b$. From these new dyadic points $\left\{{ }^{b} z_{\beta}^{k}: k \in \mathbb{Z}, \beta \in \mathscr{J}_{k}\right\}$, we can construct adjacent dyadic systems

$$
\left\{\mathcal{D}^{b}: b=1,2, \cdots, K\right\},
$$

where $\mathcal{D}^{b}=\bigcup_{k \in \mathbb{Z}} \mathcal{D}_{k}^{b}, \mathcal{D}_{k}^{b}=\left\{{ }^{b} Q_{\beta}^{k}: \beta \in \mathscr{J}_{k}\right\}$, and

$$
B\left({ }^{b} z_{\beta}^{k}, \delta^{k} / 12\right) \subset{ }^{b} Q_{\beta}^{k} \subset B\left({ }^{b} z_{\beta}^{k}, 4 \delta^{k}\right) .
$$

For more details, we refer the reader to [30, Section 4], and in the sequel, we assume $0<\delta<1 / 144$.

Throughout the article, if ${ }^{b} Q_{\beta_{1}}^{k} \in \mathcal{D}^{b}$ has only one child, namely ${ }^{b} Q_{\beta_{2}}^{k+1}$, although ${ }^{b} Q_{\beta_{1}}^{k}$ and ${ }^{b} Q_{\beta_{2}}^{k+1}$ are the same set of points, ${ }^{b} Q_{\beta_{1}}^{k}$ and ${ }^{b} Q_{\beta_{2}}^{k+1}$ are seen as two different cubes. Then we have the following proposition.

Proposition 2.2. There exists an injective mapping

$$
E_{2}: \mathscr{D} \rightarrow \mathcal{D}=\bigcup_{b=1}^{K} \mathcal{D}^{b},
$$

such that for every $Q_{\beta}^{k} \in \mathscr{D}, E_{2}\left(Q_{\beta}^{k}\right) \in \mathcal{D}_{k-1}^{b}$ for some $b \in\{1,2, \cdots, K\}$ with the property:

$$
3 Q_{\beta}^{k}:=\left\{y \in X: d\left(y, Q_{\beta}^{k}\right) \leq \delta^{k}\right\} \subset E_{2}\left(Q_{\beta}^{k}\right), \quad \mu\left(E_{2}\left(Q_{\beta}^{k}\right)\right) \leq C \mu\left(Q_{\beta}^{k}\right)
$$

for some constant $C=C(n)$ independent of $(\beta, k)$. 
Proof. Observe that for every $Q_{\beta_{1}}^{k} \in \mathscr{D},\left(k, \beta_{1}\right)$ has a unique $\boldsymbol{l a b e l}_{\mathbf{2}}\left(k, \beta_{1}\right)=(\ell, m)$, and there exists a unique $\beta_{2} \in \mathscr{J}_{k-1}$ such that $\left(k, \beta_{1}\right) \leq\left(k-1, \beta_{2}\right)$. We set $b=\Lambda\left(\right.$ label $\left._{2}\left(k, \beta_{1}\right)\right)$, where $\Lambda$ is the mapping in (2.8). According to the selection rule, there exists ${ }^{b} Q_{\beta_{2}}^{k-1} \in \mathcal{D}^{b}$ with center point ${ }^{b} z_{\beta_{2}}^{k-1}=x_{\beta_{1}}^{k}$. Define

$$
\begin{aligned}
E_{2}: \mathscr{D} & \rightarrow \bigcup_{b=1}^{K} \mathcal{D}^{b}=\bigcup_{b=1}^{K} \bigcup_{k \in \mathbb{Z}} \mathcal{D}_{k}^{b} \\
Q_{\beta_{1}}^{k} & \mapsto{ }^{b} Q_{\beta_{2}}^{k-1}, \quad b=\Lambda\left(\boldsymbol{l a b e l}_{2}\left(k, \beta_{1}\right)\right), \quad\left(k, \beta_{1}\right) \leq\left(k-1, \beta_{2}\right) .
\end{aligned}
$$

Obviously, for every $Q_{\beta_{1}}^{k} \in \mathscr{D}$ in level $k$ with center $x_{\beta_{1}}^{k}$, there exists a unique $b \in\{1,2, \cdots, K\}$ such that $E_{2}\left(Q_{\beta_{1}}^{k}\right)={ }^{b} Q_{\beta_{2}}^{k-1} \in \mathcal{D}_{k-1}^{b}$. It follows from (2.10) that

$$
B\left(x_{\beta_{1}}^{k}, \delta^{k-1} / 12\right) \subseteq E_{2}\left(Q_{\beta_{1}}^{k}\right)={ }^{b} Q_{\beta_{2}}^{k-1} \subseteq B\left(x_{\beta_{1}}^{k}, 4 \delta^{k-1}\right),
$$

and one can apply (2.4) and $\delta<1 / 144$ to deduce

$$
B\left(x_{\beta_{1}}^{k}, \delta^{k} / 3\right) \subset Q_{\beta_{1}}^{k} \subset 3 Q_{\beta_{1}}^{k}=\left\{y \in X: d\left(y, Q_{\beta_{1}}^{k}\right) \leq \delta^{k}\right\} \subset B\left(x_{\beta_{1}}^{k}, 3 \delta^{k}\right) \subset B\left(x_{\beta_{1}}^{k}, \delta^{k-1} / 12\right) .
$$

The doubling property (2.1) implies that there exists a constant $C=C(n)$ such that $\mu\left(E_{2}\left(Q_{\beta_{1}}^{k}\right)\right) \leq$ $C \mu\left(Q_{\beta_{1}}^{k}\right)$ for every $Q_{\beta_{1}}^{k} \in \mathscr{D}$.

Next, we show that $E_{2}$ is injective. Indeed, for any $Q_{\beta_{3}}^{k}, Q_{\beta_{4}}^{k} \in \mathscr{D}$ with $\beta_{3} \neq \beta_{4}$, then $E_{2}\left(Q_{\beta_{3}}^{k}\right) \neq$ $E_{2}\left(Q_{\beta_{4}}^{k}\right)$ since their center points are different. For any $Q_{\beta_{5}}^{k}, Q_{\beta_{6}}^{\ell} \in \mathscr{D}$ with $k \neq \ell$, even though $E_{2}\left(Q_{\beta_{5}}^{k}\right)$ and $E_{2}\left(Q_{\beta_{6}}^{\ell}\right)$ are both in some $\mathcal{D}^{b}, b \in\{1,2, \cdots, K\}$, one can see $E_{2}\left(Q_{\beta_{5}}^{k}\right) \in \mathcal{D}_{k-1}^{b}$, while $E_{2}\left(Q_{\beta_{6}}^{\ell}\right) \in \mathcal{D}_{\ell-1}^{b}$. Hence, the proof of Proposition 2.2 is end.

We would like to mention that in the case when $X=\mathbb{R}^{n}$, a cube $Q \subset \mathbb{R}^{n}$ is dyadic if it is of the form

$$
Q=\left(\frac{j_{1}}{2^{k}}, \frac{j_{1}+1}{2^{k}}\right) \times \cdots \times\left(\frac{j_{n}}{2^{k}}, \frac{j_{n}+1}{2^{k}}\right)
$$

for some integers $k$ and $j_{i}, i=1, \cdots, n$. We denote the collection of all dyadic cubes by $\mathscr{D}$. Let $\mathcal{D}$ be the collection of all triples of dyadic cubes. It is known ([47, Lemma 2.1]) that there exist disjoint families $\mathcal{D}^{1}, \cdots, \mathcal{D}^{3^{n}}$ such that $\mathcal{D}=\cup_{b=1}^{3^{n}} \mathcal{D}^{b}$. In this case, one can define the mapping $E_{2}: \mathscr{D} \rightarrow \bigcup_{b=1}^{3^{n}} \mathcal{D}^{b}$ in (2.11) as follows:

$$
E_{2}(Q)=3 Q
$$

for every $Q \in \mathscr{D}$. Here $3 Q$ denotes the cube concentric with $Q$ while with sidelength three times as big.

2.2. Finite propagation speed property for wave equation. Suppose that $L$ is a densely-defined operator on $L^{2}(X)$. It is known (see for instance $[10,14,39]$ that if $L$ satisfies (H1) and (H2), then $L$ satisfies the finite propagation speed property, that is, there exists a finite, positive constant $c_{0}$ with the property that the Schwartz kernel $K_{\cos (t \sqrt{L})}$ of $\cos (t \sqrt{L})$ satisfies

$$
\operatorname{supp} K_{\cos (t \sqrt{L})} \subset\left\{(x, y) \in X \times X: d(x, y) \leq c_{0} t\right\} .
$$

The precise value of $c_{0}$ is non-essential, and throughout the article we will choose $c_{0}=1$. By the Fourier inversion formula, whenever $F$ is an even bounded Borel function with $\widehat{F} \in L^{1}(\mathbb{R})$, we can write $F(\sqrt{L})$ in terms of $\cos (t \sqrt{L})$. Concretely, we have

$$
F(\sqrt{L})=(2 \pi)^{-1} \int_{-\infty}^{\infty} \widehat{F}(t) \cos (t \sqrt{L}) d t,
$$


which, combined with condition (FS), gives

$$
K_{F(\sqrt{L})}(x, y)=(2 \pi)^{-1} \int_{d(x, y) \leq|t|} \widehat{F}(t) K_{\cos (t \sqrt{L})}(x, y) d t .
$$

Lemma 2.3. Suppose that $L$ is a densely-defined operator on $L^{2}(X)$ satisfying $(\boldsymbol{H 1})$ and (H2). Let $\rho>0, \phi \in C_{0}^{\infty}(\mathbb{R})$ be even, supp $\phi \in(-\rho, \rho)$ and $\varphi=\widehat{\phi}$. For every $t>0$ and $k=0,1,2, \cdots$, $\psi_{k}(t \sqrt{L})=\left(t^{2} L\right)^{k} \varphi(t \sqrt{L})$ is defined by the spectral theory. Then for every $k=0,1,2, \cdots$, we have

(i) The kernel $K_{\psi_{k}(t \sqrt{L})}(x, y)$ of $\psi_{k}(t \sqrt{L})$ satisfies

$$
\operatorname{supp} K_{\psi_{k}(t \sqrt{L})} \subset\{(x, y) \in X \times X: d(x, y) \leq \rho t\}
$$

and

$$
\left|K_{\psi_{k}(t \sqrt{L})}(x, y)\right| \leq \frac{C(k, \rho)}{\mu(B(x, t))}, \quad \forall t>0, \quad \forall x, y \in X ;
$$

(ii) The kernel $K_{\psi_{k}(s \sqrt{L}) \psi_{k}(t \sqrt{L})}(x, y)$ of $\psi_{k}(s \sqrt{L}) \psi_{k}(t \sqrt{L})$ satisfies

$$
\left|K_{\psi_{k}(s \sqrt{L}) \psi_{k}(t \sqrt{L})}(x, y)\right| \leq \min \left(\frac{s}{t}, \frac{t}{s}\right) \frac{C(k, n, \rho)}{\mu(B(x, \max (s, t)))}, \quad \forall s, t>0, \quad \forall x, y \in X ;
$$

(iii) In addition, we assume that $L$ satisfies condition $(\mathbf{H 3})$ with some index $\theta \in(0,1]$. Then the kernel $K_{\psi_{k}(t \sqrt{L})}(x, y)$ of $\psi_{k}(t \sqrt{L})$ satisfies

$$
\left|K_{\psi_{k}(t \sqrt{L})}(x, y)-K_{\psi_{k}(t \sqrt{L})}\left(x^{\prime}, y\right)\right| \leq C(k, n, \rho) \min \left\{\left(\frac{d\left(x, x^{\prime}\right)}{t}\right)^{\theta}, 1\right\} \frac{1}{\mu(B(y, t))}
$$

for all $x, x^{\prime}, y \in X$ and all $t>0$.

Proof. The proof of (i) is standard; see for example, [27, 39]. The proof of (ii) can be obtained by a minor modification with that of [40, Lemma 2.3], and we skip it here.

We now prove (iii). For every $m \geq n / 2+1$ and every $k \in \mathbb{N}$, we write $\eta_{m, k}(u)=\left(1+u^{2}\right)^{m} \psi_{k}(u)$. Then the kernel $K_{\eta_{m, k}(t \sqrt{L})}(x, y)$ of $\eta_{m, k}(t \sqrt{L})$ satisfies property (i) above. On the other hand, we rewrite $\psi_{k}(u)=\left(1+u^{2}\right)^{-m} \eta_{m, k}(u)$. Observe that for any $m \in \mathbb{N}$, we have the relationship

$$
\left(I+t^{2} L\right)^{-m}=\frac{1}{(m-1) !} \int_{0}^{\infty} e^{-s t^{2} L} e^{-s} s^{m-1} d s
$$

To show (2.14), we consider two cases.

Case (1): $d\left(x, x^{\prime}\right) \leq t$.

In this case, we use formula (2.15), together with property (i) of this lemma, (2.2) and condition (H3) to obtain

$$
\begin{aligned}
& \left|K_{\psi_{k}(t \sqrt{L})}(x, y)-K_{\psi_{k}(t \sqrt{L})}\left(x^{\prime}, y\right)\right| \\
& \leq \quad C \int_{X}\left(\int_{0}^{\infty} e^{-s} s^{m-1}\left|p_{t^{2} s}(x, z)-p_{t^{2} s}\left(x^{\prime}, z\right)\right| d s\right)\left|K_{\left(I+t^{2} L\right)^{m} \psi_{k}(t \sqrt{L})}(z, y)\right| d \mu(z) \\
& \leq \quad C(k, \rho) \int_{d(z, y) \leq \rho t} \frac{1}{\mu(B(z, t))} \int_{0}^{\left(d\left(x, x^{\prime}\right) / t\right)^{2}} \frac{1}{\mu(B(z, \sqrt{s} t))} e^{-s} s^{m-1} d s d \mu(z) \\
& \quad+C(k, \rho) \int_{d(z, y) \leq \rho t} \frac{1}{\mu(B(z, t))} \int_{\left(d\left(x, x^{\prime}\right) / t\right)^{2}}^{\infty}\left(\frac{d\left(x, x^{\prime}\right)}{\sqrt{s} t}\right)^{\theta} \frac{1}{\mu(B(z, \sqrt{s} t))} e^{-s} s^{m-1} d s d \mu(z) \\
& =I+I I .
\end{aligned}
$$


For term $I$, we use (2.2) and elementary integration, to verify that for $m \geq n / 2+1$,

$$
\begin{aligned}
I & \leq C(k, \rho) \frac{1}{\mu(B(y, t))} \int_{d(z, y) \leq \rho t} \frac{1}{\mu(B(z, t))} d \mu(z) \int_{0}^{\left(d\left(x, x^{\prime}\right) / t\right)^{2}} s^{-n / 2} e^{-s} s^{m-1} d s \\
& \leq C(k, n, \rho)\left(\frac{d\left(x, x^{\prime}\right)}{t}\right)^{2 m-n} \frac{1}{\mu(B(y, t))} .
\end{aligned}
$$

Now for the term $I I$,

$$
\begin{aligned}
I I & \leq C(k, \rho)\left(\frac{d\left(x, x^{\prime}\right)}{t}\right)^{\theta} \frac{1}{\mu(B(y, t))} \int_{d(z, y) \leq \rho t} \frac{1}{\mu(B(z, t))} d \mu(z) \int_{\left(d\left(x, x^{\prime}\right) / t\right)^{2}}^{\infty} e^{-s} s^{m-1} d s \\
& \leq C(k, n, \rho)\left(\frac{d\left(x, x^{\prime}\right)}{t}\right)^{\theta} \frac{1}{\mu(B(y, t))}
\end{aligned}
$$

which yields (2.14) in this case $d\left(x, x^{\prime}\right) \leq t$.

Case (2): $d\left(x, x^{\prime}\right)>t$.

In this case we apply property (i) of this lemma to see that

$$
\begin{aligned}
\left|K_{\psi_{k}(t \sqrt{L})}(x, y)-K_{\psi_{k}(t \sqrt{L})}\left(x^{\prime}, y\right)\right| & \leq\left|K_{\psi_{k}(t \sqrt{L})}(x, y)\right|+\left|K_{\psi_{k}(t \sqrt{L})}\left(x^{\prime}, y\right)\right| \\
& \leq \frac{C(k, \rho)}{\mu(B(y, t))},
\end{aligned}
$$

which gives our desired estimate. This proves (iii) and completes the proof of Lemma 2.3.

In the end of this section, we mention that our assumptions (H1), (H2) and (H3) on $L$ hold for large variety of second order self-adjoint operators. We list some of them:

1) Let $A(x)$ be an $n \times n$ matrix function with real symmetric, bounded measurable entries on $\mathbb{R}^{n}$ satisfying the ellipticity condition

$$
\|A\|_{\infty} \leq \lambda^{-1} \text { and } A(x) \xi \cdot \xi \geq \lambda|\xi|^{2}
$$

for some constant $\lambda \in(0,1)$, for all $\xi \in \mathbb{R}^{n}$ and for almost all $x \in \mathbb{R}^{n}$. Let $L$ be a second order elliptic operator in divergence form $L=-\operatorname{div}(A \nabla)$ on $L^{2}\left(\mathbb{R}^{n}\right)$. Then properties (H1), (H2) and (H3) for operator $L$ are always satisfied, see $[4,15,35]$.

2) Let $L=-\Delta+V$ be some Schrödinger operator on $\mathbb{R}^{n}, n \geq 3$, where $V$ is a nonnegative potential and belongs to the reverse Hölder class $(R H)_{q}$ for some $q \geq n / 2$, i.e.,

$$
\left(\frac{1}{|B(x, r)|} \int_{B(x, r)} V(y)^{q} d y\right)^{1 / q} \leq \frac{C}{|B(x, r)|} \int_{B(x, r)} V(y) d y,
$$

where $|B(x, r)|$ denotes the Lebesgue measure of ball $B(x, r)$. It's well known that $L$ satisfies (H1), (H2) and (H3), see [20, 37].

3) Consider a complete Riemannian manifold $M$ with the doubling property. One defines $\Delta$, the Laplace-Beltrami operator, as a self-adjoint positive operator on $L^{2}(M)$. If $M$ satisfies suitable geometric conditions, e.g. $M$ has nonnegative Ricci curvature, then $\Delta$ satisfies properties (H1), (H2) and (H3), see [2, 34, 35]. 


\section{THE DISCRETE SQUARE FUNCTION}

In this section, we assume that $X$ satisfies the volume doubling condition, and $L$ is a denselydefined operator on $L^{2}(X)$ satisfying (H1), (H2) and (H3). For a fixed $\rho>0$, we let $\phi \in C_{0}^{\infty}(\mathbb{R})$ be real-valued and even, supp $\phi \in(-\rho, \rho)$, and set $\psi(x):=x^{2}(\widehat{\phi})(x), x \in \mathbb{R}$. By the spectral theory (see for example [50]), for every $f \in L^{2}(X)$, we have the following Calderón reproducing formula

$$
f=c_{\psi} \lim _{\substack{\varepsilon \rightarrow 0+\\ N \rightarrow \infty}} \int_{\varepsilon}^{N} \psi(t \sqrt{L}) t^{2} L e^{-t \sqrt{L}} f \frac{d t}{t}
$$

with the limit converging in $L^{2}(X)$.

Fix $\delta \in(0,1 / 144)$ as in Proposition 2.1. There exists a system of dyadic cubes with parameter $\delta$ such that

$$
\mathscr{D}=\bigcup_{k \in \mathbb{Z}} \mathscr{D}_{k}, \quad \mathscr{D}_{k}=\left\{Q_{\beta}^{k}: \beta \in \mathscr{J}_{k}\right\} .
$$

For every $Q_{\beta}^{k} \in \mathscr{D}_{k}, k \in \mathbb{Z}, \beta \in \mathscr{J}_{k}$, define

$$
T\left(Q_{\beta}^{k}\right):=\left\{(x, t) \in X \times(0, \infty): x \in Q_{\beta}^{k}, \rho^{-1} \delta^{k+1} \leq t<\rho^{-1} \delta^{k}\right\},
$$

where $\rho>0$ is the parameter appeared in the support of $\phi$. We obtain a decomposition for $X \times(0, \infty)$ as follows:

$$
X \times(0, \infty)=\bigcup_{k \in \mathbb{Z}} \bigcup_{\beta \in \mathscr{J}_{k}} T\left(Q_{\beta}^{k}\right) .
$$

Then $T\left(Q_{\beta_{1}}^{k}\right) \cap T\left(Q_{\beta_{2}}^{\ell}\right)=\emptyset$ for arbitrary $Q_{\beta_{1}}^{k}, Q_{\beta_{2}}^{\ell} \in \mathscr{D}$ with $\beta_{1} \neq \beta_{2}$ or $k \neq \ell$. Therefore, for every $f \in L^{2}(X)$, we use A. Calderón trick ([6]) to write

$$
\begin{aligned}
f(x) & =c_{\psi} \iint_{X \times(0, \infty)} K_{\psi(t \sqrt{L})}(x, y)\left(t^{2} L e^{-t \sqrt{L}} f(y)\right) \frac{d \mu(y) d t}{t} \\
& =c_{\psi} \sum_{k \in \mathbb{Z}} \sum_{\beta \in \mathscr{F}_{k}} \iint_{T\left(Q_{\beta}^{k}\right)} K_{\psi(t \sqrt{L})}(x, y)\left(t^{2} L e^{-t \sqrt{L}} f(y)\right) \frac{d \mu(y) d t}{t} \\
& =: \sum_{k \in \mathbb{Z}} \sum_{\beta \in \mathscr{J}_{k}} a_{Q_{\beta}^{k}}(f)(x),
\end{aligned}
$$

where

$$
a_{Q_{\beta}^{k}}(f)(x)=c_{\psi} \iint_{T\left(Q_{\beta}^{k}\right)} K_{\psi(t \sqrt{L})}(x, y)\left(t^{2} L e^{-t \sqrt{L}} f(y)\right) \frac{d \mu(y) d t}{t} .
$$

Set

$$
\lambda_{Q_{\beta}^{k}}=\left(\iint_{T\left(Q_{\beta}^{k}\right)}\left|t^{2} L e^{-t \sqrt{L}} f(y)\right|^{2} \frac{d \mu(y) d t}{t}\right)^{1 / 2} .
$$

We have the following result.

Lemma 3.1. Suppose that $L$ is a densely-defined operator on $L^{2}(X)$ satisfying conditions $(\boldsymbol{H 1}),(\boldsymbol{H} 2)$ and $(\boldsymbol{H 3})$ with some $\theta \in(0,1]$. Then for every $k \in \mathbb{Z}$ and $\beta_{1} \in \mathscr{J}_{k}$, the function $a_{Q_{\beta_{1}}^{k}}(f)$ satisfies the following properties:

(i) $\operatorname{supp} a_{Q_{\beta_{1}}^{k}}(f) \subset 3 Q_{\beta_{1}}^{k} \subset B\left(x_{\beta_{1}}^{k}, 3 \delta^{k}\right) \subset E_{2}\left(Q_{\beta_{1}}^{k}\right)$;

(ii) $\left\|a_{Q_{\beta_{1}}^{k}}(f)\right\|_{\infty} \leq C \lambda_{Q_{\beta_{1}}^{k}} \mu\left(Q_{\beta_{1}}^{k}\right)^{-1 / 2}$; 
(iii) $\left|a_{Q_{\beta_{1}}^{k}}(f)(x)-a_{Q_{\beta_{1}}^{k}}(f)\left(x^{\prime}\right)\right| \leq C \lambda_{Q_{\beta_{1}}^{k}} \min \left\{\left(\frac{d\left(x, x^{\prime}\right)}{\delta^{k}}\right)^{\theta}, 1\right\} \mu\left(Q_{\beta_{1}}^{k}\right)^{-1 / 2}$ for all $x, x^{\prime} \in X$;

(iv) For every $\ell \leq k$, if $3 Q_{\beta_{1}}^{k} \cap 3 Q_{\beta_{2}}^{\ell} \neq \emptyset$, then

$$
\left|\int a_{Q_{\beta_{1}}^{k}}(f)(x) \overline{a_{Q_{\beta_{2}}^{\ell}}(f)(x)} d \mu(x)\right| \leq C \lambda_{Q_{\beta_{1}}^{k}} \lambda_{Q_{\beta_{2}}^{\ell}} \delta^{k-\ell}\left(\frac{\mu\left(Q_{\beta_{1}}^{k}\right)}{\mu\left(Q_{\beta_{2}}^{\ell}\right)}\right)^{1 / 2} .
$$

Proof. The result of (i) and (ii) is a straightforward application of (i) of Lemma 2.3.

For every $x, x^{\prime} \in X$,

$$
\left|a_{Q_{\beta_{1}}^{k}}(f)(x)-a_{Q_{\beta_{1}}^{k}}(f)\left(x^{\prime}\right)\right| \leq C \iint_{T\left(Q_{\beta_{1}}^{k}\right)}\left|K_{\psi(t \sqrt{L})}(x, y)-K_{\psi(t \sqrt{L})}\left(x^{\prime}, y\right)\right|\left|t^{2} L e^{-t \sqrt{L}} f(y)\right| \frac{d \mu(y) d t}{t} .
$$

From this, we apply (iii) of Lemma 2.3 and the Cauchy-Schwarz inequality to obtain (iii).

Let us prove (iv). For every $\ell \leq k$, we have that $\delta^{k} \leq \delta^{\ell}$. Note that $\psi(t \sqrt{L})$ is self-adjoint and so $K_{\psi(t \sqrt{L})}(x, y)=K_{\psi(t \sqrt{L})}(y, x)$. Thus

$$
\begin{aligned}
& \left|\int a_{Q_{\beta_{1}}^{k}}(f)(x) \overline{a_{Q_{\beta_{2}}^{\ell}}(f)(x)} d \mu(x)\right| \\
& \quad \leq C \iint_{T\left(Q_{\beta_{1}}^{k}\right)}\left|t^{2} L e^{-t \sqrt{L}} f(y)\right| \iint_{T\left(Q_{\beta_{2}}^{\ell}\right)}\left|K_{\psi(s \sqrt{L}) \psi(t \sqrt{L})}(y, z)\right|\left|s^{2} L e^{-s \sqrt{L}} f(z)\right| \frac{d \mu(z) d s}{s} \frac{d \mu(y) d t}{t} .
\end{aligned}
$$

By (ii) of Lemma 2.3, $\left|K_{\psi(s \sqrt{L}) \psi(t \sqrt{L})}(y, z)\right| \leq C t /(s \mu(B(y, s)))$. By the Cauchy-Schwarz inequality,

$$
\left|\int a_{Q_{\beta_{1}}^{k}}(f)(x) \overline{a_{Q_{\beta_{2}}^{\ell}}(f)(x)} d \mu(x)\right| \leq C \lambda_{Q_{\beta_{1}}^{k}} \lambda_{Q_{\beta_{2}}^{\ell}} \delta^{k-\ell}\left(\frac{\mu\left(Q_{\beta_{1}}^{k}\right)}{\mu\left(Q_{\beta_{2}}^{\ell}\right)}\right)^{1 / 2},
$$

which implies (iv). The proof of Lemma 3.1 is end.

Let us now introduce the discrete square function associated to an operator $L$. For a given $f \in$ $L^{2}(X)$, by (3.1) we have that $f=\sum_{Q \in \mathscr{D}} a_{Q}(f)$ where $a_{Q}(f)$ is given in (3.2). It follows from (3.1) and Proposition 2.2 that

$$
f(x)=\sum_{b=1}^{K} f_{b}(x), \text { where } f_{b}(x)=\sum_{Q \in \mathscr{D}, E_{2}(Q) \in \mathcal{D}^{b}} a_{Q}(f)(x) .
$$

Define the discrete square function associated to L:

$$
\mathscr{A}_{L}(f)(x):=\left(\sum_{Q \in \mathscr{D}} \frac{\left|\lambda_{Q}\right|^{2}}{\mu(Q)} \chi_{E_{2}(Q)}(x)\right)^{1 / 2},
$$

where $\lambda_{Q}$ is given in (3.3).

Lemma 3.2. Fix $\delta \in(0,1 / 144)$ as in Proposition 2.1 and let $\alpha>0$. Let $S_{L, \alpha} f$ and $\mathscr{A}_{L}(f)$ be given in (1.3) and (3.5), respectively. Then there exists $\rho=\rho(\alpha, \delta)$ in Lemma 2.3 such that

$$
c^{-1} S_{L, \delta \alpha / 75} f(x) \leq \mathscr{A}_{L}(f)(x) \leq c S_{L, \alpha} f(x) \text { a.e. } x \in X
$$

for some positive constant $c=c(n, \alpha)$.

As a consequence, we have

$$
\|f\|_{p} \simeq\left\|S_{L, \alpha} f\right\|_{p} \simeq\left\|\mathscr{A}_{L} f\right\|_{p}, \quad 1<p<\infty .
$$


Proof. Let $\rho=\delta^{2} \alpha / 5$. It follows from (3.5), (2.4) and the doubling property (2.1) that

$$
\mathscr{A}_{L}(f)(x) \simeq\left(\sum_{k \in \mathbb{Z}} \int_{\rho^{-1} \delta^{k+1}}^{\rho^{-1} \delta^{k}} \int_{\Omega_{k}(x)}\left|t^{2} L e^{-t \sqrt{L}} f(y)\right|^{2} \frac{d \mu(y)}{\mu(B(x, t))} \frac{d t}{t}\right)^{1 / 2},
$$

where $\Omega_{k}(x)=\bigcup_{Q \in \mathscr{D}_{k}, x \in E_{2}(Q)} Q$.

For any $y \in \Omega_{k}(x)$, there exists a unique $Q \in \mathscr{D}_{k}$ with $x \in E_{2}(Q)$ such that $y \in Q$. Denote the center point of $Q$ by $x_{Q}$, one can apply Proposition 2.2 and (2.10) to see $E_{2}(Q) \subset B\left(x_{Q}, 4 \delta^{k-1}\right)$, and so $d\left(x, x_{Q}\right) \leq 4 \delta^{k-1}$. Note that $Q \subset B\left(x_{Q}, 2 \delta^{k}\right)$. By (2.4), we have

$$
d(x, y) \leq d\left(x, x_{Q}\right)+d\left(y, x_{Q}\right) \leq 4 \delta^{k-1}+2 \delta^{k}<5 \delta^{k-1} .
$$

Recall that $\rho=\delta^{2} \alpha / 5$ so $\left[\rho^{-1} \delta^{k+1}, \rho^{-1} \delta^{k}\right)=\left[5 \delta^{k-1} / \alpha, 5 \delta^{k-2} / \alpha\right)$. Therefore, for every $(y, t) \in \Omega_{k}(x) \times$ $\left[\rho^{-1} \delta^{k+1}, \rho^{-1} \delta^{k}\right)$, we have $d(x, y)<5 \delta^{k-1} \leq \alpha t$. This, in combination with the definition of $S_{L, \alpha}(f)$, deduces that $\mathscr{A}_{L}(f)(x) \leq c S_{L, \alpha}(f)(x)$.

On the other hand, recall that $\delta<1 / 144$ and $\rho=\delta^{2} \alpha / 5$. For each $z \in B(x, \delta \alpha t / 75)$, there exists a unique $P \in \mathscr{D}_{k}$ with center point $x_{P}$ such that $z \in P$. Then for $t \in\left[5 \delta^{k-1} / \alpha, 5 \delta^{k-2} / \alpha\right)$, $d\left(x, x_{P}\right) \leq d\left(z, x_{P}\right)+d(x, z) \leq 2 \delta^{k}+\delta \alpha t / 75<\delta^{k-1} / 12$, which implies $x \in E_{2}(P), z \in \Omega_{k}(x)$ and so $B(x, \delta \alpha t / 75) \subset \Omega_{k}(x)$, hence $S_{L, \delta \alpha / 75}(f)(x) \leq c \mathscr{A}_{L}(f)(x)$. The proof of Lemma 3.2 is end.

\section{EXPONENTIAL-SQUARE INTEGRABILITY}

In this section we aim to establish the local exponential-square integrability of functions whose square functions associated to an operator $L$ are bounded, and extend a result of Chang-Wilson-Wolff [8, Theorem 3.2]. Based on Lemma 3.2, the proof is using reduction to the discrete square function associated to an operator $L$ on space $X$ of homogeneous type.

Throughout this section we fix a $Q \in \mathcal{D}^{b}$ for some $b \in\{1,2, \cdots, K\}$. Let $\mathcal{G} \subset \bigcup_{|k| \leq K_{\mathcal{G}}} \mathcal{D}_{k}^{b}$ be a subset such that $\bigcup_{P \in \mathscr{D}, E_{2}(P) \in \mathcal{G}} E_{2}(P) \subset Q$ and some $K_{\mathcal{G}}<\infty$. For every $f \in L^{2}(X)$, it follows from (3.1) that $f=\sum_{P \in \mathscr{D}} a_{P}(f)$. For such $\mathcal{G}$, we define

$$
f_{\mathcal{G}}=\sum_{P \in \mathscr{D}, E_{2}(P) \in \mathcal{G}} a_{P}(f)
$$

and

$$
\mathscr{A}_{L}\left(f_{\mathcal{G}}\right)(x)=\left(\sum_{P \in \mathscr{D}, E_{2}(P) \in \mathcal{G}} \frac{\left|\lambda_{P}\right|^{2}}{\mu(P)} \chi_{E_{2}(P)}(x)\right)^{1 / 2}
$$

where $\lambda_{P}$ is given in (3.3), and $E_{2}(P)$ is as in Proposition 2.2. Obviously, $\mathscr{A}_{L}\left(f_{\mathcal{G}}\right) \in L^{\infty}(X)$. Moreover, it can be verified that there exists a constant $C_{1}>0$, independent of $f_{\mathcal{G}}$ and $Q$ such that

$$
\left|f_{\mathcal{G}}\right|_{Q}^{2} \leq \frac{\left\|f_{\mathcal{G}}\right\|_{2}^{2}}{\mu(Q)} \leq C_{1}^{2}\left\|\mathscr{A}_{L}\left(f_{\mathcal{G}}\right)\right\|_{L^{\infty}(Q)}^{2}
$$

Indeed, we assume that $Q \in \mathcal{D}_{k_{0}}^{b}$ for some $k_{0} \in \mathbb{Z}$. Since $E_{2}(P) \subseteq Q$, there exists some $j \geq k_{0}$ such that $E_{2}(P) \in \mathcal{D}_{j}^{b}$. Note that $\operatorname{supp} a_{P}(f) \subset E_{2}(P)$. Thus we have

$$
\frac{\left\|f_{\mathcal{G}}\right\|_{2}^{2}}{\mu(Q)} \leq \frac{1}{\mu(Q)} \sum_{P \in \mathscr{D}, E_{2}(P) \in \mathcal{G}} \int_{Q}\left|a_{P}(f)(x)\right|^{2} d \mu(x)
$$




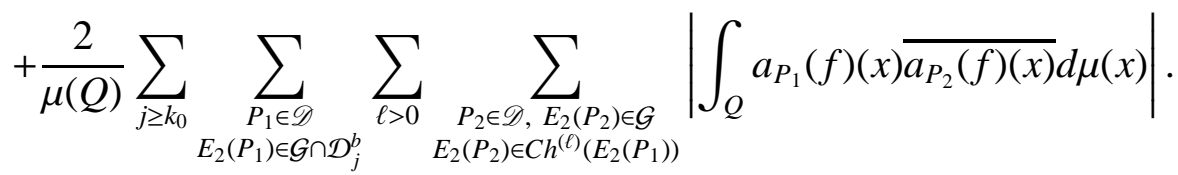

For $E_{2}\left(P_{1}\right) \in \mathcal{G} \cap \mathcal{D}_{j}^{b}$, and $E_{2}\left(P_{2}\right) \in C h^{(\ell)}\left(E_{2}\left(P_{1}\right)\right)$ mentioned above, we have $E_{2}\left(P_{2}\right) \in \mathcal{D}_{j+\ell}^{b}$, and the definition of $E_{2}$ implies that $P_{1} \in \mathscr{D}_{j+1}$ and $P_{2} \in \mathscr{D}_{j+\ell+1}$. Hence it follows from (iv) of Lemma 3.1 and Proposition 2.2 that there exists a constant $C=C(n, \rho)$ such that

$$
\left|\int_{Q} a_{P_{1}}(f)(x) \overline{a_{P_{2}}(f)(x)} d \mu(x)\right| \leq C \sqrt{\frac{\mu\left(E_{2}\left(P_{2}\right)\right)}{\mu\left(E_{2}\left(P_{1}\right)\right)}} \delta^{\ell}\left|\lambda_{P_{1}}\right|\left|\lambda_{P_{2}}\right| \leq C \delta^{\ell}\left(\left|\lambda_{P_{2}}\right|^{2}+\frac{\mu\left(E_{2}\left(P_{2}\right)\right)}{\mu\left(E_{2}\left(P_{1}\right)\right)}\left|\lambda_{P_{1}}\right|^{2}\right) .
$$

This, together with (ii) of Lemma 3.1, yields

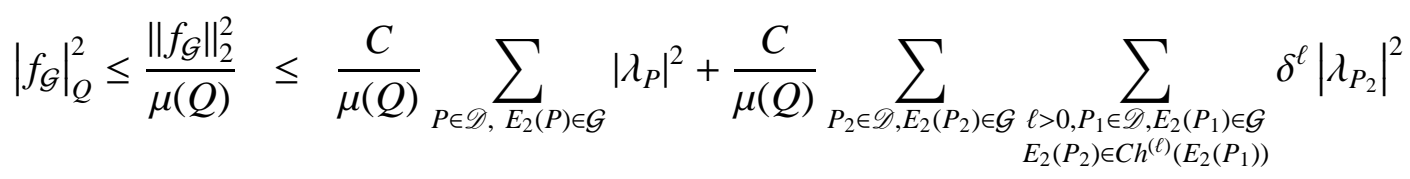

$$
\begin{aligned}
& +\frac{C}{\mu(Q)} \sum_{P_{1} \in \mathscr{D}, E_{2}\left(P_{1}\right) \in \mathcal{G}} \sum_{\ell>0} \delta^{\ell}\left|\lambda_{P_{1}}\right|^{2} \sum_{\substack{P_{2} \in \mathscr{D}, E_{2}\left(P_{2}\right) \in \mathcal{G} \\
E_{2}\left(P_{2}\right) \in C h^{(\ell)}\left(E_{2}\left(P_{1}\right)\right)}} \frac{\mu\left(E_{2}\left(P_{2}\right)\right)}{\mu\left(E_{2}\left(P_{1}\right)\right)} \\
& \leq \frac{C}{\mu(Q)} \sum_{P \in \mathscr{D}, E_{2}(P) \in \mathcal{G}}\left|\lambda_{P}\right|^{2} \\
& \leq \frac{C}{\mu(Q)} \int_{Q_{P \in \mathscr{D}, E_{2}(P) \in \mathcal{G}}} \frac{\left|\lambda_{P}\right|^{2}}{\mu(P)} \chi_{E_{2}(P)}(x) d \mu(x) \\
& \leq C_{1}^{2}\left\|\mathscr{A}_{L}\left(f_{\mathcal{G}}\right)\right\|_{L^{\infty}(Q)}^{2}
\end{aligned}
$$

for some $C_{1}>0$. This shows (4.3) and this estimate will be used often in the sequel.

4.1. John-Nirenberg type inequality for the discrete square function. In this subsection, we will give some preliminary result about a John-Nirenberg type inequality for the function $f_{\mathcal{G}}$.

Proposition 4.1. Suppose that $L$ is a densely-defined operator on $L^{2}(X)$ satisfying (H1), (H2) and (H3). Let $f \in L^{2}(X, d \mu)$. For a fixed $Q \in \mathcal{D}^{b}$ for some $b \in\{1,2, \cdots, K\}$, let $f_{\mathcal{G}}$ be given in (4.1). Then there exists two constants $c_{1}=c_{1}(n, \rho), C_{2}=C_{2}(n, \rho)$ independent of $\mathcal{G}, K_{\mathcal{G}}, Q$ and $f$, with $0<c_{1}<1<C_{2}<\infty$, such that for all $\lambda>0$,

$$
\mu\left(\left\{x \in Q:\left|f_{\mathcal{G}}(x)\right|>\lambda\right\}\right) \leq C_{2} \exp \left(-\frac{c_{1} \lambda}{\left\|\mathscr{A}_{L}\left(f_{\mathcal{G}}\right)\right\|_{L^{\infty}(Q)}}\right) \mu(Q) .
$$

As a consequence, we have

$$
\int_{Q} \exp \left(\frac{c_{1}\left|f_{\mathcal{G}}(x)\right|}{2\left\|\mathscr{A}_{L}\left(f_{\mathcal{G}}\right)\right\|_{L^{\infty}(Q)}}\right) d \mu(x) \leq C_{2} \mu(Q)
$$

Proof. One writes $E_{\lambda}=\left\{x \in Q:\left|f_{\mathcal{G}}(x)\right|>\lambda\right\}$ and $\lambda_{0}=\mathscr{N}\left\|\mathscr{A}_{L}\left(f_{\mathcal{G}}\right)\right\|_{L^{\infty}(Q)}$ for some constant $\mathscr{N}>C_{1}$, which will be chosen later. Let us consider two cases.

Case (1): $\lambda \geq \lambda_{0}$.

In this case, noting that by (4.3) that $\left|f_{\mathcal{G}}\right|_{Q} \leq \lambda$, we can construct a subset $\left\{Q_{\lambda, i}: i \in \mathbb{N}\right\}$ of dyadic cubes $Q_{\lambda, i} \in \mathcal{D}^{b}$ by selecting all the maximum dyadic cubes $Q_{\lambda, i} \subset Q$ in $\mathcal{D}^{b}$ such that: 1) $Q_{\lambda, i}$ 
satisfies $\left|f_{\mathcal{G}}\right|_{Q_{\lambda, i}}>\lambda$; 2) $\widetilde{Q}_{\lambda, i}$ satisfies $\left|f_{\mathcal{G}}\right|_{\widetilde{Q}_{\lambda, i}} \leq \lambda$, where $\widetilde{Q}_{\lambda, i}$ is the parent of $Q_{\lambda, i}$. Moreover, we have the following properties:

3) Up to a subset of measure zero,

$$
E_{\lambda} \subset \bigcup_{i} Q_{\lambda, i}=: \Omega_{\lambda}
$$

4) If $\lambda_{1} \geq \lambda_{2}$, then $\Omega_{\lambda_{1}} \subset \Omega_{\lambda_{2}}$.

Indeed, we note that for every $Q_{\lambda_{1}, i} \subset \Omega_{\lambda_{1}},\left|f_{\mathcal{G}}\right|_{Q_{\lambda_{1}, i}}>\lambda_{1} \geq \lambda_{2}$. There is some $Q_{\lambda_{2}, j}$ such that $Q_{\lambda_{1}, i} \subset Q_{\lambda_{2}, j} \subset \Omega_{\lambda_{2}}$, and so 4) follows. For 3), denote the maximal operator

$$
M^{\mathcal{D}^{b}}(f)(x):=\sup _{x \in P \in \mathcal{D}^{b}} \frac{1}{\mu(P)} \int_{P}|f(y)| d \mu(y) .
$$

It follows from [33, Theorem 15.1] that $M^{\mathcal{D}^{b}}$ is of weak type $(1,1)$ uniformly in $b \in\{1,2, \cdots, K\}$. Therefore, a standard argument deduces the corresponding Lebesgue differentiation theorem

$$
\lim _{\substack{k \rightarrow+\infty \\ x \in P \in \mathcal{D}_{k}^{b}}} \frac{1}{\mu(P)} \int_{P} f(y) d \mu(y)=f(x) \text { a.e. } x \in X .
$$

This shows $E_{\lambda} \subset\left\{x \in Q: M^{\mathcal{D}^{b}}\left(f_{\mathcal{G}}\right)(x)>\lambda\right\} \subset \bigcup_{i} Q_{\lambda, i}$ up to a subset of measure zero. This finishes the proof of 3 ) above.

Now, let us fix $i$. To estimate $\left|\left(f_{\mathcal{G}}\right)_{Q_{\lambda, i}}\right|$, for $x \in Q_{\lambda, i}$ we rewrite $f_{\mathcal{G}}$ by

$$
f_{\mathcal{G}}(x)=\sum_{\substack{P \in \mathscr{D}, E_{2}(P) \in \mathcal{G} \\ \bar{Q}_{\lambda, i} \subset E_{2}(P)}} a_{P}(f)(x)+\sum_{\substack{P \in \mathscr{D}, E_{2}(P) \in \mathcal{G} \\ E_{2}(P) \subset Q_{\lambda, i}}} a_{P}(f)(x)=: f_{\mathcal{G}, 1}(x)+f_{\mathcal{G}, 2}(x) .
$$

Since $\mathcal{G} \subset \bigcup_{|k| \leq K_{\mathcal{G}}} \mathcal{D}_{k}^{b}$ with $K_{\mathcal{G}}<\infty$, (iii) of Lemma 3.1 implies that $f_{\mathcal{G}, 1}$ is a finite sum of continuous functions and so is continuous on $Q$. Therefore, for every $Q_{\lambda, i}$ there exists a point $x_{i} \in Q_{\lambda, i}$ such that

$$
\left(f_{\mathcal{G}, 1}\right)_{Q_{\lambda, i}}=f_{\mathcal{G}, 1}\left(x_{i}\right) .
$$

Similarly, there exists a point $\tilde{x}_{i} \in \widetilde{Q}_{\lambda, i}$ such that $\left(f_{\mathcal{G}, 1}\right)_{\widetilde{Q}_{\lambda, i}}=f_{\mathcal{G}, 1}\left(\tilde{x}_{i}\right)$. Denote that $\widetilde{Q}_{\lambda, i} \in \mathcal{D}_{\ell_{0}}^{b}$ and $E_{2}(P) \in \mathcal{D}_{\ell_{P}}^{b}$. Then one can apply (iii) of Lemma 3.1, Proposition 2.2 and Hölder's inequality to obtain

$$
\begin{aligned}
\left|f_{\mathcal{G}, 1}\left(x_{i}\right)-f_{\mathcal{G}, 1}\left(\tilde{x}_{i}\right)\right| \leq & \sum_{\substack{P \in \mathscr{D}, E_{2}(P) \in \mathcal{G} \\
\widetilde{Q}_{\lambda, i} \subset E_{2}(P)}}\left|a_{P}(f)\left(x_{i}\right)-a_{P}(f)\left(\tilde{x}_{i}\right)\right| \\
\leq & C \sum_{\substack{P \in \mathscr{D}, E_{2}(P) \in \mathcal{G} \\
\widetilde{Q}_{\lambda, i} \subset E_{2}(P)}}\left(\frac{d\left(x_{i}, \tilde{x}_{i}\right)}{\delta^{\ell_{P}+1}}\right)^{\theta} \frac{\left|\lambda_{P}\right|}{\mu(P)^{1 / 2}} \\
\leq & C \sum_{\substack{P \in \mathscr{D}, E_{2}(P) \in \mathcal{G} \\
\widetilde{Q}_{\lambda, i} \subset E_{2}(P)}} \delta^{-\theta}\left(\frac{\delta^{\ell_{0}}}{\delta^{\ell_{P}}}\right)^{\theta} \frac{\left|\lambda_{P}\right|}{\mu(P)^{1 / 2}} \\
\leq & \left.C \delta^{-\theta} \sum_{\substack{P \in \mathscr{D}, E_{2}(P) \in \mathcal{G} \\
\widetilde{Q}_{\lambda, i} \subset E_{2}(P)}}\left(\delta^{\ell_{0}-\ell_{P}}\right)^{2 \theta}\right)^{1 / 2}\left(\sum_{\substack{P \in \mathscr{D}, E_{2}(P) \in \mathcal{G} \\
\widetilde{Q}_{\lambda, i} E_{2}(P)}} \frac{\left|\lambda_{P}\right|^{2}}{\mu(P)}\right)^{1 / 2}
\end{aligned}
$$




$$
\leq \frac{C}{\delta^{\theta} \sqrt{1-\delta^{2 \theta}}}\left(\sum_{\substack{P \in \mathscr{D}, E_{2}(P) \in \mathcal{G} \\ \widetilde{Q}_{\lambda, i} \subset E_{2}(P)}} \frac{\left|\lambda_{P}\right|^{2}}{\mu(P)}\right)^{1 / 2},
$$

where constants $C$ above are dependent on $n$ and $\rho$. Note that $\widetilde{Q}_{\lambda, i} \subset E_{2}(P)$ implies $\chi_{E_{2}(P)}\left(x_{i}\right) \equiv 1$. Then we have

$$
\begin{aligned}
\left|f_{\mathcal{G}, 1}\left(x_{i}\right)-f_{\mathcal{G}, 1}\left(\tilde{x}_{i}\right)\right| & \leq \frac{C}{\delta^{\theta} \sqrt{1-\delta^{2 \theta}}}\left(\sum_{P \in \mathscr{D}, E_{2}(P) \in \mathcal{G}} \frac{\left|\lambda_{P}\right|^{2}}{\mu(P)} \chi_{E_{2}(P)}\left(x_{i}\right)\right)^{1 / 2} \\
& \leq C_{3}\left\|\mathscr{A}_{L}\left(f_{\mathcal{G}}\right)\right\|_{L^{\infty}(Q)}
\end{aligned}
$$

for some $C_{3}=\frac{C}{\delta^{\theta} \sqrt{1-\delta^{2 \theta}}}$.

Note that $\operatorname{supp} f_{\mathcal{G}, 2} \subset Q_{\lambda, i}$. Thus

$$
\left|\left(f_{\mathcal{G}, 2}\right)_{Q_{\lambda, i}}-\left(f_{\mathcal{G}, 2}\right)_{\widetilde{Q}_{\lambda, i}}\right|=\left|\frac{1}{\mu\left(Q_{\lambda, i}\right)}-\frac{1}{\mu\left(\widetilde{Q}_{\lambda, i}\right)}\right|\left|\int_{Q_{\lambda, i}} f_{\mathcal{G}, 2}(x) d \mu\right| \leq\left|f_{\mathcal{G}, 2}\right|_{Q_{\lambda, i}} .
$$

Taking $Q_{\lambda, i}$ as $Q$ and $f_{\mathcal{G}, 2}$ as $f_{\mathcal{G}}$ in (4.4), we have

$$
\left|\left(f_{\mathcal{G}, 2}\right)_{Q_{\lambda, i}}-\left(f_{\mathcal{G}, 2}\right)_{\widetilde{Q}_{\lambda, i}}\right| \leq\left|f_{\mathcal{G}, 2}\right|_{Q_{\lambda, i}} \leq C_{1}\left\|\mathscr{A}_{L}\left(f_{\mathcal{G}, 2}\right)\right\|_{L^{\infty}\left(Q_{\lambda, i}\right)} \leq C_{1}\left\|\mathscr{A}_{L}\left(f_{\mathcal{G}}\right)\right\|_{L^{\infty}(Q)},
$$

where $C_{1}$ is the constant in (4.4).

Therefore, when $\lambda \geq \lambda_{0}$,

$$
\begin{aligned}
\left|\left(f_{\mathcal{G}}\right)_{Q_{\lambda, i}}\right| & \leq\left|\left(f_{\mathcal{G}}\right)_{Q_{\lambda, i}}-\left(f_{\mathcal{G}}\right)_{\widetilde{Q}_{\lambda, i}}\right|+\left|\left(f_{\mathcal{G}}\right)_{\widetilde{Q}_{\lambda, i}}\right| \\
& \leq\left|\left(f_{\mathcal{G}, 1}\right)_{Q_{\lambda, i}}-\left(f_{\mathcal{G}, 1}\right)_{\widetilde{Q}_{\lambda, i}}\right|+\left|\left(f_{\mathcal{G}, 2}\right)_{Q_{\lambda, i}}-\left(f_{\mathcal{G}, 2}\right)_{\widetilde{Q}_{\lambda, i}}\right|+\left|\left(f_{\mathcal{G}}\right)_{\widetilde{Q}_{\lambda, i}}\right| \\
& \leq C_{4}\left\|\mathscr{A}_{L}\left(f_{\mathcal{G}}\right)\right\|_{L^{\infty}(Q)}+\lambda
\end{aligned}
$$

with $C_{4}=C_{1}+C_{3}$.

Now, take a parameter $\lambda^{\prime}>0$ which will be chosen later. Similarly, let $\left\{Q_{\lambda+\lambda^{\prime}, j}\right\}, Q_{\lambda+\lambda^{\prime}, j} \in \mathcal{D}^{b}$, be the maximal dyadic cubes such that $\left|f_{\mathcal{G}}\right|_{Q_{\lambda+\lambda^{\prime}, j}}>\lambda+\lambda^{\prime}$. Then for all $Q_{\lambda+\lambda^{\prime}, j}$, we have $\mu\left(Q_{\lambda+\lambda^{\prime}, j}\right) \leq$ $\frac{1}{\lambda+\lambda^{\prime}} \int_{Q_{\lambda+\lambda^{\prime}, j}}\left|f_{\mathcal{G}}\right| d \mu(x)$. From the selection rule of $Q_{\lambda+\lambda^{\prime}, j}$ and $Q_{\lambda, i}$, we know that if $Q_{\lambda+\lambda^{\prime}, j} \cap Q_{\lambda, i} \neq \emptyset$ then $Q_{\lambda+\lambda^{\prime}, j} \subset Q_{\lambda, i}$. For every fixed $Q_{\lambda, i}$, by (4.9)

$$
\begin{aligned}
\mu\left(\Omega_{\lambda+\lambda^{\prime}} \cap Q_{\lambda, i}\right) \leq & \frac{1}{\lambda+\lambda^{\prime}} \sum_{j: Q_{\lambda+\lambda^{\prime}, j} \subset Q_{\lambda, i}} \int_{Q_{\lambda+\lambda^{\prime}, j}}\left|f_{\mathcal{G}}\right| d \mu(x) \\
\leq & \frac{1}{\lambda+\lambda^{\prime}} \int_{Q_{\lambda, i}}\left|f_{\mathcal{G}}-\left(f_{\mathcal{G}}\right)_{Q_{\lambda, i}}\right| d \mu(x)+\frac{\left|\left(f_{\mathcal{G}}\right)_{Q_{\lambda, i}}\right|}{\lambda+\lambda^{\prime}} \mu\left(\Omega_{\lambda+\lambda^{\prime}} \cap Q_{\lambda, i}\right) \\
\leq & \frac{1}{\lambda+\lambda^{\prime}} \int_{Q_{\lambda, i}}\left|f_{\mathcal{G}, 1}-\left(f_{\mathcal{G}, 1}\right)_{Q_{\lambda, i}}\right| d \mu(x)+\frac{2}{\lambda+\lambda^{\prime}}\left|f_{\mathcal{G}, 2}\right|_{Q_{\lambda, i}} \mu\left(Q_{\lambda, i}\right) \\
& +\frac{C_{4}\left\|\mathscr{A}_{L}\left(f_{\mathcal{G}}\right)\right\|_{L^{\infty}(Q)}+\lambda}{\lambda+\lambda^{\prime}} \mu\left(\Omega_{\lambda+\lambda^{\prime}} \cap Q_{\lambda, i}\right) .
\end{aligned}
$$

For $x \in Q_{\lambda, i}$, we apply the argument in (4.7) and (4.8) to obtain

$$
\left|f_{\mathcal{G}, 1}(x)-\left(f_{\mathcal{G}, 1}\right)_{Q_{\lambda, i}}\right| \leq C_{3}\left\|\mathscr{A}_{L}\left(f_{\mathcal{G}}\right)\right\|_{L^{\infty}(Q)}
$$


and

$$
\left|f_{\mathcal{G}, 2}\right|_{Q_{\lambda, i}} \leq C_{1}\left\|\mathscr{A}_{L}\left(f_{\mathcal{G}}\right)\right\|_{L^{\infty}(Q)}
$$

Putting (4.11) and (4.12) into (4.10), we have

$$
\mu\left(\Omega_{\lambda+\lambda^{\prime}} \cap Q_{\lambda, i}\right) \leq \frac{C_{5}\left\|\mathscr{A}_{L}\left(f_{\mathcal{G}}\right)\right\|_{L^{\infty}(Q)}}{\lambda+\lambda^{\prime}} \mu\left(Q_{\lambda, i}\right)+\frac{C_{4}\left\|\mathscr{A}_{L}\left(f_{\mathcal{G}}\right)\right\|_{L^{\infty}(Q)}+\lambda}{\lambda+\lambda^{\prime}} \mu\left(\Omega_{\lambda+\lambda^{\prime}} \cap Q_{\lambda, i}\right)
$$

with $C_{5}=2 C_{1}+C_{3}$. Therefore,

$$
\mu\left(\Omega_{\lambda+\lambda^{\prime}} \cap Q_{\lambda, i}\right) \leq \frac{C_{5}\left\|\mathscr{A}_{L}\left(f_{\mathcal{G}}\right)\right\|_{L^{\infty}(Q)}}{\lambda^{\prime}-C_{4}\left\|\mathscr{A}_{L}\left(f_{\mathcal{G}}\right)\right\|_{L^{\infty}(Q)}} \mu\left(Q_{\lambda, i}\right) .
$$

This tells us that for every $\lambda \geq \lambda_{0}$,

$$
\mu\left(\Omega_{\lambda+\lambda^{\prime}}\right) \leq \frac{C_{5}\left\|\mathscr{A}_{L}\left(f_{\mathcal{G}}\right)\right\|_{L^{\infty}(Q)}}{\lambda^{\prime}-C_{4}\left\|\mathscr{A}_{L}\left(f_{\mathcal{G}}\right)\right\|_{L^{\infty}(Q)}} \mu\left(\Omega_{\lambda}\right) .
$$

Let $\lambda^{\prime}=\lambda_{0}$. For $\lambda \geq \lambda_{0}$, there exists a unique $k \geq 1$ such that $k \lambda_{0} \leq \lambda<(k+1) \lambda_{0}$. Therefore,

$$
\begin{aligned}
\mu\left(E_{\lambda}\right) & \leq \mu\left(\Omega_{\lambda}\right) \leq \mu\left(\Omega_{k \lambda_{0}}\right) \\
& \leq \frac{C_{5}\left\|\mathscr{A}_{L}\left(f_{\mathcal{G}}\right)\right\|_{L^{\infty}(Q)}}{\lambda_{0}-C_{4}\left\|\mathscr{A}_{L}\left(f_{\mathcal{G}}\right)\right\|_{L^{\infty}(Q)}} \mu\left(\Omega_{(k-1) \lambda_{0}}\right) .
\end{aligned}
$$

Now choose the constant $\mathscr{N}=4 C_{4}$, and then $\lambda_{0}=4 C_{4}\left\|\mathscr{A}_{L}\left(f_{\mathcal{G}}\right)\right\|_{L^{\infty}(Q)}$. Iterating $k-1$ times, we notice that $k-1>\lambda / \lambda_{0}-2$,

$$
\begin{aligned}
\mu\left(E_{\lambda}\right) & \leq \exp \left\{-(k-1) \log \left(\frac{\lambda_{0}-C_{4}\left\|_{\mathscr{A}_{L}}\left(f_{\mathcal{G}}\right)\right\|_{L^{\infty}(Q)}}{C_{5}\left\|\mathscr{A}_{L}\left(f_{\mathcal{G}}\right)\right\|_{L^{\infty}(Q)}}\right)\right\} \mu\left(\Omega_{\lambda_{0}}\right) \\
& \leq C_{2} \exp \left(-\frac{c_{1} \lambda}{\left\|\mathscr{A}_{L}\left(f_{\mathcal{G}}\right)\right\|_{L^{\infty}(Q)}}\right) \mu(Q),
\end{aligned}
$$

where

$$
c_{1}=\frac{1}{4 C_{4}} \log \left(\frac{3 C_{4}}{C_{5}}\right), \quad C_{2}=\left(\frac{3 C_{4}}{C_{5}}\right)^{2}=\exp \left(8 C_{4} c_{1}\right)
$$

Case (2): $\lambda<\lambda_{0}$.

In this case, we write

$$
\begin{aligned}
\mu\left(E_{\lambda}\right) & <\exp \left(\frac{c_{1} \lambda_{0}}{\left\|\mathscr{A}_{L}\left(f_{\mathcal{G}}\right)\right\|_{L^{\infty}(Q)}}\right) \exp \left(-\frac{c_{1} \lambda}{\left\|\mathscr{A}_{L}\left(f_{\mathcal{G}}\right)\right\|_{L^{\infty}(Q)}}\right) \mu(Q) \\
& =\sqrt{C_{2}} \exp \left(-\frac{c_{1} \lambda}{\left\|\mathscr{A}_{L}\left(f_{\mathcal{G}}\right)\right\|_{L^{\infty}(Q)}}\right) \mu(Q),
\end{aligned}
$$

which, in combination with estimate (4.13) of Case (1), yields (4.5).

Estimate (4.6) is a consequence of (4.5). The proof of Proposition 4.1 is complete. 
4.2. Exponential-square estimates. To prove our main Theorem 4.4 below, we first show that the boundedness of the discrete square function implies its local exponential-square integrability. Recall that $c_{1}$ and $C_{2}$ are two constants given in (4.5). Then we have the following result.

Theorem 4.2. Suppose that $L$ is a densely-defined operator on $L^{2}(X)$ satisfying $(\boldsymbol{H 1}),(\boldsymbol{H} 2)$ and (H3). Let $f \in L^{2}(X, d \mu)$. For a fixed $Q \in \mathcal{D}^{b}$ for some $b \in\{1,2, \cdots, K\}$, and $f_{\mathcal{G}}$ be given in (4.1). Then there exists a constant $c_{2}=c_{2}(n, \rho) \in(0,1)$ independent of $\mathcal{G}, K_{\mathcal{G}}, Q$ and $f$ such that for all $\lambda>0$,

$$
\mu\left(\left\{x \in Q:\left|f_{\mathcal{G}}(x)\right|>\lambda\right\}\right) \leq C_{2} \exp \left(-c_{2} \frac{\lambda^{2}}{\left\|\mathscr{A}_{L}\left(f_{\mathcal{G}}\right)\right\|_{L^{\infty}(Q)}^{2}}\right) \mu(Q) .
$$

As a consequence, there exist two positive constants $c_{3}=c_{3}(n, \rho) \in\left(0, c_{2}\right)$ and $C_{6}=C_{6}(n, \rho)$ independent of $\mathcal{G}, K_{\mathcal{G}}, Q$ and $f$ such that

$$
\frac{1}{\mu(Q)} \int_{Q} \exp \left(\frac{c_{3}\left|f_{\mathcal{G}}(x)\right|^{2}}{\left\|\mathscr{A}_{L}\left(f_{\mathcal{G}}\right)\right\|_{L^{\infty}(Q)}^{2}}\right) d \mu(x) \leq C_{6} .
$$

Proof. To prove (4.14), it is enough to show that for $c_{4}=c_{1} / 4$, there exists a positive constant $C_{7}=C_{7}(n, \rho)$ independent of $f$ such that

$$
\int_{Q} \exp \left(c_{4}\left(\left|f_{\mathcal{G}}\right|-C_{7}\left\|\mathscr{A}_{L}\left(f_{\mathcal{G}}\right)\right\|_{L^{\infty}(Q)}^{2}\right)\right) d \mu(x) \leq C_{2} \mu(Q) .
$$

Once (4.16) is proven, (4.14) follows readily. Indeed, we take $g=\lambda f /\left[2 C_{7}\left\|\mathscr{A}_{L}\left(f_{\mathcal{G}}\right)\right\|_{L^{\infty}(Q)}^{2}\right] \in L^{2}(X)$. Replacing $f_{\mathcal{G}}$ by $g_{\mathcal{G}}$ in (4.16) and using the fact $\left|(C f)_{\mathcal{G}}\right|=C\left|f_{\mathcal{G}}\right|$ and $\mathscr{A}_{L}\left((C f)_{\mathcal{G}}\right)=C \mathscr{A}_{L}\left(f_{\mathcal{G}}\right)$ for any constant $C>0$, we have

$$
\int_{Q} \exp \left\{c_{4}\left(\frac{\lambda}{2 C_{7}\left\|\mathscr{A}_{L}\left(f_{\mathcal{G}}\right)\right\|_{L^{\infty}(Q)}^{2}}\left(\left|f_{\mathcal{G}}\right|-\frac{\lambda}{2}\right)\right)\right\} d \mu(x) \leq C_{2} \mu(Q) .
$$

From this, it is easy to see that (4.14) holds for $c_{2}=c_{4} /\left(4 C_{7}\right)$.

We now turn to verify (4.16). Observe that if $\left\|\mathscr{A}_{L}\left(f_{\mathcal{G}}\right)\right\|_{L^{\infty}(Q)} \leq 2$, we then apply (4.6) to obtain

$$
\int_{Q} \exp \left(c_{4}\left|f_{\mathcal{G}}\right|\right) d \mu(x) \leq C_{2} \mu(Q) .
$$

Then it reduces to show (4.16) for the case $\left\|\mathscr{A}_{L}\left(f_{\mathcal{G}}\right)\right\|_{L^{\infty}(Q)}>2$. Define $\mathcal{G}_{1}=\left\{E_{2}(P) \in \mathcal{G}\right.$ : $\left.\left|\lambda_{P}\right|^{2} / \mu(P)<1\right\}$ and $\mathcal{G}_{2}=\mathcal{G} \backslash \mathcal{G}_{1}$. We decompose $f_{\mathcal{G}}$ into two parts:

$$
f_{\mathcal{G}}=\sum_{E_{2}(P) \in \mathcal{G}_{1}} a_{P}(f)+\sum_{E_{2}(P) \in \mathcal{G}_{2}} a_{P}(f)=: f_{\mathcal{G}_{1}}+f_{\mathcal{G}_{2}} \text {. }
$$

Notice that $\left\|a_{P}(f)\right\|_{L^{\infty}(Q)} \leq C\left|\lambda_{P}\right| / \mu(P)^{1 / 2} \leq C\left|\lambda_{P}\right|^{2} / \mu(P)$ for $E_{2}(P) \in \mathcal{G}_{2}$, where $C$ is in (ii) of Lemma 3.1 and $0<C \leq C_{1}$ and $C_{1}$ is the constant in (4.4). This tells us that

$$
\left|f_{\mathcal{G}_{2}}(x)\right| \leq C_{1} \sum_{E_{2}(P) \in \mathcal{G}_{2}} \frac{\left|\lambda_{P}\right|^{2}}{\mu(P)} \chi_{E_{2}(P)}(x) .
$$

From this, we know

$$
\left|f_{\mathcal{G}}(x)\right|-C_{1} \mathscr{A}_{L}^{2}\left(f_{\mathcal{G}}\right)(x) \leq\left|f_{\mathcal{G}_{1}}(x)\right| .
$$

To continue, we consider the following two cases.

Case (1): $\left\|\mathscr{A}_{L}\left(f_{\mathcal{G}_{1}}\right)\right\|_{L^{\infty}(Q)} \leq 2$. 
In this case, we apply (4.17) for $f_{\mathcal{G}_{1}}$ and (4.18) to obtain

$$
\begin{aligned}
\int_{Q} \exp \left\{c_{4}\left(\left|f_{\mathcal{G}}(x)\right|-C_{1}\left\|\mathscr{A}_{L}\left(f_{\mathcal{G}}\right)\right\|_{L^{\infty}(Q)}^{2}\right)\right\} d \mu(x) & \leq \int_{Q} \exp \left\{c_{4}\left(\left|f_{\mathcal{G}}(x)\right|-C_{1} \mathscr{A}_{L}^{2}\left(f_{\mathcal{G}}\right)(x)\right)\right\} d \mu(x) \\
& \leq \int_{Q} \exp \left(c_{4}\left|f_{\mathcal{G}_{1}}(x)\right|\right) d \mu(x) \\
& \leq C_{2} \mu(Q) .
\end{aligned}
$$

Case (2): $\left\|\mathscr{A}_{L}\left(f_{\left.\mathcal{G}_{1}\right)}\right)\right\|_{L^{\infty}(Q)}>2$.

In this case, we assume $Q \in \mathcal{D}_{k_{0}}^{b}$ for some $k_{0} \leq K_{\mathcal{G}}$. Let

$$
\left\{R_{1}, R_{2}, \cdots, R_{M_{0}}\right\}=C h^{\left(K_{\mathcal{G}}-k_{0}\right)}(Q) \text {. }
$$

Then it follows that for every $R_{j}, 1 \leq j \leq M_{0}$, and $E_{2}(P) \in \mathcal{G}$, either $R_{j} \cap E_{2}(P)=\emptyset$ or $R_{j} \in$ $C h^{(\ell)}\left(E_{2}(P)\right)$ for some $\ell \geq 0$. For every $j=1,2, \cdots, M_{0}$, set

$$
\mathscr{L}_{j}=\left\{E_{2}(P) \in \mathcal{G}_{1}: R_{j} \subset E_{2}(P)\right\} .
$$

Let us consider $\mathscr{L}_{1}$. Note that when $\left\|\mathscr{A}_{L}\left(f_{\mathcal{G}_{1}}\right)\right\|_{L^{\infty}\left(R_{1}\right)}=0$, there does not exist $E_{2}(P) \in \mathcal{G}_{1}$ such that $R_{1} \in C h^{(\ell)}\left(E_{2}(P)\right)$ for some $\ell \geq 0$; when $\left\|\mathscr{A}_{L}\left(f_{\mathcal{G}_{1}}\right)\right\|_{L^{\infty}\left(R_{1}\right)} \neq 0$, there are a constant $K_{1}$ with $1 \leq K_{1} \leq \min \left\{2 K_{\mathcal{G}}+1, K_{\mathcal{G}}-k_{0}+1\right\}$ and a chain $\mathscr{L}_{1}$ :

$$
\mathscr{L}_{1}: \quad E_{2}\left(P_{1}\right) \supset E_{2}\left(P_{2}\right) \supset E_{2}\left(P_{3}\right) \supset \cdots \supset E_{2}\left(P_{K_{1}}\right), \quad E_{2}\left(P_{k}\right) \in \mathcal{G}_{1}, R_{1} \subset E_{2}\left(P_{k}\right), 1 \leq k \leq K_{1} .
$$

In the chain $\mathscr{L}_{1}$, if there exist $E_{2}\left(P_{k}\right)$ and $E_{2}\left(P_{k+1}\right)$ with $E_{2}\left(P_{k}\right)=E_{2}\left(P_{k+1}\right)$, then $E_{2}\left(P_{k+1}\right) \in$ $C h^{(\ell)} E_{2}\left(P_{k}\right)$ for some $\ell \geq 1$. Note that $E_{2}(P) \in \mathcal{G}_{1}$ means that $\left|\lambda_{P}\right|^{2} / \mu(P)<1$. We use the following method to classify cubes in the chain $\mathscr{L}_{1}$ in the following way:

(i): If

$$
\left(\sum_{k=1}^{K_{1}} \frac{\left|\lambda_{P_{k}}\right|^{2}}{\mu\left(P_{k}\right)}\right)^{1 / 2} \leq 2
$$

set $\operatorname{flag}\left(E_{2}\left(P_{k}\right)\right)=1$ for $1 \leq k \leq K_{1}$;

(ii): Otherwise, there exists a sequence of $N_{1}+1$ natural numbers $0=c_{0}<2 \leq c_{1}<c_{2}<\cdots<$ $c_{N_{1}} \leq K_{1}-1$ such that

$$
1<\left(\sum_{k=c_{i}+1}^{c_{i+1}} \frac{\left|\lambda_{P_{k}}\right|^{2}}{\mu\left(P_{k}\right)}\right)^{1 / 2} \leq 2, \quad\left(\sum_{k=c_{i}+1}^{c_{i+1}+1} \frac{\left|\lambda_{P_{k}}\right|^{2}}{\mu\left(P_{k}\right)}\right)^{1 / 2}>2, \quad i=0,1, \cdots, N_{1}-1,
$$

and

$$
\left(\sum_{k=c_{N_{1}}+1}^{K_{1}} \frac{\left|\lambda_{P_{k}}\right|^{2}}{\mu\left(P_{k}\right)}\right)^{1 / 2} \leq 2
$$

For every $i$ with $0 \leq i \leq N_{1}-1$, set flag $\left(E_{2}\left(P_{k}\right)\right)=i+1$ if $c_{i}+1 \leq k \leq c_{i+1}$. When $k \in\left[c_{N_{1}}+1, K_{1}\right]$, set flag $\left(E_{2}\left(P_{k}\right)\right)=N_{1}+1$.

To understand the above algorithm, we take an example of the case $K_{1}=8, c_{1}=3, c_{2}=6$ and $N_{1}=2$ to classify cubes in the chain $\mathscr{L}_{1}$ as follows. 


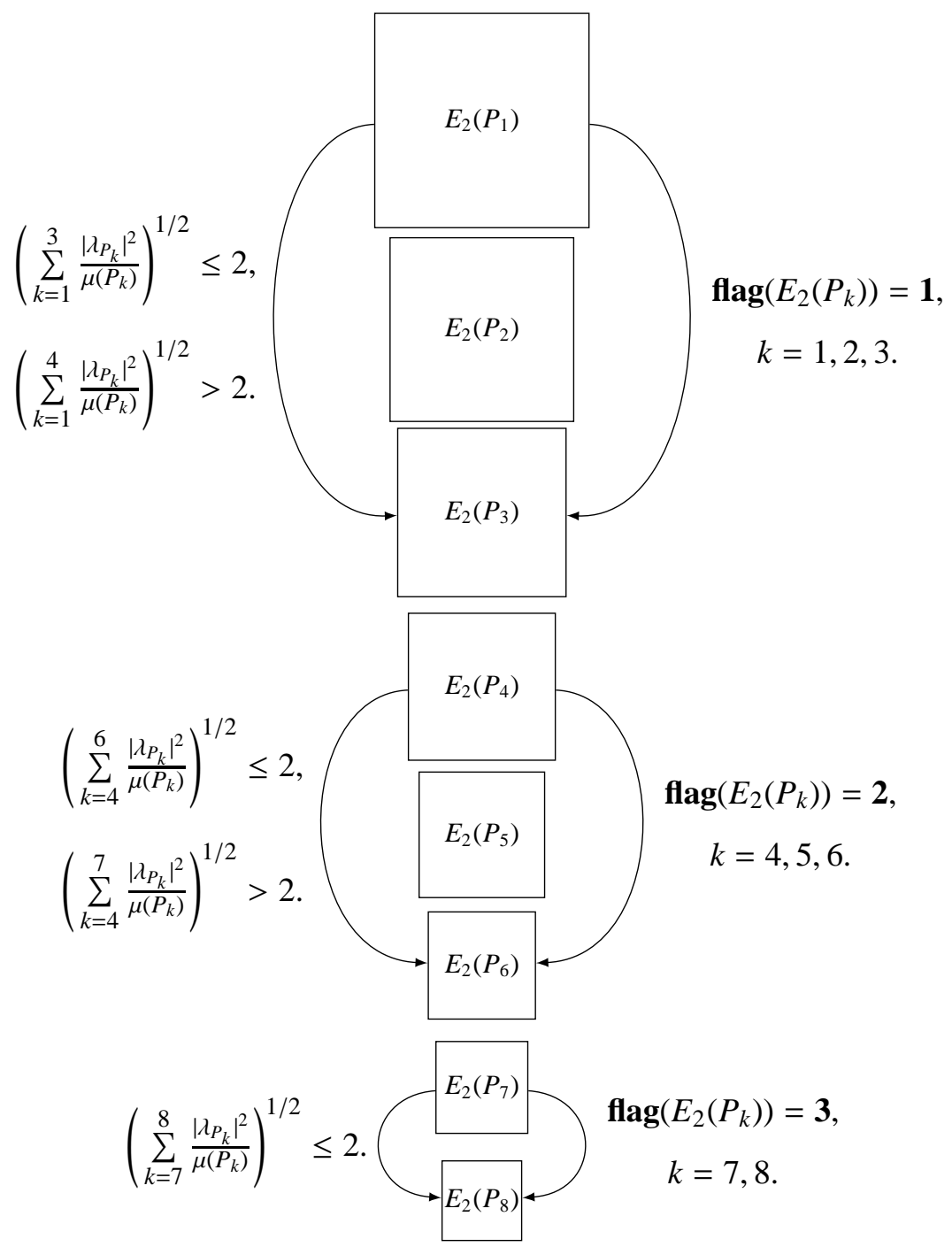

Figure 1. The case $K_{1}=8, c_{1}=3, c_{2}=6$ and $N_{1}=2$.

Now we estimate $N_{1}$. Note that for each $0 \leq i \leq N_{1}-1$

$$
1<\sum_{k=c_{i}+1}^{c_{i+1}} \frac{\left|\lambda_{P_{k}}\right|^{2}}{\mu\left(P_{k}\right)} \leq 4
$$

which gives

$$
N_{1}<\sum_{i=0}^{N_{1}-1} \sum_{k=c_{i}+1}^{c_{i+1}} \frac{\left|\lambda_{P_{k}}\right|^{2}}{\mu\left(P_{k}\right)}=\sum_{k=1}^{c_{N_{1}}} \frac{\left|\lambda_{P_{k}}\right|^{2}}{\mu\left(P_{k}\right)} \chi_{E_{2}(P)}\left(x_{0}\right) \leq \sum_{k=1}^{K_{1}} \frac{\left|\lambda_{P_{k}}\right|^{2}}{\mu\left(P_{k}\right)} \chi_{E_{2}(P)}\left(x_{0}\right) \leq 4 N_{1}+4
$$

for some $x_{0} \in R_{1}$, and so

Then we have

$$
N_{1}<\left\|\mathscr{A}_{L}\left(f_{\mathcal{G}_{1}}\right)\right\|_{L^{\infty}\left(R_{1}\right)}^{2} \leq\left\|\mathscr{A}_{L}\left(f_{\mathcal{G}_{1}}\right)\right\|_{L^{\infty}(Q)}^{2}
$$

$$
\max \left\{\operatorname{flag}\left(E_{2}\left(P_{k}\right)\right): k=1,2, \cdots, K_{1}\right\}=\operatorname{flag}\left(E_{2}\left(P_{K_{1}}\right)\right)=N_{1}+1<\left\|\mathscr{A}_{L}\left(f_{\mathcal{G}_{1}}\right)\right\|_{L^{\infty}(Q)}^{2}+1 .
$$

Next we consider the chain $\mathscr{L}_{j}, j=2,3, \cdots, M_{0}$. When $\left\|\mathscr{A}_{L}\left(f_{\mathcal{G}_{1}}\right)\right\|_{L^{\infty}\left(R_{j}\right)} \neq 0$, we use the algorithm as in $\mathscr{L}_{1}$ above to classify cubes in the chain $\mathscr{L}_{j}$. For each $E_{2}(P)$ in the chain $\mathscr{L}_{j}$, flag $\left(E_{2}(P)\right)$ is obtained. Observe that if $E_{2}\left(P_{k}\right)$ is a common one contained in two chains, then all $E_{2}\left(P_{\ell}\right)$, 
$\ell=1, \ldots, k$, are common one. This fact, together with our algorithm above, determines that one can verify that the function flag: $\mathcal{G}_{1} \rightarrow \mathbb{N}_{+}$is well-defined and single-valued.

From the above algorithm, we know that for every $E_{2}(P) \in \mathcal{G}_{1}$, it can be assigned a label $\operatorname{flag}\left(E_{2}(P)\right)$, and

$$
N_{0}:=\max \left\{\operatorname{flag}\left(E_{2}(P)\right): E_{2}(P) \in \mathcal{G}_{1}\right\}<\left\|\mathscr{A}_{L}\left(f_{\mathcal{G}_{1}}\right)\right\|_{L^{\infty}(Q)}^{2}+1 .
$$

For $1 \leq i \leq N_{0}$, denote

$$
\mathcal{G}_{(i)}=\left\{E_{2}(P) \in \mathcal{G}_{1}: \quad \operatorname{flag}\left(E_{2}(P)\right)=i\right\}
$$

Define

$$
f_{\mathcal{G}_{(i)}}=\sum_{E_{2}(P) \in \mathcal{G}_{(i)}} a_{P}(f), \quad 1 \leq i \leq N_{0}
$$

which yields

$$
f_{\mathcal{G}_{1}}=\sum_{i=1}^{N_{0}} f_{\mathcal{G}_{(i)}}
$$

Set

$\mathcal{B}_{(j)}=\left\{E_{2}(P) \in \mathcal{G}_{(j)}: \forall E_{2}(R) \in \mathcal{G}_{(j)}\right.$, either $E_{2}(R) \in C h^{(\ell)}\left(E_{2}(P)\right)$ for $\ell \geq 0$ or $\left.E_{2}(R) \cap E_{2}(P)=\emptyset\right\}$.

That is, $\mathcal{B}_{(j)}$ is the set of maximal cubes in $\mathcal{G}_{(j)}$. With the notation above, we claim that:

(1) For every $1 \leq j \leq N_{0}$,

$$
\left\|\mathscr{A}_{L}\left(f_{\mathcal{G}_{(j)}}\right)\right\|_{L^{\infty}(Q)} \leq 2
$$

(2) There exists constant $C_{8}=C_{8}(n, \rho)>0$ such that for every $R \in \mathcal{B}_{(k)}, 2 \leq k \leq N_{0}$,

$$
\left|\left(\sum_{j=1}^{k-1} f_{\mathcal{G}_{(j)}}(x)\right)-\left(\sum_{j=1}^{k-1} f_{\mathcal{G}_{(j)}}\right)_{R}\right| \leq C_{8} \text {, a.e. } x \in R \in \mathcal{B}_{(k)} \text {. }
$$

The proof of (4.21) is simple. Indeed, since for every $x \in Q$, there exists a unique $R_{i} \in C h^{\left(K_{\mathcal{G}}-k_{0}\right)}(Q)$, $1 \leq i \leq M_{0}$ such that $x \in R_{i}$, and $\mathscr{A}_{L}\left(f_{\mathcal{G}_{(j)}}\right)(x)=\left\|\mathscr{A}_{L}\left(f_{\mathcal{G}_{(j)}}\right)\right\|_{L^{\infty}\left(R_{i}\right)}$. It follows from the above algorithm that

$$
\mathscr{A}_{L}\left(f_{\mathcal{G}_{(j)}}\right)(x)=\left(\sum_{\text {flag }\left(E_{2}(P)\right)=j, R_{i} \subset E_{2}(P)} \frac{\left|\lambda_{P}\right|^{2}}{\mu(P)}\right)^{1 / 2} \leq 2
$$

as desired. We now verify (4.22). From (iii) of Lemma 3.1 and $K_{\mathcal{G}}<\infty$, there exists a point $\tilde{x} \in R$ such that $\sum_{j=1}^{k-1} f_{\mathcal{G}_{(j)}}(\tilde{x})=\left(\sum_{j=1}^{k-1} f_{\mathcal{G}_{(j)}}\right)_{R}$. Notice that for every $E_{2}(P) \in \mathcal{G}_{(j)}$ with $R \subset E_{2}(P)$, there exists some $\ell$ with $k-j \leq \ell \leq \min \left\{2 K_{\mathcal{G}}, K_{\mathcal{G}}-k_{0}\right\}$ such that $R \in C h^{(\ell)}\left(E_{2}(P)\right)$. Denote $R \in \mathcal{D}_{\ell_{0}}^{b}$ and so $E_{2}(P) \in \mathcal{D}_{\ell_{0}-\ell}^{b}$. Note that $E_{2}(P) \in \mathcal{G}_{1}$ means that $\left|\lambda_{P}\right|^{2} / \mu(P)<1$. Therefore, one can apply (iii) of Lemma 3.1 to show that for every $j=1,2, \cdots, k-1$ and $x \in R$,

$$
\begin{aligned}
\left|f_{\mathcal{G}_{(j)}}(x)-f_{\mathcal{G}_{(j)}}(\tilde{x})\right| & \leq \sum_{\substack{E_{2}(P) \in \mathcal{G}_{(j)} \\
R \subset E_{2}(P)}}\left|a_{P}(f)(x)-a_{P}(f)(\tilde{x})\right| \\
& \leq C \sum_{\substack{k-j \leq \ell \leq 2 K_{\mathcal{G}} \\
E_{2}(P) \in \mathcal{G}_{(j)}, R \in C h^{(\ell)}\left(E_{2}(P)\right)}}\left(\frac{d\left(x, x^{\prime}\right)}{\delta^{\ell_{0}-\ell+1}}\right)^{\theta} \frac{\lambda_{P}}{\mu(P)^{1 / 2}} \\
& \leq C \sum_{\ell \geq k-j} \delta^{\theta(\ell-1)}
\end{aligned}
$$




$$
\leq \frac{C}{\sqrt{1-\delta^{2 \theta}}} \delta^{\theta(k-j-1)},
$$

where the third inequality holds by using the fact that for $R \in C h^{(\ell)}\left(E_{2}(P)\right)$, it follows from Proposition 2.2 that for $x, x^{\prime} \in R$,

$$
\frac{d\left(x, x^{\prime}\right)}{\delta^{\ell_{0}-\ell+1}} \leq C \delta^{-1} \frac{\delta^{\ell_{0}}}{\delta^{\ell_{0}-\ell}} \leq C \delta^{\ell-1} .
$$

Hence,

$$
\operatorname{LHS} \text { of (4.22) } \leq \sum_{j=1}^{k-1}\left|f_{\mathcal{G}_{(j)}}(x)-f_{\mathcal{G}_{(j)}}(\tilde{x})\right| \leq \frac{C}{\sqrt{1-\delta^{2 \theta}}} \sum_{j=1}^{k-1} \delta^{\theta(k-j-1)} \leq \frac{C}{\left(1-\delta^{\theta}\right) \sqrt{1-\delta^{2 \theta}}} .
$$

This finishes the proof of (4.22) with $C_{8}=\frac{C}{\left(1-\delta^{\theta}\right) \sqrt{1-\delta^{2 \theta}}}$.

Next we apply (4.21) and (4.22) to derive the estimate (4.16) by the iteration method. When $R_{(k)} \in \mathcal{B}_{(k)}, k=1,2, \cdots, N_{0}$, we apply (4.6) and (4.21) to obtain

$$
\int_{R_{(k)}} \exp \left(c_{4}\left|f_{\mathcal{G}_{(k)}}\right|\right) d \mu(x) \leq C_{2} \mu\left(R_{(k)}\right) \text {. }
$$

This, in combination with (4.22), implies that for any $k \in\left\{2,3, \cdots, N_{0}\right\}$,

$$
\begin{aligned}
& \int_{R_{(k)}} \exp \left(c_{4}\left|\sum_{j=1}^{k} f_{\mathcal{G}_{(j)}}(x)\right|\right) d \mu(x) \\
& \leq \exp \left(c_{4}\left|\sum_{j=1}^{k-1} f_{\mathcal{G}_{(j)}}\right|_{R_{(k)}}\right) \int_{R_{(k)}} \exp \left(c_{4}\left|\left(\sum_{j=1}^{k-1} f_{\mathcal{G}_{(j)}}(x)\right)-\left(\sum_{j=1}^{k-1} f_{\mathcal{G}_{(j)}}\right)_{R_{(k)}}\right|+c_{4}\left|f_{\mathcal{G}_{(k)}}(x)\right|\right) d \mu(x) \\
& \text { (4.24) } \leq C_{2} \exp \left(c_{4} C_{8}\right) \exp \left(c_{4}\left|\sum_{j=1}^{k-1} f_{\mathcal{G}_{(j)}}\right|_{R_{(k)}}\right) \mu\left(R_{(k)}\right) .
\end{aligned}
$$

For $x \in Q \backslash \bigcup_{R_{(k)} \in \mathcal{B}_{(k)}} R_{(k)}$, we have that $f_{\mathcal{G}_{(k)}}(x)=0$ since supp $a_{P}(f) \subset E_{2}(P)$. Then (4.24), together with the convexity of the exponential function, implies

$$
\begin{aligned}
\int_{Q} \exp \left(c_{4}\left|\sum_{j=1}^{k} f_{\mathcal{G}_{(j)}}(x)\right|\right) d \mu(x)= & \sum_{R_{(k)} \in \mathcal{B}_{(k)}} \int_{R_{(k)}} \exp \left(c_{4}\left|\sum_{j=1}^{k} f_{\mathcal{G}_{(j)}}(x)\right|\right) d \mu(x) \\
& +\int_{Q \backslash \cup_{R_{(k)} \in \mathcal{B}_{(k)}} R_{(k)}} \exp \left(c_{4}\left|\sum_{j=1}^{k-1} f_{\mathcal{G}_{(j)}}(x)\right|\right) d \mu(x) \\
\leq & C_{2} \exp \left(c_{4} C_{8}\right) \int_{Q} \exp \left(c_{4}\left|\sum_{j=1}^{k-1} f_{\mathcal{G}_{(j)}}(x)\right|\right) d \mu(x) .
\end{aligned}
$$

Recall that $C_{4}$ is the constant given in (4.9), $C_{2}=\exp \left(8 C_{4} c_{1}\right)$, and $\left\|\mathscr{A}_{L}\left(f_{\mathcal{G}_{1}}\right)\right\|_{L^{\infty}(\mathcal{Q})}^{2}>N_{0}-1$. By iteration, we have

$$
\begin{aligned}
\int_{Q} \exp \left(c_{4}\left|f_{\mathcal{G}_{1}}(x)\right|\right) d \mu(x) & =\int_{Q} \exp \left(c_{4}\left|\sum_{j=1}^{N_{0}} f_{\mathcal{G}_{(j)}}(x)\right|\right) d \mu(x) \\
& \leq C_{2}^{N_{0}-1} \exp \left(c_{4}\left(N_{0}-1\right) C_{8}\right) \int_{Q} \exp \left(c_{4}\left|f_{\mathcal{G}_{(1)}}(x)\right|\right) d \mu(x)
\end{aligned}
$$




$$
\begin{aligned}
& \leq C_{2}^{N_{0}} \exp \left(c_{4}\left(N_{0}-1\right) C_{8}\right) \mu(Q) \\
& \leq C_{2} \exp \left\{c_{4}\left(32 C_{4}+C_{8}\right)\left(N_{0}-1\right)\right\} \mu(Q) \\
& \leq C_{2} \exp \left(c_{4}\left(32 C_{4}+C_{8}\right)\left\|\mathscr{A}_{L}\left(f_{\mathcal{G}_{1}}\right)\right\|_{L^{\infty}(Q)}^{2}\right) \mu(Q) .
\end{aligned}
$$

Finally, we set $C_{7}=32 C_{4}+C_{8}+C_{1}$ to apply (4.18) and (4.26) to obtain

$$
\begin{aligned}
\int_{Q} \exp \left(c_{4}\left|f_{\mathcal{G}}(x)\right|\right) d \mu(x) & \leq \int_{Q} \exp \left(c_{4}\left(\left|f_{\mathcal{G}_{1}}(x)\right|+C_{1}\left\|\mathscr{A}_{L}\left(f_{\mathcal{G}}\right)\right\|_{L^{\infty}(Q)}^{2}\right)\right) d \mu(x) \\
& \leq C_{2} \exp \left(c_{4} C_{7}\left\|\mathscr{A}_{L}\left(f_{\mathcal{G}}\right)\right\|_{L^{\infty}(Q)}^{2}\right) \mu(Q) .
\end{aligned}
$$

From Cases (1) and (2), we have obtained (4.16). The proof of Theorem 4.2 is complete.

As a consequence, we have the following corollary.

Corollary 4.3. For a fixed $Q \in \mathcal{D}^{b}$ for some $b \in\{1,2, \cdots, K\}$, and $f_{\mathcal{G}}$ be given in (4.1). Let $0<p<\infty$. There exists a positive constant $C=C(p, n, \rho)$ independent of $\mathcal{G}, K_{\mathcal{G}}, Q$ and $f$ such that for every nonnegative $V \in L_{\text {loc }}^{1}(X)$ with $\int_{Q} V(x) d \mu(x) \neq 0$,

$$
\int_{Q}\left|f_{\mathcal{G}}(x)\right|^{p} V(x) d \mu(x) \leq C\left\|\mathscr{A}_{L}\left(f_{\mathcal{G}}\right)\right\|_{L^{\infty}(Q)}^{p} \int_{Q} V(x)\left(\log \left(e+\frac{V(x)}{V_{Q}}\right)\right)^{p / 2} d \mu(x) .
$$

Proof. From (4.15), we have that $\left(\frac{\sqrt{c_{3}}\left|f_{\mathcal{G}}\right|}{\left\|\mathscr{A}_{L}\left(f_{\mathcal{G}}\right)\right\|_{L^{\infty}(Q)}}\right)^{p} \in \operatorname{Exp}_{C_{6}}(Q, 2 / p)$ for any $0<p<\infty$, where $c_{3}$ and $C_{6}$ are constants in (4.15) and

$$
\operatorname{Exp}_{C_{6}}(Q, 1 / \gamma)=\left\{\psi: \frac{1}{\mu(Q)} \int_{Q} \exp \left(\psi^{1 / \gamma}(x)\right) d \mu(x) \leq C_{6}+1\right\} .
$$

We follow [48, Theorem 11.2] to see that if $V_{Q} \neq 0$, then for all $\gamma>0$,

$$
\int_{Q} V(x)\left(\log \left(C_{6}+\frac{V(x)}{V_{Q}}\right)\right)^{\gamma} d \mu(x) \sim \sup \left\{\int_{Q} V(x) \phi(x) d \mu(x): \phi \in \operatorname{Exp}_{C_{6}}(Q, 1 / \gamma)\right\},
$$

with comparability constants that only depend on $\gamma$.

By (4.29) with $\gamma=2 / p$,

$$
\int_{Q}\left(\frac{\sqrt{c_{3}}\left|f_{\mathcal{G}}\right|}{\left\|\mathscr{A}_{L}\left(f_{\mathcal{G}}\right)\right\|_{L^{\infty}(Q)}}\right)^{p} V(x) d \mu(x) \leq C_{p} \int_{Q} V(x)\left(\log \left(C_{6}+\frac{V(x)}{V_{Q}}\right)\right)^{p / 2} d \mu(x) .
$$

Meanwhile, there exists constant $C=C\left(C_{6}, p\right)$ independent of $V$ and $Q$, such that

$$
\int_{Q} V(x)\left(\log \left(C_{6}+\frac{V(x)}{V_{Q}}\right)\right)^{p / 2} d \mu(x) \leq C \int_{Q} V(x)\left(\log \left(e+\frac{V(x)}{V_{Q}}\right)\right)^{p / 2} d \mu(x) .
$$

Hence (4.28) holds, and we complete the proof of Corollary 4.3.

We are now ready to prove the following theorem.

Theorem 4.4. Suppose that $L$ is a densely-defined operator on $L^{2}(X)$ satisfying $(\boldsymbol{H 1}),(\boldsymbol{H} 2)$ and $(\boldsymbol{H 3})$. Let $f \in L^{2}(X, d \mu)$ and assume $S_{L, \alpha} f \in L^{\infty}(X)$. Then there exist two positive constants $c_{5}=c_{5}(n, \alpha)$, $C_{9}=C_{9}(n, \alpha)$ independent of $f$ such that

$$
\sup _{B} \frac{1}{\mu(B)} \int_{B} \exp \left(c_{5} \frac{\left|f(x)-f_{B}\right|^{2}}{\left\|S_{L, \alpha} f\right\|_{L^{\infty}(X)}^{2}}\right) d \mu(x) \leq C_{9},
$$

where the supremum takes for all balls $B$. 
Proof. To prove Theorem 4.4, one can apply Lemma 3.2 and fix the parameter $\rho=\delta^{2} \alpha / 5$ to obtain that it suffices to show

$$
\sup _{B} \frac{1}{\mu(B)} \int_{B} \exp \left(c^{2} c_{5} \frac{\left|f(x)-f_{B}\right|^{2}}{\left\|\mathscr{A}_{L}(f)\right\|_{L^{\infty}(X)}^{2}}\right) d \mu(x) \leq C_{9},
$$

where $c$ is the constant in (3.6).

Let us show (4.31). Since $f \in L^{2}(X)$, the Calderón reproducing formula (3.1) tells us

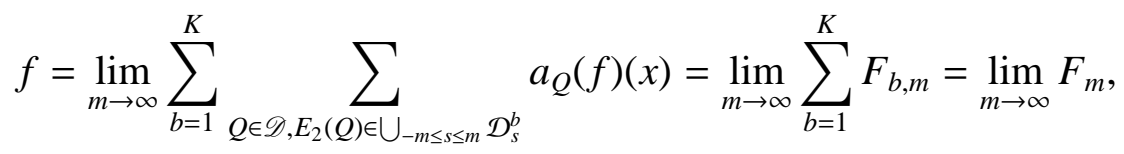

where the limits converge in $L^{2}(X)$. Then one can apply the Riesz theorem to see there exists some subsequence $\left\{m_{i}\right\}$ such that $\lim _{i \rightarrow \infty} F_{m_{i}}(x)=f(x)$, a.e. $x \in X$. Also for every ball $B$,

$$
\lim _{m \rightarrow \infty}\left|f_{B}-\left(F_{m}\right)_{B}\right| \leq \lim _{m \rightarrow \infty}\left(\frac{1}{\mu(B)} \int_{B}\left|f(x)-F_{m}(x)\right|^{2} d \mu(x)\right)^{1 / 2}=0 .
$$

It follows from the fact that $\mathscr{A}_{L}\left(F_{b, m}\right)(x) \leq \mathscr{A}_{L}\left(F_{m}\right)(x) \leq \mathscr{A}_{L}(f)(x)$, a.e. $x \in X$,

$$
\frac{\left|f(x)-f_{B}\right|^{2}}{\left\|\mathscr{A}_{L}(f)\right\|_{L^{\infty}(X)}^{2}} \leq 3 \frac{\left|f(x)-F_{m_{i}}(x)\right|^{2}}{\left\|\mathscr{A}_{L}(f)\right\|_{L^{\infty}(X)}^{2}}+3 \frac{\left|F_{m_{i}}(x)-\left(F_{m_{i}}\right)_{B}\right|^{2}}{\left\|\mathscr{A}_{L}\left(F_{m_{i}}\right)\right\|_{L^{\infty}(X)}^{2}}+3 \frac{\left|\left(F_{m_{i}}\right)_{B}-f_{B}\right|^{2}}{\left\|\mathscr{A}_{L}(f)\right\|_{L^{\infty}(X)}^{2}},
$$

which implies

$$
\frac{\left|f(x)-f_{B}\right|^{2}}{\left\|\mathscr{A}_{L}(f)\right\|_{L^{\infty}(X)}^{2}} \leq 3 \lim _{i \rightarrow \infty} \frac{\left|F_{m_{i}}(x)-\left(F_{m_{i}}\right)_{B}\right|^{2}}{\left\|\mathscr{A}_{L}\left(F_{m_{i}}\right)\right\|_{L^{\infty}(X)}^{2}}
$$

From Fatou's lemma,

$$
\begin{aligned}
\int_{B} \exp \left(c^{2} c_{5} \frac{\left|f(x)-f_{B}\right|^{2}}{\left\|\mathscr{A}_{L}(f)\right\|_{L^{\infty}(X)}^{2}}\right) d \mu(x) & \leq C \frac{\lim }{i \rightarrow \infty} \int_{B} \exp \left(3 c^{2} c_{5} \frac{\left|F_{m_{i}}(x)-\left(F_{m_{i}}\right)_{B}\right|^{2}}{\left\|\mathscr{A}_{L}\left(F_{m_{i}}\right)\right\|_{L^{\infty}(X)}^{2}}\right) d \mu(x) \\
& \leq \frac{\lim }{i \rightarrow \infty} \frac{C}{K} \sum_{b=1}^{K} \int_{B} \exp \left(3 K^{2} c^{2} c_{5} \frac{\left|F_{b, m_{i}}(x)-\left(F_{b, m_{i}}\right)_{B}\right|^{2}}{\left\|\mathscr{A}_{L}\left(F_{b, m_{i}}\right)\right\|_{L^{\infty}(X)}^{2}}\right) d \mu(x) .
\end{aligned}
$$

Therefore, it suffices to show for every ball $B$ and $F_{b, m}, b \in\{1,2, \cdots, K\}, m>0$, there exist two positive constants $c_{6}$ and $C_{10}$ independent of $F_{b, m}$ and $B$, such that

$$
\frac{1}{\mu(B)} \int_{B} \exp \left(c_{6} \frac{\left|F_{b, m}(x)-\left(F_{b, m}\right)_{B}\right|^{2}}{\left\|\mathscr{A}_{L}\left(F_{b, m}\right)\right\|_{L^{\infty}(X)}^{2}}\right) d \mu(x) \leq C_{10}
$$

Let us prove (4.33). Assume $\operatorname{diam} B \sim \delta^{k_{0}}$. Define $\mathcal{G}_{b, m}=\left\{Q \in \mathcal{D}_{k_{0}}^{b}: Q \cap B \neq \emptyset\right\}$. Obviously, $\# \mathcal{G}_{b, m} \leq M_{1}$ for some $M_{1}$ depending on $n$ and $\delta$ only. Then for $x \in B$, by $\operatorname{supp} a_{P} \subset E_{2}(P)$, we write

$$
\begin{aligned}
& F_{b, m}(x)=\sum_{Q \in \mathcal{G}_{b, m}} \sum_{\substack{E_{2}(P) \subset Q \\
E_{2}(P) \in \bigcup_{-m \leq s \leq m} \mathcal{D}_{s}^{b}}} a_{P}(f)(x)+\sum_{Q \in \mathcal{G}_{b, m}} \sum_{\substack{Q \subsetneq E_{2}(P) \\
E_{2}(P) \in \bigcup_{-m \leq s \leq m} \mathcal{D}_{s}^{b}}} a_{P}(f)(x) \\
& =: \quad \sum_{Q \in \mathcal{G}_{b, m}} F_{Q, 1}(x)+\sum_{Q \in \mathcal{G}_{b, m}} F_{Q, 2}(x) \text {. }
\end{aligned}
$$


Note that $\mathscr{A}_{L}\left(F_{Q, 1}\right)(x) \leq \mathscr{A}_{L}\left(F_{b, m}\right)(x)$ and $\mathscr{A}_{L}\left(F_{Q, 2}\right)(x) \leq \mathscr{A}_{L}\left(F_{b, m}\right)(x)$. By the convexity of the exponential function,

$$
\text { LHS of (4.33) } \leq \frac{1}{2 M_{1}} \sum_{Q \in \mathcal{G}_{b, m}} \sum_{j=1}^{2} \frac{1}{\mu(B)} \int_{B} \exp \left(4 M_{1}^{2} c_{6} \frac{\left|F_{Q, j}(x)-\left(F_{Q, j}\right)_{B}\right|^{2}}{\left\|\mathscr{A}_{L}\left(F_{Q, j}\right)\right\|_{L^{\infty}(X)}^{2}}\right) d \mu(x) .
$$

It reduces to show for every ball $B$ and $F_{Q, j}, j=1,2$, there exist two positive constants $c_{7}$ and $C_{11}$ independent of $F_{Q, j}$ and $B$, such that

$$
\frac{1}{\mu(B)} \int_{B} \exp \left(c_{7} \frac{\left|F_{Q, j}(x)-\left(F_{Q, j}\right)_{B}\right|^{2}}{\left\|\mathscr{A}_{L}\left(F_{Q, j}\right)\right\|_{L^{\infty}(X)}^{2}}\right) \leq C_{11} .
$$

Consider the case $j=1$. We note that $\operatorname{supp} F_{Q, 1} \subset Q$. A similar approach to derive (4.3) gives

$$
\left|F_{Q, 1}\right|_{B}^{2} \leq \frac{C}{\mu(Q)} \int_{Q}\left|F_{Q, 1}(x)\right|^{2} d \mu(x) \leq C C_{1}^{2}\left\|\mathscr{A}_{L}\left(F_{Q, 1}\right)\right\|_{L^{\infty}(Q)}^{2},
$$

where $C_{1}$ is the constant in (4.3). Then again note that $\operatorname{supp} F_{Q, 1} \subset Q$,

$$
\begin{aligned}
& \frac{1}{\mu(B)} \int_{B} \exp \left(c_{7} \frac{\left|F_{Q, 1}(x)-\left(F_{Q, 1}\right)_{B}\right|^{2}}{\left\|\mathscr{A}_{L}\left(F_{Q, 1}\right)\right\|_{L^{\infty}(X)}^{2}}\right) d \mu(x) \\
& \leq \frac{C \exp \left(C C_{1}^{2}\right)}{\mu(Q)} \sum_{Q^{\prime} \in \mathcal{G}_{b, m}} \int_{Q^{\prime}} \exp \left(2 c_{7} \frac{\left|F_{Q, 1}(x)\right|^{2}}{\left\|\mathscr{A}_{L}\left(F_{Q, 1}\right)\right\|_{L^{\infty}(Q)}^{2}}\right) d \mu(x) \\
& \leq \frac{C \exp \left(C C_{1}^{2}\right)}{\mu(Q)} \int_{Q} \exp \left(2 c_{7} \frac{\left|F_{Q, 1}(x)\right|^{2}}{\left\|\mathscr{A}_{L}\left(F_{Q, 1}\right)\right\|_{L^{\infty}(Q)}^{2}}\right) d \mu(x)+\left(M_{1}-1\right) C \exp \left(C C_{1}^{2}\right) .
\end{aligned}
$$

This, together with Theorem 4.2, deduces that there exists a constant $C_{10}$ independent on $F_{Q, 1}$ and $B$, such that

$$
\frac{1}{\mu(B)} \int_{B} \exp \left(c_{7} \frac{\left|F_{Q, 1}(x)-\left(F_{Q, 1}\right)_{B}\right|^{2}}{\left\|\mathscr{A}_{L}\left(F_{Q, 1}\right)\right\|_{L^{\infty}(X)}^{2}}\right) d \mu(x) \leq C_{6} M_{1} C \exp \left(C C_{1}^{2}\right) \leq C_{11}
$$

for $c_{7} \leq c_{3} / 2$, where $c_{3}$ is the constant in (4.15). This proves (4.34) for the case $j=1$.

Next we work the case $j=2$. Note that $F_{Q, 2}$ is continuous on $B$, so there exists $x^{\prime} \in B$ such that $\left(F_{Q, 2}\right)_{B}=F_{Q, 2}\left(x^{\prime}\right)$. A similar argument as in the proof of (4.7) shows that

$$
\left|F_{Q, 2}(x)-\left(F_{Q, 2}\right)_{B}\right|=\left|F_{Q, 2}(x)-F_{Q, 2}\left(x^{\prime}\right)\right| \leq C_{3}\left\|\mathscr{A}_{L}\left(F_{Q, 2}\right)\right\|_{L^{\infty}(Q)}, \quad x \in B,
$$

where $C_{3}$ is the constant in (4.7). Then

$$
\frac{1}{\mu(B)} \int_{B} \exp \left(c_{7} \frac{\left|F_{Q, 2}(x)-\left(F_{Q, 2}\right)_{B}\right|^{2}}{\left\|\mathscr{A}_{L}\left(F_{Q, 2}\right)\right\|_{L^{\infty}(X)}^{2}}\right) d \mu(x) \leq \exp \left(\gamma_{3} C_{3}^{2}\right) \leq C_{11},
$$

which proves (4.34) for the case $j=2$ and finishes the proof of Theorem 4.4.

Remarks. We would like to comment on the possibility of several generalizations and open problems related to the results of Theorem 4.4.

(1) The first one is the extension of the work of Chang-Wilson-Wolff ([8, Theorem 3.2]) to nonhomogeneous metric spaces, in place of $\mathbb{R}^{n}$ (see $[44,45]$ for the definition and properties of nonhomogeneous metric spaces), and this question will be considered in the future.

(2) In the proof of Theorem 4.4, a key step is to use Theorem 4.2, which provides an algorithm to obtain a direct proof of its exponential-square integrability of a function whose discrete square function associated to an operator $L$ is bounded. In the proof, we used the assumption (H3) of 
Hölder's continuity in $x$ of the kernels $p_{t}(x, y)$ of $e^{-t L}$. We may ask whether Theorem 4.4 still holds assuming merely that an operator $L$ satisfies (H1) and (H2). This problem continues a line of the study of harmonic analysis properties of abstract selfadjoint operators whose kernel satisfies weaker conditions than standard regularity estimates, and there was lots of success in solving this problem in the last few years see for example, in $[1,2,3,5,16,17,18,19,27]$ and the references therein.

\section{Singular INTEGRAL OPERATORS}

In this section, we apply the previous results in Section 4 to obtain some estimates of the norms on $L^{p}$ as $p$ becomes large for operators such as the square functions or spectral multipliers, and weighted norm inequalities for the square functions.

5.1. Square function results. In this subsection we establish sharp lower bounds for the operator norm of the square functions on $L^{p}(X, d \mu)$ as $p$ becomes large.

Proposition 5.1. Let $(X, d, \mu)$ be a metric measure space endowed with a distance $d$ and a nonnegative Borel doubling measure $\mu$ on $X$. Assume that $L$ is a densely-defined operator on $L^{2}(X)$ satisfying (H1), (H2) and (H3). Then there exists a positive constant $C=C(n, \rho)$ such that

$$
\|f\|_{L^{p}(X, d \mu)} \leq C p^{1 / 2}\left\|\mathscr{A}_{L}(f)\right\|_{L^{p}(X, d \mu)} \text { as } p \rightarrow \infty .
$$

Proof. The proof of (5.1) is based on combing a technique of [48, Theorem 11.3] and the FeffermanPipher's method [23]. Notice that

$$
\|f\|_{L^{p}(X, d \mu)}=\sup _{\|V\|_{L^{p^{\prime}(X, d \mu)}}} \int_{X} f(x) V(x) d \mu(x), \quad 1 / p+1 / p^{\prime}=1,
$$

it suffices to show that there exists a constant $C>0$ such that

$$
\left|\int_{X} f(x) V(x) d \mu(x)\right| \leq C p^{1 / 2}\left\|\mathscr{A}_{L}(f)\right\|_{L^{p}(X, d \mu)}, \quad\|V\|_{L^{p^{\prime}(X, d \mu)}}=1
$$

as $p \rightarrow \infty$.

Next we decompose $f$ and $V$ to $f_{k}$ and $V_{k}$ such that $f_{k}, V_{k} \in L^{2}(X)$ and so Calderón reproducing formula can be applied. Fix a sequence of sets $E_{1} \subset E_{2} \subset \cdots \subset E_{k} \subset E_{k+1} \subset \cdots \subset X$ satisfying $\mu\left(E_{k}\right)<\infty$ for every $k \geq 1$ and $X=\bigcup_{k} E_{k}$. For every $f \in L^{p}(X, d \mu), 2 \leq p<\infty$, let $f_{k}=f \chi_{E_{k}}$, $k=1,2, \cdots$. It's clear that $f_{k} \in L^{2}(X, d \mu) \cap L^{p}(X, d \mu)$ and $\lim _{k \rightarrow \infty}\left\|f-f_{k}\right\|_{L^{p}(X, d \mu)}=0$. Notice that $L_{0}^{\infty}(X)$ is densely contained in $L^{q}(X)$ for every $1<q<\infty$, where $L_{0}^{\infty}(X)$ denotes the space of bounded functions with bounded support. Therefore for every $V \in L^{p^{\prime}}(X, d \mu)$ with $\|V\|_{L^{p^{\prime}(X, d \mu)}}=1$, there exists $\left\{V_{k}\right\}_{k=1}^{\infty} \subset L_{0}^{\infty}(X, d \mu)$ such that $\lim _{k \rightarrow \infty}\left\|V-V_{k}\right\|_{L^{p^{\prime}(X, d \mu)}}=0$. In the sequel, we may assume $\left\|V_{k}\right\|_{L^{p^{\prime}}(X, d \mu)} \leq 2$ for all $k \geq 1$.

For $f \in L^{p}(X)$ and $V \in L^{p^{\prime}}(X)$, note that $f_{k}, V_{k} \in L^{2}(X, d \mu)$, one can apply Calderón reproducing formula (3.1) to deduce

$$
\begin{aligned}
\int_{X} f(x) V(x) d \mu(x) & =\lim _{k \rightarrow \infty} \int_{X} f_{k}(x) V_{k}(x) d \mu(x) \\
& =\lim _{k \rightarrow \infty} \lim _{m \rightarrow \infty} \sum_{b=1}^{K} \int_{X} F_{b, m}(x) V_{k}(x) d \mu(x),
\end{aligned}
$$


where

$$
F_{b, m}(x):=\sum_{\substack{Q \in \mathscr{D} \\ E_{2}(Q) \in \cup_{-m \leq s \leq m} \mathcal{D}_{s}^{b}}} a_{Q}\left(f_{k}\right)(x) .
$$

For $F_{b, m}$ and $V_{k}$ defined above, it turns to estimate $\int\left|F_{b, m}(x) \| V_{k}(x)\right| d \mu(x)$. To this end, we apply the technique in [48, Theorem 11.3]. For every given $b \in\{1,2, \cdots, K\}$ and $m \geq 1$, set $E_{b, m}^{j}=$ $\left\{x \in X: \mathscr{A}_{L}\left(F_{b, m}\right)(x)>2^{j}\right\}$ and

$$
D_{b, m}^{j}=\left\{E_{2}(Q): Q \in \mathscr{D}, E_{2}(Q) \in \bigcup_{-m \leq s \leq m} \mathcal{D}_{s}^{b}, E_{2}(Q) \subset E_{b, m}^{j}, E_{2}(Q) \not \subset E_{b, m}^{j+1}\right\}, j \in \mathbb{Z} .
$$

Note that function $\mathscr{A}_{L}\left(F_{b, m}\right)(x)$ just takes finite values on each dyadic cube and so can take minimal value on each dyadic cube. It is easy to see that

$$
D_{b, m}^{j}=\left\{E_{2}(Q): Q \in \mathscr{D}, E_{2}(Q) \in \bigcup_{-m \leq s \leq m} \mathcal{D}_{s}^{b}, 2^{j}<\min _{x \in E_{2}(Q)} \mathscr{A}_{L}\left(F_{b, m}\right)(x) \leq 2^{j+1}\right\}, j \in \mathbb{Z} .
$$

Sets $D_{b, m}^{j}$ for different $j$ are disjoint (some of them might be empty) and

$$
\left\{E_{2}(Q): Q \in \mathscr{D}, E_{2}(Q) \in \bigcup_{-m \leq s \leq m} \mathcal{D}_{s}^{b}\right\}=\bigcup_{j} D_{b, m}^{j} .
$$

Set

$$
F_{b, m}^{j}(x)=\sum_{E_{2}(Q) \in D_{b, m}^{j}} a_{Q}\left(f_{k}\right)(x), \quad j \in \mathbb{Z}
$$

Then $F_{b, m}=\sum_{j} F_{b, m}^{j}$.

We claim that $\mathscr{A}_{L}\left(F_{b, m}^{j}\right) \leq 2^{j+1}$ everywhere. Indeed, if $\mathscr{A}_{L}\left(F_{b, m}^{j}\right)\left(x_{0}\right)>2^{j+1}$ for some $x_{0} \in X$, then there exists $E_{2}(Q) \in D_{b, m}^{j}$ such that $x_{0} \in E_{2}(Q)$; otherwise $F_{b, m}^{j}\left(x_{0}\right)=0$. Let $E_{2}\left(Q_{0}\right)$ be the minimal cube in $\left\{E_{2}(Q) \in D_{b, m}^{j}, x_{0} \in E_{2}(Q)\right\}$, it's clear that $\mathscr{A}_{L}\left(F_{b, m}^{j}\right)(x)=\mathscr{A}_{L}\left(F_{b, m}^{j}\right)\left(x_{0}\right)>2^{j+1}$ for all $x \in E_{2}\left(Q_{0}\right)$. Therefore, $E_{2}\left(Q_{0}\right) \subset E_{b, m}^{j+1}$ follows from $\mathscr{A}_{L}\left(F_{b, m}\right) \geq \mathscr{A}_{L}\left(F_{b, m}^{j}\right)$, which is in conflict with $E_{2}\left(Q_{0}\right) \in D_{b, m}^{j}$.

Set

$$
\mathcal{B}_{b, m}^{j}=\left\{Q_{b, m}^{j}: Q_{b, m}^{j} \text { is the maximal dyadic cube in } D_{b, m}^{j}\right\},
$$

It's clear that any two cubes in $\mathcal{B}_{b, m}^{j}$ are disjoint. Besides it is easy to check

$$
\operatorname{supp} F_{b, m}^{j} \subset \bigcup_{Q_{b, m}^{j} \in \mathcal{B}_{b, m}^{j}} Q_{b, m}^{j} \subset E_{b, m}^{j} .
$$

Let $M$ be the classical Hardy-Littlewood maximal operator. Following R. Fefferman-Pipher's method [23], set

$$
\widetilde{V}_{k}=\left|V_{k}\right|+\frac{M\left(V_{k}\right)}{2\|M\|_{L^{p^{\prime}}(X, d \mu)}}+\frac{M \circ M\left(V_{k}\right)}{\left(2\|M\|_{L^{p^{\prime}}(X, d \mu)}\right)^{2}}+\cdots,
$$

where $\|M\|_{L^{p^{\prime}(X, d \mu)}}$ denotes the operator norm of the Hardy-Littlewood maximal operator on $L^{p^{\prime}}(X, d \mu)$. Then $\left\|\widetilde{V}_{k}\right\|_{L^{p^{\prime}(X, d \mu)}} \leq 4$ and $\left\|\widetilde{V}_{k}\right\|_{A_{1}} \leq 2\|M\|_{L^{p^{\prime}(X, d \mu)}}$, where $A_{1}$ is the classical Muckenhoupt weight class. 
Without loss of generality, we assume that $\left(\widetilde{V}_{k}\right)_{Q_{b, m}^{j}} \neq 0$ for all $Q_{b, m}^{j} \in \mathcal{B}_{b, m}^{j}$. Note that $\left\|\mathscr{A}_{L}\left(F_{b, m}^{j}\right)\right\|_{L^{\infty}} \leq$ $2^{j+1}$. Then one can use Corollary 4.3 with $p=1$ to see

$$
\begin{aligned}
\int_{X}\left|F_{b, m}(x)\right|\left|V_{k}(x)\right| d \mu(x) & \leq \sum_{j \in \mathbb{Z}} \sum_{Q_{b, m}^{j} \in \mathcal{B}_{b, m}^{j}} \int_{Q_{b, m}^{j}}\left|F_{b, m}^{j}(x)\right| \widetilde{V}_{k}(x) d \mu(x) \\
& \leq C \sum_{j \in \mathbb{Z}} 2^{j} \sum_{Q_{b, m}^{j} \in \mathcal{B}_{b, m}^{j}} \int_{Q_{b, m}^{j}} \widetilde{V}_{k}(x)\left(\log \left(e+\frac{\widetilde{V}_{k}(x)}{\left(\widetilde{V}_{k}\right)_{Q_{b, m}^{j}}}\right)\right)^{1 / 2} d \mu(x),
\end{aligned}
$$

By a similar approach to prove (2.15) in [48, pp. 17], we can prove there exists constant $C>0$ such that for any dyadic cube $Q$ and function $f$ defined on $Q$,

$$
\int_{Q}|f(x)| \log \left(e+\frac{|f(x)|}{|f|_{Q}}\right) d \mu(x) \leq C \int_{Q} M\left(f \chi_{Q}\right) d \mu(x) .
$$

By Hölder's inequality,

$$
\begin{aligned}
& \int_{Q_{b, m}^{j}} \widetilde{V}_{k}(x)\left(\log \left(e+\frac{\widetilde{V}_{k}(x)}{\left(\widetilde{V}_{k}\right)_{Q_{b, m}^{j}}}\right)\right)^{1 / 2} d \mu(x) \\
\leq & \left(\int_{Q_{b, m}^{j}} \widetilde{V}_{k}(x) d \mu(x)\right)^{1 / 2}\left(\int_{Q_{b, m}^{j}} \widetilde{V}_{k}(x) \log \left(e+\frac{\widetilde{V}_{k}(x)}{\left(\widetilde{V}_{k}\right)_{Q_{b, m}^{j}}}\right) d \mu(x)\right)^{1 / 2} \\
\leq & C\left(\int_{Q_{b, m}^{j}} \widetilde{V}_{k}(x) d \mu(x)\right)^{1 / 2}\left(\int_{Q_{b, m}^{j}} M\left(\widetilde{V}_{k} \chi_{Q_{b, m}^{j}}\right) d \mu(x)\right)^{1 / 2} \\
\leq & C\left\|\widetilde{V}_{k}\right\|_{A_{1}}^{1 / 2} \int_{Q_{b, m}^{j}} \widetilde{V}_{k}(x) d \mu(x) \\
\leq & C\|M\|_{L^{p^{\prime}(X, d \mu)}}^{1 / 2} \int_{Q_{b, m}^{j}} \widetilde{V}_{k}(x) d \mu(x) .
\end{aligned}
$$

Therefore,

$$
\begin{aligned}
& \int_{X}\left|F_{b, m}(x)\right|\left|V_{k}(x)\right| d \mu(x) \\
\leq & C\|M\|_{L^{p^{\prime}(X, d \mu)}}^{1 / 2} \sum_{j \in \mathbb{Z}} 2^{j} \sum_{Q_{b, m}^{j} \in \mathcal{B}_{b, m}^{j}} \int_{Q_{b, m}^{j}} \widetilde{V}_{k}(x) d \mu(x) \\
\leq & C\|M\|_{L^{p^{\prime}(X, d \mu)}}^{1 / 2} \sum_{j \in \mathbb{Z}} \sum_{Q_{b, m}^{j} \in \mathcal{B}_{b, m}^{j}} \int_{Q_{b, m}^{j}} \mathscr{A}_{L}\left(F_{b, m}\right)(x) \widetilde{V}_{k}(x) d \mu(x) \\
\leq & C\|M\|_{L^{p^{\prime}(X, d \mu)}}^{1 / 2} \int_{X} \mathscr{A}_{L}\left(F_{b, m}\right)(x) \widetilde{V}_{k}(x) d \mu(x) \\
\leq & C\|M\|_{L^{p^{\prime}(X, d \mu)}}^{1 / 2} \int_{X} \mathscr{A}_{L}\left(f_{k}\right)(x) \widetilde{V}_{k}(x) d \mu(x) \\
\leq & C\|M\|_{L^{p^{\prime}(X, d \mu)}}^{1 / 2} \int_{X}\left[\sqrt{2}_{2} \mathscr{A}_{L}\left(f-f_{k}\right)(x)+\left(1+\sqrt{2}_{2}\right) \mathscr{A}_{L}(f)(x)\right] \widetilde{V}_{k}(x) d \mu(x) \\
\leq & C\|M\|_{L^{p^{\prime}(X, d \mu)}}^{1 / 2}\left(\left\|\mathscr{A}_{L}(f)\right\|_{L^{p}(X, d \mu)}+\left\|\mathscr{A}_{L}\left(f-f_{k}\right)\right\|_{L^{p}(X, d \mu)}\right) .
\end{aligned}
$$


By (3.6),

$$
\left\|\mathscr{A}_{L}\left(f-f_{k}\right)\right\|_{L^{p}(X, d \mu)} \leq c\left\|S_{L, 5 \delta^{-2}}\left(f-f_{k}\right)\right\|_{L^{p}(X, d \mu)} \leq C_{p}\left\|f-f_{k}\right\|_{L^{p}(X, d \mu)},
$$

where $c$ be the constant in (3.6). This, together with (5.2), (5.3), (5.4)and (5.5), yields that for any $f \in L^{p}(X, d \mu), 2 \leq p<\infty$, and $\|V\|_{L^{p^{\prime}}(X, d \mu)}=1$,

$$
\begin{aligned}
\left|\int_{X} f(x) V(x) d \mu(x)\right| & \leq C\|M\|_{L^{p^{\prime}(X, d \mu)}}^{1 / 2}\left\|\mathscr{A}_{L}(f)\right\|_{L^{p}(X, d \mu)}+C_{p}\|M\|_{L^{p^{\prime}(X, d \mu)}}^{1 / 2} \lim _{k \rightarrow \infty}\left\|f-f_{k}\right\|_{L^{p}(X, d \mu)} \\
& =C\|M\|_{L^{p^{\prime}(X, d \mu)}}^{1 / 2}\left\|\mathscr{A}_{L}(f)\right\|_{L^{p}(X, d \mu)} .
\end{aligned}
$$

By [29, Theorem 1.3], we have

$$
\|M\|_{L^{q}(X, d \mu)} \leq C_{\mu}\left(\frac{1}{q-1}\right)^{1 / q}, 1<q<\infty,
$$

where $C_{\mu}$ depends only on the doubling constant of the measure $\mu$ (see also [41, pp. 7]).

Therefore,

$$
\|f\|_{L^{p}(X, d \mu)} \leq C p^{1 / 2}\left\|\mathscr{A}_{L}(f)\right\|_{L^{p}(X, d \mu)}, \text { as } p \rightarrow \infty .
$$

This finishes the proof of (5.1), and finish the proof of Proposition 5.1.

We now apply Proposition 5.1 and Lemma 3.2 to obtain the following result readily.

Theorem 5.2. Assume that $L$ is a densely-defined operator on $L^{2}(X)$ satisfying $(\boldsymbol{H 1}),(\boldsymbol{H} 2)$ and $(\boldsymbol{H} 3)$. The area function $S_{L, \alpha}$ is defined in (1.3). Then there exists a constant $C=C(n, \alpha)>0$ such that

$$
\|f\|_{L^{p}(X, d \mu)} \leq C p^{1 / 2}\left\|S_{L, \alpha}(f)\right\|_{L^{p}(X, d \mu)} \text { as } p \rightarrow \infty .
$$

5.2. $L^{p}$ bounds for spectral multipliers. Suppose that $L$ is a nonnegative self-adjoint operator acting on $L^{2}(X)$. Let $E(\lambda)$ be the spectral resolution of $L$. By the spectral theorem, for any bounded Borel function $F:[0, \infty) \rightarrow \mathbb{C}$, one can define the operator

$$
F(L)=\int_{0}^{\infty} F(\lambda) d E(\lambda)
$$

which is bounded on $L^{2}(X)$. In [17, Theorem 3.1], X.T. Duong, E.M. Ouhabaz and A. Sikora obtain the following Hörmander-type spectral multiplier result for the special case $m=2$.

Proposition 5.3. Assume that $L$ is a densely-defined operator on $L^{2}(X)$ satisfying $(\boldsymbol{H} 1)$ and $(\boldsymbol{H} 2)$. Let $\beta>n / 2$ and assume that for any $R>0$ and all Borel functions $F$ such that supp $F \subseteq[0, R]$,

$$
\int_{X}\left|K_{F(\sqrt{L})}(x, y)\right|^{2} d \mu(x) \leq \frac{C}{\mu\left(B\left(y, R^{-1}\right)\right)}\left\|\delta_{R} F\right\|_{L^{q}(\mathbb{R})}^{2}
$$

for some $q \in[2, \infty]$. Next suppose that $F:[0, \infty) \rightarrow \mathbb{C}$ is a bounded Borel function such that $F(0)=0$, $\sup _{t>0}\left\|\eta \delta_{t} F\right\|_{W_{\beta}^{q}(\mathbb{R})}<\infty$ where $\delta_{t} F(\lambda)=F(t \lambda),\|F\|_{W_{\beta}^{q}(\mathbb{R})}=\left\|\left(I-d^{2} / d x^{2}\right)^{\beta / 2} F\right\|_{L^{q}(\mathbb{R})}$ and $\eta \in C_{0}^{\infty}\left(\mathbb{R}_{+}\right)$is a fixed function, not identically zero. Then the operator $F(L)$ is bounded on $L^{p}(X)$ for all $1<p<\infty$.

It should be noted that Gaussian bounds (H2) implies estimates (5.8) for $q=\infty$. Hypothesis (5.8) is called the Plancherel estimate or the Plancherel condition. For the standard Laplace operator on Euclidean spaces $\mathbb{R}^{n}$, this is equivalent to $(1,2)$ Stein-Tomas restriction theorem (which is also the Plancherel estimate of the Fourier transform) (see $[17,11])$.

In this section we will apply the result from the previous section in order to derive certain estimates on the $L^{p}$ operator norm $p \rightarrow \infty$ of spectral multipliers $F(L)$. 
Theorem 5.4. Under the assumptions of Proposition 5.3 and the assumption in addition that $L$ satisfies (H3), the operator $F(L)$ satisfies

$$
\|F(L) f\|_{p} \leq C p \sup _{t>0}\left\|\eta \delta_{t} F\right\|_{W_{\beta}^{q}(\mathbb{R})}\|f\|_{p},
$$

as $p \rightarrow \infty$.

We will break up the proof into two parts in which we deal with the $\mathcal{G}_{\lambda}^{*}$ function: Let $\phi \in C_{0}^{\infty}(\mathbb{R})$ be even function with $\int \phi=1, \operatorname{supp} \phi \subset(-1 / 10,1 / 10)$. Let $\varphi$ denote the Fourier transform of $\phi$ and let $\psi(s)=s^{2 n+2} \varphi^{3}(s)$. We define the $\mathcal{G}_{\lambda}^{*}$ function by

$$
\mathcal{G}_{\lambda}^{*}(f)(x)=\left(\int_{0}^{\infty} \int_{X}\left(\frac{t}{t+d(x, y)}\right)^{n \lambda}|\psi(t \sqrt{L}) f(y)|^{2} \frac{d \mu(y)}{\mu(B(x, t))} \frac{d t}{t}\right)^{1 / 2}, \quad \lambda>1 .
$$

It is known that under the assumptions (H1) and (H2) of the operator $L, \mathcal{G}_{\lambda}^{*}(f)$ is bounded on $L^{p}(X)$ for all $1<p<\infty$. Following the method of Rubio de Francia and García-Cuerva (see pp. 356 - 357 , [23]), we have

$$
\left\|\mathcal{G}_{\lambda}^{*}(f)\right\|_{L^{p}(X)} \leq C p^{1 / 2}\|f\|_{L^{p}(X)} .
$$

as $p \rightarrow \infty$. See [24, Theorems 1.2 and 3.3] and [25, Theorem 1.3].

Proposition 5.5. Under the assumptions of Proposition 5.3, there exist a constant $C=C_{n, \lambda, \Psi}$ and some $\lambda>1$ such that for every $f \in L^{2}(X)$,

$$
S_{L}(F(L) f)(x) \leq C \sup _{t>0}\left\|\eta \delta_{t} F\right\|_{W_{\beta}^{q}(\mathbb{R})} \mathcal{G}_{\lambda}^{*}(f)(x) .
$$

Proof. By the spectral theory ([50]), for every $f \in L^{2}(X)$ and every $\kappa>3 n$,

$$
f=C_{\psi} \int_{0}^{\infty}\left(t^{2} L\right)^{\kappa} e^{-t \sqrt{L}} \psi(t \sqrt{L}) f \frac{d t}{t}
$$

with $C_{\psi}^{-1}=\int_{0}^{\infty} t^{2 \kappa} e^{-t} \psi(t) d t / t$, and the integral converges in $L^{2}(X)$.

$$
\begin{aligned}
s^{2} L e^{-s \sqrt{L}} F(L) f(y) & =C_{\psi} \int_{0}^{\infty} \int_{X} \frac{s^{2} t^{2 \kappa}}{\left(s^{2}+t^{2}\right)^{\kappa+1}} K_{\left(s^{2}+t^{2}\right)^{\kappa+1} L^{\kappa+1} e^{-(s+t)} \sqrt{L} F(L)}(y, z) \psi(t \sqrt{L}) f(z) \frac{d t d \mu(z)}{t} \\
& \leq C A \cdot B,
\end{aligned}
$$

where

$$
A^{2}=\int_{0}^{\infty} \int_{X}|\psi(t \sqrt{L}) f(z)|^{2}\left(1+\frac{d(y, z)}{s+t}\right)^{-2 \beta_{0}} \frac{s^{2} t^{2 \kappa}}{\left(s^{2}+t^{2}\right)^{\kappa+1}} \frac{d \mu(z) d t}{t \mu(B(y, s+t))}
$$

and

$$
\begin{aligned}
B^{2} & =\int_{0}^{\infty} \int_{X} \frac{s^{2} t^{2 \kappa}}{\left(s^{2}+t^{2}\right)^{\kappa+1} \mu(B(y, s+t))}\left(1+\frac{d(y, z)}{s+t}\right)^{2 \beta_{0}}\left|K_{\left(s^{2}+t^{2}\right)^{\kappa+1} L^{\kappa+1} e^{-(s+t) \sqrt{L}} F(L)}(y, z)\right|^{2} \frac{d \mu(z) d t}{t} \\
& \leq C \sup _{t>0}\left\|\eta \delta_{t} F\right\|_{W_{\beta}^{q}(\mathbb{R})}^{2},
\end{aligned}
$$

where we used the fact (see [26, Lemma 4.6], [17, Lemma 4.3]) that for any $n / 2<\beta_{0}<\beta$,

$$
\int_{X}\left|\left(1+\frac{d(y, z)}{t}\right)^{\beta_{0}} K_{\left(t^{2} L\right)^{k} e^{-t \sqrt{L}} F(L)}(y, z)\right|^{2} d \mu(z) \leq \frac{C}{\mu(B(y, t))} \sup _{t>0}\left\|\eta \delta_{t} F\right\|_{W_{\beta}^{q}(\mathbb{R})}^{2} .
$$

Therefore, we put it into the definition of $S_{L}$ to obtain

$$
S_{L}^{2}(F(L) f)(x)
$$




$$
\begin{aligned}
= & \int_{0}^{\infty} \int_{d(x, y)<s}\left|s^{2} L e^{-s} \sqrt{L} F(L) f(y)\right|^{2} \frac{d \mu(y) d s}{s \mu(B(x, s))} \\
\leq & C \sup _{t>0}\left\|\eta \delta_{t} F\right\|_{W_{\beta}^{q}(\mathbb{R})}^{2} \int_{0}^{\infty} \int_{X}|\psi(t \sqrt{L}) f(z)|^{2} \\
& \times\left(\int_{0}^{\infty} \int_{d(x, y)<s}\left(1+\frac{d(y, z)}{s+t}\right)^{-\beta_{0}} \frac{s^{2} t^{2 \kappa}}{\left(s^{2}+t^{2}\right)^{\kappa+1}}\left(\frac{\mu(B(x, t))}{\mu(B(x, s)) \mu(B(y, s+t))}\right) \frac{d \mu(y) d s}{s}\right) \frac{d \mu(z) d t}{t \mu(B(x, t))} .
\end{aligned}
$$

We will be done if we show that

$$
\begin{aligned}
& \int_{0}^{\infty} \int_{d(x, y)<s}\left(1+\frac{d(y, z)}{s+t}\right)^{-2 \beta_{0}} \frac{s^{2} t^{2 \kappa}}{\left(s^{2}+t^{2}\right)^{\kappa+1}}\left(\frac{\mu(B(x, t))}{\mu(B(x, s)) \mu(B(y, s+t))}\right) \frac{d \mu(y) d s}{s} \\
\leq & C\left(\frac{t}{t+d(x, z)}\right)^{n \lambda}
\end{aligned}
$$

for some $\lambda>1$. Then we will prove estimate (5.12) by considering the following two cases.

Case (1). $d(x, z) \leq t$. In this case, observe that if $d(x, y) \leq s$, then $\mu(B(x, s+t)) \leq C \mu(B(y, s+t))$. From this, we know that

$$
\text { LHS of }(5.12) \leq C \int_{0}^{\infty} \frac{s^{2} t^{2 \kappa}}{\left(s^{2}+t^{2}\right)^{\kappa+1}} \frac{d s}{s} \leq C .
$$

But $d(x, z) \leq t$, so

$$
\left(\frac{t}{t+d(x, z)}\right)^{n \lambda} \geq C_{n, \lambda}
$$

This implies that (5.12) holds when $d(x, z) \leq t$.

Case (2). $d(x, z)>t$. In this case, we break the integral into two pieces:

$$
\int_{0}^{d(x, z) / 2} \int_{d(x, y)<s} \cdots+\int_{d(x, z) / 2}^{\infty} \int_{d(x, y)<s} \cdots=: I+I I .
$$

For the first term, note that $d(x, y)<s<d(x, z) / 2$, and so $d(y, z) \geq d(x, z)-d(y, x)>d(x, z) / 2$. This yields

$$
\begin{aligned}
I & \leq \int_{0}^{d(x, z) / 2} \int_{d(x, y)<s}\left(1+\frac{d(y, z)}{s+t}\right)^{-2 \beta_{0}} \frac{s^{2} t^{2 \kappa}}{\left(s^{2}+t^{2}\right)^{\kappa+1}}\left(\frac{\mu(B(x, t))}{\mu(B(x, s)) \mu(B(y, s+t))}\right) \frac{d \mu(y) d s}{s} \\
& \leq C \int_{0}^{\infty}\left(\frac{s+t}{d(x, z)}\right)^{2 \beta_{0}} \frac{s^{2} t^{2 \kappa}}{\left(s^{2}+t^{2}\right)^{\kappa+1}} \frac{d s}{s} \\
& \leq C\left(\frac{t}{d(x, z)}\right)^{n \lambda}
\end{aligned}
$$

with $\lambda=2 \beta_{0} / n>1$. For the term $I I$, we have that $s>d(x, z) / 2>t / 2$ and $d(y, z) \leq d(x, y)+d(x, z) \leq$ $3 s$. It yields that

$$
I I \leq C \int_{d(x, z) / 2}^{\infty} \int_{d(x, y)<s}\left(1+\frac{d(y, z)}{s+t}\right)^{-2 \beta_{0}} \frac{s^{2} t^{2 \kappa}}{\left(s^{2}+t^{2}\right)^{\kappa+1}}\left(\frac{\mu(B(x, t))}{\mu(B(x, s)) \mu(B(y, s+t))}\right) \frac{d \mu(y) d s}{s}
$$




$$
\begin{aligned}
& \leq C\left(\frac{t}{d(x, z)}\right)^{2 \kappa} \int_{0}^{\infty} \frac{s^{2}}{\left(s^{2}+t^{2}\right)} \frac{d s}{s} \\
& \leq C\left(\frac{t}{d(x, z)}\right)^{n \lambda}
\end{aligned}
$$

with $\lambda=2 \kappa / n>1$. From the above Cases (1) and (2), we have obtained estimate (5.12), and then the proof of estimate (5.11) is complete.

Proof of Theorem 5.4. Theorem 5.2 and Proposition 5.5, in combination with (5.10), yield that

$$
\begin{aligned}
\|F(L) f\|_{p} \leq C p^{1 / 2}\left\|S_{L}(F(L) f)\right\|_{p} & \leq C p^{1 / 2} \sup _{t>0}\left\|\eta \delta_{t} F\right\|_{W_{\beta}^{q}(\mathbb{R})}\left\|\mathcal{G}_{\lambda}^{*}(f)\right\|_{p} \\
& \leq C p \sup _{t>0}\left\|\eta \delta_{t} F\right\|_{W_{\beta}^{q}(\mathbb{R})}\|f\|_{p}
\end{aligned}
$$

as $p \rightarrow \infty$. This finishes the proof of Theorem 5.4.

Remark. Let $M$ be a complete non-compact connected Riemannian manifold, $\mu$ the Riemannian measure, $\nabla$ the Riemannian gradient. One defines $\Delta$, the Laplace-Beltrami operator, as a self-adjoint positive operator on $L^{2}(M)$. Assume that $M$ satisfies the volume doubling condition (2.1) and the (scaled) Poincare inequalities if there exists $C>0$ such that, for every ball $B=B(x, r)$ and every $f$ with $f, \nabla f$ locally square integrable,

$$
\int_{B}\left|f-f_{B}\right|^{2} d \mu \leq C r^{2} \int_{B}|\nabla f|^{2} d \mu .
$$

It is known (see [2, Theorem 1.3]) that if the gradient of kernel $p_{t}(x, y)$ of the semigroup $e^{-t \Delta}$ satisfies

$$
\left|\nabla p_{t}(x, y)\right| \leq C \frac{C}{\sqrt{t} \mu(B(x, \sqrt{t}))} \exp \left(-c \frac{d^{2}(x, y)}{t}\right), \quad \forall t>0, x, y \in M,
$$

then the Riesz transform $\nabla \Delta^{-1 / 2}$ is bounded on $L^{p}(M)$ for every $1<p<\infty$. Applying the method in the proof of Theorem 5.4, we can show that

$$
\left\|\nabla \Delta^{-1 / 2} f\right\|_{p} \leq C p\|f\|_{p}
$$

as $p \rightarrow \infty$. For the detail, we refer it to [49].

5.3. Two-weighted norm inequalities for the square function. Next we apply Corollary 4.3 to show the following analogous result to Theorem 11.3 in [48].

Theorem 5.6. Assume that $L$ is a densely-defined operator on $L^{2}(X)$ satisfying $(\boldsymbol{H 1}),(\boldsymbol{H} 2)$ and $(\boldsymbol{H 3})$. Let $0<p<\infty, \tau>p / 2$, and let $V$ and $W$ be two weights such that

$$
\int_{Q} V(x)\left(\log \left(e+\frac{V(x)}{V_{Q}}\right)\right)^{\tau} d \mu(x) \leq \int_{Q} W(x) d \mu(x)
$$

for all $Q \in \bigcup_{b=1}^{K} \mathcal{D}^{b}$. Then there exists a positive constant $C=C(p, n, \tau, \rho)$ such that for every $f \in L_{0}^{\infty}(X)$,

$$
\int_{X}|f(x)|^{p} V(x) d \mu(x) \leq C \int_{X}\left(\mathscr{A}_{L}(f)(x)\right)^{p} W(x) d \mu(x) .
$$


As a consequence, for every $\alpha>0$, there exists a positive constant $C=C(p, n, \alpha)$ such that for every $f \in L_{0}^{\infty}(X)$,

$$
\int_{X}|f(x)|^{p} V(x) d \mu(x) \leq C \int_{X}\left(S_{L, \alpha}(f)(x)\right)^{p} W(x) d \mu(x) .
$$

Proof. Let $F_{b, m}(x)=\sum_{E_{2}(Q) \in \bigcup_{-m \leq s \leq m} \mathcal{D}_{s}^{b}} a_{Q}(f)(x)$, it follows from the proof of Theorem 4.4 that there exists $\left\{m_{i}\right\}$ such that

$$
\begin{aligned}
\int_{X}|f(x)|^{p} V(x) d \mu(x) & \leq \lim _{i \rightarrow \infty} \int_{X}\left|\sum_{b=1}^{K} F_{b, m_{i}}(x)\right|^{p} V(x) d \mu(x) \\
& \leq C(K, p) \sum_{b=1}^{K} \frac{\lim }{i \rightarrow \infty} \int_{X}\left|F_{b, m_{i}}(x)\right|^{p} V(x) d \mu(x) .
\end{aligned}
$$

This, together with Corollary 4.3, the condition (5.14) and the similar argument to that of Theorem 11.3 in [48], deduces that for every $0<p<\infty$, there exists a constant $C=C(p, n, \tau, \rho)$ such that

$$
\int_{X}\left|F_{b, m}(x)\right|^{p} V(x) d \mu(x) \leq C \int_{X}\left(\mathscr{A}_{L}\left(F_{b, m}\right)(x)\right)^{p} W(x) d \mu(x)
$$

for every $b \in\{1,2, \cdots, K\}$ and $m>0$.

Notice that $\mathscr{A}_{L}\left(F_{b, m}\right)(x) \leq \mathscr{A}_{L}(f)(x)$, a.e. $x \in X$, arguments above implies that (5.15) holds. This, in combination with Lemma 3.2, deduces (5.16) readily. The proof of Theorem 5.6 is end.

For every $P \in \mathscr{D}$, we denote by $\ell(P)=\delta^{k_{P}}$ where $k_{P}$ is the generational label of $P$ in $\mathscr{D}$, that is, $P \in \mathscr{D}_{k_{P}}$. Corresponding to definitions (4.2) and (3.5), for a given $f \in L^{2}(X)$ and $f_{\mathcal{F}}=\sum_{E_{2}(P) \in \mathcal{F}} a_{P}(f)$ with $\mathcal{F} \subset \mathcal{D}^{b}$ for some $b=1,2, \cdots, K$, define

$$
\widetilde{\mathscr{A}_{L}}\left(f_{\mathcal{F}}\right)(x):=\left(\sum_{P \in \mathscr{D}, E_{2}(P) \in \mathcal{F}} \frac{\left|\lambda_{P}\right|^{2}}{(\ell(P))^{2} \mu(P)} \chi_{E_{2}(P)}(x)\right)^{1 / 2}
$$

and

$$
\widetilde{\mathscr{A}_{L}}(f)(x):=\left(\sum_{P \in \mathscr{D}} \frac{\left|\lambda_{P}\right|^{2}}{(\ell(P))^{2} \mu(P)} \chi_{E_{2}(P)}(x)\right)^{1 / 2} .
$$

The following result is a straightforward result from Corollary 4.3.

Lemma 5.7. Assume that $L$ is a densely-defined operator on $L^{2}(X)$ satisfying (H1), (H2) and (H3). For $f_{\mathcal{G}}$ and $Q \in \mathcal{D}^{b}$ defined in Theorem 4.2. Assume $V$ is a weight, $\int_{Q} V(x) d \mu(x) \neq 0$, and $0<p<\infty$. Then there exists a positive constant $C=C(p, n, \rho)$ independent of $\mathcal{G}, K_{\mathcal{G}}, Q, f$ and $V$ such that

$$
\int_{Q}\left|f_{\mathcal{G}}(x)\right|^{p} V(x) d \mu(x) \leq C(\ell(Q))^{p}\left\|\widetilde{\mathscr{A}_{L}}\left(f_{\mathcal{G}}\right)\right\|_{L^{\infty}(Q)}^{p} \int_{Q} V(x)\left(\log \left(e+\frac{V(x)}{V_{Q}}\right)\right)^{p / 2} d \mu(x) .
$$

Proof. We write

$$
\int_{Q}\left|f_{\mathcal{G}}(x)\right|^{p} V(x) d \mu(x)=(\ell(Q))^{p} \int_{Q}\left|\frac{f_{\mathcal{G}}(x)}{\ell(Q)}\right|^{p} V(x) d \mu(x) .
$$

For every $E_{2}(P) \in \mathcal{G}$ with $P \in \mathscr{D}$, one can apply Proposition 2.2 and $E_{2}(P) \subset Q$ to see $\ell(P) \leq \ell(Q)$. Then the fact $\mathscr{A}_{L}\left(C f_{\mathcal{G}}\right)=|C| \mathscr{A}_{L}\left(f_{\mathcal{G}}\right), C \in \mathbb{R}$, implies that

$$
\mathscr{A}_{L}\left(\frac{f_{\mathcal{G}}(x)}{\ell(Q)}\right)(x)=\left(\sum_{E_{2}(P) \in \mathcal{G}} \frac{\left|\lambda_{P}\right|^{2}}{(\ell(Q))^{2} \mu(P)} \chi_{E_{2}(P)}(x)\right)^{1 / 2}
$$




$$
\begin{aligned}
& \leq\left(\sum_{E_{2}(P) \in \mathcal{G}} \frac{\left|\lambda_{P}\right|^{2}}{(\ell(P))^{2} \mu(P)} \chi_{E_{2}(P)}(x)\right)^{1 / 2} \\
& =\widetilde{\mathscr{A}}_{L}\left(f_{\mathcal{G}}\right)(x) .
\end{aligned}
$$

Then Lemma 5.7 follows from Corollary 4.3.

Next we aim to prove the following two-weighted norm inequalities, which is analogous to Theorems 4.1 and 4.2 in [47]. In the sequel, for every $b \in\{1,2, \cdots, K\}$, similarly as before, for every $Q \in \mathcal{D}^{b}$, we denote by $\ell(Q)=\delta^{k_{Q}}$ where $k_{Q}$ is the generational label of $Q$ in $\mathcal{D}^{b}$, that is, $Q \in \mathcal{D}_{k_{Q}}^{b}$.

Proposition 5.8. Assume that $L$ is a densely-defined operator on $L^{2}(X)$ satisfying (H1), (H2) and (H3). Let $0<p \leq 2, \tau>p / 2$, and let $V$ and $W$ be two weights such that

$$
(\ell(Q))^{p} \int_{Q} V(x)\left(\log \left(e+\frac{V(x)}{V_{Q}}\right)\right)^{\tau} d \mu(x) \leq \int_{Q} W(x) d \mu(x)
$$

for all $Q \in \bigcup_{b=1}^{K} \mathcal{D}^{b}$. There exists a constant $C=C(p, n, \tau, \rho)$, such that for every $f \in L_{0}^{\infty}(X)$,

$$
\int_{X}|f(x)|^{p} V(x) d \mu(x) \leq C \int_{X}\left(\widetilde{\mathscr{A}_{L}}(f)(x)\right)^{p} W(x) d \mu(x) .
$$

We will break up the proof into two cases: $0<p \leq 1$, and $1<p \leq 2$. When $1<p \leq 1$, the proof is based on a modification of the proof of Theorem 11.3 in [48]. When $1<p \leq 2$, the argument is more technical, we shall combine techniques from proofs of [47, Theorem 2.7], [47, Theorem 4.1] and [48, Theorem 11.3], and a crucial ingredient for the proof is Corollary 4.3. In the sequel, for every function $V \in L_{\text {loc }}^{1}(X)$ and set $E$, we write $V(E)=\int_{E} V(x) d \mu(x)$.

Proof of Proposition 5.8 for $0<p \leq 1$. For every $b \in\{1,2, \cdots, K\}$ and $m \geq 1$, define $F_{b, m}=$ $\sum_{E_{2}(P) \in \cup_{-m \leq s \leq m} \mathcal{D}_{s}^{b}} a_{P}(f)(x)$ as we did in the proof of Theorem 5.6. With some notations as in the proof of Theorem 5.1, for $j \in \mathbb{Z}$ we set

$$
\begin{gathered}
\widetilde{E}_{b, m}^{j}=\left\{x \in X: \widetilde{\mathscr{A}}_{L}\left(F_{b, m}\right)(x)>2^{j}\right\}, \\
\widetilde{D}_{b, m}^{j}=\left\{E_{2}(Q): Q \in \mathscr{D}, E_{2}(Q) \in \bigcup_{-m \leq s \leq m} \mathcal{D}_{s}^{b}, E_{2}(Q) \subset \widetilde{E}_{b, m}^{j}, E_{2}(Q) \not \subset \widetilde{E}_{b, m}^{j+1}\right\}, \\
\widetilde{F}_{b, m}^{j}(x)=\sum_{E_{2}(Q) \in \widetilde{D}_{b, m}^{j}} a_{Q}(f)(x), \quad j \in \mathbb{Z},
\end{gathered}
$$

and

$$
\widetilde{\mathcal{B}}_{b, m}^{j}=\left\{Q_{b, m}^{j}: Q_{b, m}^{j} \text { is the maximal dyadic cube in } \widetilde{D}_{b, m}^{j}\right\} .
$$

From the discussion in the proof of Theorem 5.1, we have the following conclusions:

(1) Sets $\widetilde{D}_{b, m}^{j}$ for different $j$ are disjoint (some of them might be empty) and $\bigcup_{-m \leq s \leq m} \mathcal{D}_{s}^{b}=\cup_{j} \widetilde{D}_{b, m}^{j}$.

(2) $F_{b, m}=\sum_{j} \widetilde{F}_{b, m}^{j}$.

(3) $\widetilde{\mathscr{A}_{L}}\left(F_{b, m}^{j}\right) \leq 2^{j+1}$ everywhere.

(4) Any two cubes in $\widetilde{\mathcal{B}}_{b, m}^{j}$ are disjoint. Besides it is easy to check

$$
\operatorname{supp} \widetilde{F}_{b, m}^{j} \subset \bigcup_{Q_{b, m}^{j} \in \widetilde{\mathcal{B}}_{b, m}^{j}} Q_{b, m}^{j} \subset \widetilde{E}_{b, m}^{j} .
$$


Without loss of generality, we assume that $V_{Q_{b, m}^{j}} \neq 0$ for all $Q_{b, m}^{j} \in \widetilde{\mathcal{B}}_{b, m}^{j}$. For $0<p \leq 1$,

$$
\begin{aligned}
\int_{X}\left|F_{b, m}(x)\right|^{p} V(x) d \mu(x) & \leq \sum_{j \in \mathbb{Z}} \sum_{Q_{b, m}^{j} \in \widetilde{\mathcal{B}}_{b, m}^{j}} \int_{Q_{b, m}^{j}}\left|\widetilde{F}_{b, m}^{j}(x)\right|^{p} V(x) d \mu(x) \\
& \leq \sum_{j \in \mathbb{Z}} \sum_{Q_{b, m}^{j} \in \widetilde{\mathcal{B}}_{b, m}^{j}}\left(V\left(Q_{b, m}^{j}\right)\right)^{1-p /(2 \tau)}\left(\int_{Q_{b, m}^{j}}\left|\widetilde{F}_{b, m}^{j}(x)\right|^{2 \tau} V(x) d \mu(x)\right)^{p /(2 \tau)} .
\end{aligned}
$$

Notice that $\left\|\widetilde{\mathscr{A}_{L}}\left(F_{b, m}^{j}\right)\right\|_{L^{\infty}} \leq 2^{j+1}$, one can apply Lemma 5.7 and (5.20) to see

$$
\begin{aligned}
\int_{Q_{b, m}^{j}}\left|\widetilde{F}_{b, m}^{j}(x)\right|^{2 \tau} V(x) d \mu(x) & \leq C 2^{2 \tau j}\left(\ell\left(Q_{b, m}^{j}\right)\right)^{2 \tau} \int_{Q_{b, m}^{j}} V(x)\left(\log \left(e+\frac{V(x)}{V_{Q_{b, m}^{j}}}\right)\right)^{\tau} d \mu(x) \\
& \leq C 2^{2 \tau j}\left(\ell\left(Q_{b, m}^{j}\right)\right)^{2 \tau-p} W\left(Q_{b, m}^{j}\right) .
\end{aligned}
$$

Put it into (5.22), we obtain

$$
\begin{aligned}
\int_{X}\left|F_{b, m}(x)\right|^{p} V(x) d \mu(x) & \leq C \sum_{j \in \mathbb{Z}} 2^{p j} \sum_{Q_{b, m}^{j} \in \widetilde{\mathcal{B}}_{b, m}^{j}}\left[\left[\ell\left(Q_{b, m}^{j}\right)\right]^{p} V\left(Q_{b, m}^{j}\right)\right]^{1-p /(2 \tau)}\left(W\left(Q_{b, m}^{j}\right)\right)^{p /(2 \tau)} \\
& \leq C \sum_{j \in \mathbb{Z}} 2^{p j} \sum_{Q_{b, m}^{j} \in \widetilde{\mathcal{B}}_{b, m}^{j}} W\left(Q_{b, m}^{j}\right) \\
& =C \sum_{j \in \mathbb{Z}} 2^{p j} \sum_{Q_{b, m}^{j} \in \widetilde{\mathcal{B}}_{b, m}^{j}} \int_{Q_{b, m}^{j}} W(x) d \mu(x) \\
& =C \sum_{j \in \mathbb{Z}} \sum_{Q_{b, m}^{j} \in \widetilde{\mathcal{B}}_{b, m}^{j}} \int_{Q_{b, m}^{j}}\left(\widetilde{\mathscr{A}_{L}}\left(F_{b, m}\right)(x)\right)^{p} W(x) d \mu(x) \\
& \leq C \int_{X}\left(\widetilde{\mathscr{A}_{L}}\left(F_{b, m}\right)(x)\right)^{p} W(x) d \mu(x)
\end{aligned}
$$

where the second inequality follows from the fact that (5.20) implies $\left[\ell\left(Q_{b, m}^{j}\right)\right]^{p} V\left(Q_{b, m}^{j}\right) \leq W\left(Q_{b, m}^{j}\right)$.

This, together with (5.17), implies that Proposition 5.8 holds for $0<p \leq 1$.

To show Proposition 5.8 for $1<p \leq 2$, we need the following lemma.

Lemma 5.9. Let $0<p<\infty, \tau>p / 2$, and let $V$ be a weight. Assume $\mathcal{F} \subset \mathcal{D}^{b}$ for some $b \in$ $\{1,2, \cdots, K\}, \# \mathcal{F}<\infty$ and there exists some $k \in \mathbb{N}$ such that

$$
2^{k} \leq \frac{1}{\int_{Q} V(x) d \mu(x)} \int_{Q} V(x)\left(\log \left(e+\frac{V(x)}{V_{Q}}\right)\right)^{\tau} d \mu(x)<2^{k+1}, \quad \forall Q \in \mathcal{F} .
$$

For any $f \in L_{0}^{\infty}(X)$, define $f_{\mathcal{F}}=\sum_{E_{2}(P) \in \mathcal{F}} a_{P}(f)$. Then there exists a positive constant $C(p, n, \tau, \rho)$ independent of $\mathcal{F}$ and $f$, such that

$$
\int_{X}\left|f_{\mathcal{F}}(x)\right|^{p} V(x) d \mu(x) \leq C 2^{[p /(2 \tau)] k} \int_{X}\left|\mathscr{A}_{L}\left(f_{\mathcal{F}}\right)(x)\right|^{p} V(x) d \mu(x) .
$$

Proof. Similar to notations in the proof of Proposition 5.1, set

$$
E_{j}=\left\{x \in X: \mathscr{A}_{L}\left(f_{\mathcal{F}}\right)(x)>2^{j}\right\},
$$




$$
\begin{gathered}
D_{j}=\left\{E_{2}(Q) \in \mathcal{F}: \quad E_{2}(Q) \subset E_{j}, E_{2}(Q) \not \subset E_{j+1}\right\}, j \in \mathbb{Z}, \\
f_{\mathcal{F}, j}(x)=\sum_{E_{2}(Q) \in D_{j}} a_{Q}(f)(x), \quad j \in \mathbb{Z},
\end{gathered}
$$

and

$$
\mathcal{B}_{j}=\left\{E_{2}(Q): E_{2}(Q) \text { is the maximal dyadic cube in } D_{j}\right\} .
$$

A key observation is that using a similar argument to that in [48, pp. 192], we obtain $f_{\mathcal{F}}(x)=$ $\sum_{i: i \leq j} f_{\mathcal{F}, i}(x)$ when $x \in E_{j} \backslash E_{j+1}$.

Let $\varepsilon>0$ to be chosen later. Recall that $\left\{x \in X: f_{\mathcal{F}}(x) \neq 0\right\} \subset \bigcup_{j} E_{j}$, we follow [48, (11.4)] to see

$$
\begin{aligned}
\int_{X}\left|f_{\mathcal{F}}(x)\right|^{p} V(x) d \mu(x) & =\sum_{j \in \mathbb{Z}} \int_{E_{j} \backslash E_{j+1}}\left|f_{\mathcal{F}}(x)\right|^{p} V(x) d \mu(x) \\
& =\sum_{j \in \mathbb{Z}} \int_{E_{j} \backslash E_{j+1}}\left|\sum_{i: i \leq j} f_{\mathcal{F}, i}(x)\right|^{p} V(x) d \mu(x) \\
& \leq C \sum_{j \in \mathbb{Z}} \int_{E_{j} \backslash E_{j+1}} \sum_{i: i \leq j} 2^{\varepsilon(j-i)}\left|f_{\mathcal{F}, i}(x)\right|^{p} V(x) d \mu(x) \\
& \leq C \sum_{i, j: i \leq j} 2^{\varepsilon(j-i)} \int_{E_{j}}\left|f_{\mathcal{F}, i}(x)\right|^{p} V(x) d \mu(x) \\
& \leq C \sum_{i, j: i \leq j} 2^{\varepsilon(j-i)}\left(V\left(E_{j}\right)\right)^{1-p /(2 \tau)}\left(\int_{E_{i}}\left|f_{\mathcal{F}, i}(x)\right|^{2 \tau} V(x) d \mu(x)\right)^{p /(2 \tau)},
\end{aligned}
$$

where the last inequality follows from Hölder's inequality and $E_{j} \subset E_{i}$ for $i \leq j$.

Notice that $Q_{i} \in \mathcal{F}$ for every $Q_{i} \in \mathcal{B}_{i}$. It follows from Corollary 4.3 and (5.24) that

$$
\begin{aligned}
\int_{E_{i}}\left|f_{\mathcal{F}, k}(x)\right|^{2 \tau} V(x) d \mu(x) & =\sum_{Q_{i} \in \mathcal{B}_{i}} \int_{Q_{i}}\left|f_{\mathcal{F}, i}(x)\right|^{2 \tau} V(x) d \mu(x) \\
& \leq C 2^{2 i \tau} \sum_{Q_{i} \in \mathcal{B}_{i}} \int_{Q_{i}} V(x)\left(\log \left(e+\frac{V(x)}{V_{Q_{i}}}\right)\right)^{\tau} d \mu(x) \\
& \leq C 2^{2 i \tau} 2^{k} \sum_{Q_{i} \in \mathcal{B}_{i}} V\left(Q_{i}\right) \\
& \leq C 2^{2 i \tau} 2^{k} V\left(E_{k}\right) .
\end{aligned}
$$

Put it into (5.26), and we follow estimates in [48, (11.5)] to obtain

$$
\begin{aligned}
\int_{X}\left|f_{\mathcal{F}}(x)\right|^{p} V(x) d \mu(x) \leq & C \sum_{i, j: i \leq j} 2^{\varepsilon(j-i)}\left(V\left(E_{j}\right)\right)^{1-p /(2 \tau)} 2^{i p} 2^{p k /(2 \tau)}\left(V\left(E_{k}\right)\right)^{p /(2 \tau)} \\
\leq & C 2^{p k /(2 \tau)} \sum_{i, j: i \leq j} 2^{-\{p[1-p /(2 \tau)]-\varepsilon\}(j-i)}\left[2^{j p} V\left(E_{j}\right)\right]^{1-p /(2 \tau)}\left[2^{i p} V\left(E_{k}\right)\right]^{p /(2 \tau)} \\
\leq & C 2^{p k /(2 \tau)} \sum_{i, j: i \leq j} 2^{-\{p[1-p /(2 \tau)]-\varepsilon\}(j-i)}\left[(1-p /(2 \tau)) 2^{j p} V\left(E_{j}\right)\right] \\
& +C 2^{p k /(2 \tau)} \sum_{i, j: i \leq j} 2^{-\{p[1-p /(2 \tau)]-\varepsilon\}(j-i)}\left[(p /(2 \tau)) 2^{k p} V\left(E_{i}\right)\right]
\end{aligned}
$$


Choose $\varepsilon=\frac{p[1-p /(2 \tau)]}{2}>0$, one can apply the last part in the proof of [48, Theorem 11.3] to obtain $(5.25)$ readily and we omit it.

Proof of Proposition 5.8 for $1<p \leq 2$. For every $b \in\{1,2, \cdots, K\}$ and $m \geq 1$, define $F_{b, m}=$ $\sum_{E_{2}(P) \in \cup_{-m \leq s \leq m} \mathcal{D}_{s}^{b}} a_{P}(f)(x)$. Following the line of [47, Theorem 2.7], for every $k \in \mathbb{N}$, define

$$
\mathcal{G}_{b, m}^{k}=\left\{E_{2}(P) \in \bigcup_{-m \leq s \leq m} \mathcal{D}_{s}^{b}: 2^{k} \leq \frac{1}{\int_{E_{2}(P)} V(x) d \mu(x)} \int_{E_{2}(P)} V(x)\left(\log \left(e+\frac{V(x)}{V_{E_{2}(P)}}\right)\right)^{\tau} d \mu(x)<2^{k+1}\right\},
$$

and

$$
G_{b, m}^{k}(x)=\sum_{E_{2}(P) \in \mathcal{G}_{b, m}^{k}} a_{P}(f)(x) .
$$

It's cleat that $\mathcal{G}_{b, m}^{i} \cap \mathcal{G}_{b, m}^{j}=\emptyset$ for arbitrary $i \neq j$, and

$$
\bigcup_{-m \leq s \leq m} \mathcal{D}_{s}^{b}=\bigcup_{k \in \mathbb{N}} \mathcal{G}_{b, m}^{k}
$$

For every $k \in \mathbb{N}$, define

$$
\mathcal{E}_{E_{2}(R)}\left(G_{b, m}^{k}\right) \equiv\left(\sum_{E_{2}(R) \subset E_{2}(P) \in \mathcal{G}_{b, m}^{k}} \frac{\left|\lambda_{R}\right|^{2}}{\mu(R)}\right)^{p / 2}-\left(\sum_{E_{2}(R) \subsetneq E_{2}(P) \in \mathcal{G}_{b, m}^{k}} \frac{\left|\lambda_{R}\right|^{2}}{\mu(R)}\right)^{p / 2}
$$

for every $E_{2}(R) \in \bigcup_{-m \leq s \leq m} \mathcal{D}_{s}^{b}$. As shown in the proof of [47, Theorem 2.7],

$$
\begin{gathered}
F_{b, m}=\sum_{i \in \mathbb{N}} G_{b, m}^{k}, \\
\mathcal{E}_{E_{2}(R)}\left(G_{b, m}^{k}\right)=0 \text { if } E_{2}(R) \notin \mathcal{G}_{b, m}^{k},
\end{gathered}
$$

and

$$
\left(\mathscr{A}_{L}\left(G_{b, m}^{k}\right)\right)^{p}(x)=\sum_{x \in E_{2}(R) \in \mathcal{G}_{b, m}^{i}} \mathcal{E}_{E_{2}(R)}\left(G_{b, m}^{k}\right) .
$$

The left of the proof is to follow the technique as in the proof of [47, Theorem 2.7] and [47, Theorem 4.1]. In details, for every $E_{2}(P) \in \mathcal{G}_{b, m}^{k}, k \in \mathbb{N}$, it follows from the definition of $\mathcal{G}_{b, m}^{k}$ and (5.20) to see

$$
2^{k} V\left(E_{2}(P)\right) \leq \int_{E_{2}(P)} V(x)\left(\log \left(e+\frac{V(x)}{V_{E_{2}(P)}}\right)\right)^{\tau} d \mu(x) \leq \frac{W\left(E_{2}(P)\right)}{\left[\ell\left(E_{2}(P)\right)\right]^{p}},
$$

and the proof of [47, Theorem 4.1] shows that since $p / 2 \leq 1$,

$$
\begin{aligned}
\frac{\mathcal{E}_{E_{2}(P)}\left(G_{b, m}^{k}\right)}{\left[\ell\left(E_{2}(P)\right)\right]^{p}} & \leq\left(\sum_{E_{2}(R) \subset E_{2}(P) \in \mathcal{G}_{b, m}^{k}} \frac{\left|\lambda_{R}\right|^{2}}{(\ell(R))^{2} \mu(R)}\right)^{p / 2}-\left(\sum_{E_{2}(R) \varsubsetneqq E_{2}(P) \in \mathcal{G}_{b, m}^{k}} \frac{\left|\lambda_{R}\right|^{2}}{(\ell(R))^{2} \mu(R)}\right)^{p / 2} \\
& =: \widetilde{\mathcal{E}}_{E_{2}(P)}\left(G_{b, m}^{k}\right) .
\end{aligned}
$$

Moreover,

$$
\left(\widetilde{\mathscr{A}_{L}}\left(G_{b, m}^{k}\right)\right)^{p}(x)=\sum_{x \in E_{2}(P) \in \mathcal{G}_{b, m}^{k}} \widetilde{\mathcal{E}}_{E_{2}(P)}\left(G_{b, m}^{k}\right)
$$


Observe that for any increasing function $\boldsymbol{\aleph}:[0, \infty] \rightarrow[1, \infty)$ satisfying $\sum_{k=0}^{\infty} \boldsymbol{\aleph}(k)^{-1 /(p-1)} \leq 1$, there exists a constant $C=C(\boldsymbol{\aleph})$ such that

$$
\int_{X}\left|F_{b, m}(x)\right|^{p} V(x) d \mu(x) \leq C(\boldsymbol{\aleph}) \sum_{k \in \mathbb{N}} \boldsymbol{\aleph}(k) \int_{X}\left|G_{b, m}^{k}(x)\right|^{p} V(x) d \mu(x) .
$$

Since $f \in L_{0}^{\infty}(X)$, we have $\# \mathcal{G}_{b, m}^{k}<\infty$ for every $k \in \mathbb{N}$. Hence one can apply Lemma 5.9 and take $\boldsymbol{\aleph}(k)=2^{[1 / 2-p /(4 \tau)] k}, k \in \mathbb{N}$, to obtain when $1<p \leq 2$,

$$
\begin{aligned}
\int_{X}\left|F_{b, m}(x)\right|^{p} V(x) d \mu(x) & \leq C \sum_{k \in \mathbb{N}} 2^{[1 / 2-p /(4 \tau)] k} \int_{X}\left|G_{b, m}^{k}(x)\right|^{p} V(x) d \mu(x) \\
& \leq C \sum_{k \in \mathbb{N}} 2^{[1 / 2-p /(4 \tau)] k} 2^{[p /(2 \tau)] k} \int_{X}\left|\mathscr{A}_{L}\left(G_{b, m}^{k}\right)(x)\right|^{p} V(x) d \mu(x) \\
& =C \sum_{k \in \mathbb{N}} 2^{-[1 / 2-p /(4 \tau)] k} 2^{k} \sum_{E_{2}(R) \in \mathcal{G}_{b, m}^{k}} \mathcal{E}_{E_{2}(R)}\left(G_{b, m}^{k}\right) V\left(E_{2}(R)\right) \\
& \leq C \sum_{k \in \mathbb{N}} 2^{-[1 / 2-p /(4 \tau)] k} \sum_{E_{2}(R) \in \mathcal{G}_{b, m}^{k}} \frac{\mathcal{E}_{E_{2}(R)}\left(G_{b, m}^{k}\right)}{\left[\ell\left(E_{2}(R)\right)\right]^{p}} W\left(E_{2}(R)\right) \\
& \leq C \sum_{k \in \mathbb{N}} 2^{-[1 / 2-p /(4 \tau)] k} \sum_{E_{2}(R) \in \mathcal{G}_{b, m}^{k}} \widetilde{\mathcal{E}}_{E_{2}(R)}\left(G_{b, m}^{k}\right) W\left(E_{2}(R)\right) \\
& \leq C \int_{X}\left(\widetilde{\mathscr{A}}_{L}\left(F_{b, m}\right)\right)^{p}(x) W(x) d \mu(x),
\end{aligned}
$$

where the last inequality follows from $1 / 2-p /(4 \tau)>0$.

This, together with (5.17), implies that (5.21) holds for $1<p \leq 2$. The proof of Proposition 5.8 is complete.

As a consequence of Proposition 5.8, we have the following result.

Theorem 5.10. Assume that $L$ is a densely-defined operator on $L^{2}(X)$ satisfying $(\boldsymbol{H 1}),(\boldsymbol{H} 2)$ and (H3). Let $0<p \leq 2, \tau>p / 2$, and let $V$ and $W$ be two weights such that

$$
(\ell(Q))^{p} \int_{Q} V(x)\left(\log \left(e+\frac{V(x)}{V_{Q}}\right)\right)^{\tau} d \mu(x) \leq \int_{Q} W(x) d \mu(x)
$$

for all $Q \in \bigcup_{b=1}^{K} \mathcal{D}^{b}$. For every $\alpha>0$, there exists a positive constant $C=C(p, n, \tau, \alpha)$, such that for every $f \in L_{0}^{\infty}(X)$,

$$
\int_{X}|f(x)|^{p} V(x) d \mu(x) \leq C \int_{X}\left(\widetilde{S}_{L, \alpha}(\sqrt{L} f)(x)\right)^{p} W(x) d \mu(x) .
$$

where

$$
\widetilde{S}_{L, \alpha}(g)(x):=\left(\int_{0}^{\infty} \int_{d(y, x)<\alpha t}\left|t \sqrt{L} e^{-t \sqrt{L}} g(y)\right|^{2} \frac{d y}{\mu(B(x, t))} \frac{d t}{t}\right)^{1 / 2}, g \in L^{2}(X), \quad \alpha>0 .
$$

In particular, when $1<p \leq 2$ and $W \in A_{p}$, there exists a positive constant $C=C\left(p, n, \tau,\|W\|_{A_{p}}\right)$ such that for every $f \in L_{0}^{\infty}(X)$,

$$
\int_{X}|f(x)|^{p} V(x) d \mu(x) \leq C \int_{X}|\sqrt{L} f(x)|^{p} W(x) d \mu(x) .
$$


Proof. Similar to Lemma 3.2, one can verify that there exists a constant $C=C(n, \alpha)>0$ such that

$$
\widetilde{\mathscr{A}}_{L}(f)(x) \leq C \widetilde{S}_{L, \alpha}(\sqrt{L} f)(x) \text {, a.e. } x \in X \text {. }
$$

The proof of (5.28) is similar to that of Theorem 5.2 and we omit it. Furthermore, one can apply [25, Proposition 3.3] and [26, Proposition 3.4] to deduce that $\widetilde{S}_{L, 1}$ is bounded on $L_{w}^{q}(X)$ for $w \in A_{q}$, $1<q<\infty$. Hence, when $1<p \leq 2$ and $W \in A_{p}$,

$$
\int_{X}\left(\widetilde{S}_{L, 1}(\sqrt{L} f)(x)\right)^{p} W(x) d \mu(x) \leq C \int_{X}|\sqrt{L} f(x)|^{p} W(x) d \mu(x)
$$

for some constant $C=C\left(p, n,\|W\|_{A_{p}}\right)$, which deduces that (5.29) holds. The proof of Theorem 5.10 is finished.

\section{EIGENVALUE ESTIMATES FOR SchröDINGER OPERATORS}

Recall that $\Omega=\left\{(X, y) \in \mathbb{R}^{n-1} \times \mathbb{R}: y>\Phi(X)\right\}$ is a special Lipschitz domain of $\mathbb{R}^{n}$ where $\Phi: \mathbb{R}^{n-1} \mapsto \mathbb{R}$ is a Lipschitz function, i.e., $\|\nabla \Phi\|_{\infty} \leq M<\infty$ for some constant $M$. Let $A(x)$ be an $n \times n$ matrix function with real symmetric, bounded measurable entries on $\mathbb{R}^{n}$ satisfying the ellipticity condition

$$
\|A\|_{\infty} \leq \lambda^{-1} \text { and } A(x) \xi \cdot \xi \geq \lambda|\xi|^{2}
$$

for some constant $\lambda \in(0,1)$, for all $\xi \in \mathbb{R}^{n}$ and for almost all $x \in \mathbb{R}^{n}$. Let $V \in L_{\text {loc }}^{1}(\Omega)$ be nonnegative. Let $H$ be a closed subspace of

$$
\left\{u \in W^{1,2}(\Omega): \int_{\Omega} V|u|^{2} d x<\infty\right\}
$$

containing $W_{0}^{1,2}(\Omega)$, and $\mathcal{L}$ be a second order elliptic operator in divergence form $\mathcal{L}=-\operatorname{div}(A \nabla)-V$ on $L^{2}(\Omega)$ with largest domain $\operatorname{Dom}(\mathcal{L}) \subset H$ such that

$$
\langle\mathcal{L} f, g\rangle=\int_{\Omega} A \nabla f \cdot \nabla g d x-\int_{\Omega} V f \cdot g d x, \quad \forall f \in \operatorname{Dom}(\mathcal{L}), \quad \forall g \in H .
$$

We use the notation $\mathcal{L}=(A, \Omega, H)$ to denote any operator defined as above. We say that $\mathcal{L}$ satisfies the Dirichlet boundary condition (DBC) when $H=W_{0}^{1,2}(\Omega)$, the Neumann boundary condition (NBC) when $H=W^{1,2}(\Omega)$.

In the sequel, we denote $\mathcal{L}$ by $L$ when $V=0$. It is known that when $\Omega=\mathbb{R}^{n}$ or $\Omega$ be a special Lipschitz domain of $\mathbb{R}^{n}$ and $L=-\operatorname{div}(A \nabla)$ is real symmetric operators (under DBC or NBC) as above, properties (H1), (H2) and (H3) for operator $L$ are always satisfied (see [4, 15]). Furthermore, it follows by (6.1) that there exists a constant $C>0$ such that for every $f \in L^{2}(\Omega)$

$$
\lambda\|\nabla f\|_{2} \leq\left\|L^{1 / 2} f\right\|_{2} \leq \lambda^{-1}\|\nabla f\|_{2} .
$$

Now, let $\mathcal{L}=(A, \Omega, V)$ be as above. In studying the spectrum of $\mathcal{L}$, a central question is to find conditions for $\mathcal{L} \geq 0$ (see [9, 8,21,31]). Observe that integration by parts, together with (6.2), shows that $\mathcal{L} \geq 0$ follows from the inequality

$$
\int_{\Omega}|f(x)|^{2} V(x) d x \leq c \int_{\Omega}|\nabla f(x)|^{2} d x
$$

for all $f \in \operatorname{Dom}(\mathcal{L})$ and $c>0$ sufficiently small.

To show (6.5), we apply the previous result in the setting $X=\Omega$ and $L$ to give a sufficient condition for $\mathcal{L} \geq 0$, by adapting the argument as in Theorem 5.1 in [47] to the present situation. 
Theorem 6.1. When $\Omega=\mathbb{R}^{n}$ or $\Omega$ be a special Lipschitz domain of $\mathbb{R}^{n}$ and $\mathcal{L}=-\operatorname{div}(A \nabla)-V$ be real symmetric operators (under $D B C$ or $N B C$ ) with nonnegative $V \in L_{\text {loc }}^{1}(\Omega)$ as above. Let $\sigma(\mathcal{L})$ be the lowest nonpositive eigenvalue of $\mathcal{L}$ on $\Omega$. Fix a $\tau>1$. For every ball $B=B\left(x_{B}, r_{B}\right)$ in $\Omega$,

$$
\Lambda(B, V)=\int_{B} V(x)\left(\log \left(e+\frac{V(x)}{V_{B}}\right)\right)^{\tau} d \mu(x) .
$$

There exist two positive constants $c_{1}, c_{2}$ which depend on $n, \tau$ such that

$$
\sigma(\mathcal{L}) \geq-\sup _{B} c_{1}\left[|B|^{-1} \Lambda(B, V)-c_{2} r_{B}^{-2}|B|\right],
$$

where $|B|$ denotes the Lebesgue measure of $B$ in $\Omega$.

As a consequence, if

$$
r_{B}^{2} \Lambda(B, V) \leq c_{2}|B|
$$

for all balls $B$, then $\mathcal{L} \geq 0$.

To show Theorem 6.1, for every $Q \in \mathcal{D}=\bigcup_{b=1}^{K} \mathcal{D}^{b}$ we define

$$
\Lambda(Q, V)=\int_{Q} V(x)\left(\log \left(e+\frac{V(x)}{V_{Q}}\right)\right)^{\tau} d \mu(x), \quad \tau>1
$$

Let $\ell(Q)$ denote the side length of the cube $Q$. Then the following result holds.

Lemma 6.2. Fix $\tau>1$. For every $Q \in \mathcal{D}$, there exists ball $B$ such that $Q \subset B$ and $|B| \leq C|Q|$ and $r_{B} \sim \ell(Q)$ such that

$$
\Lambda(Q, V) \leq C \Lambda(B, V) .
$$

Proof. Recall that from [48, Theorem 11.2], if $V_{Q} \neq 0$, for all $\gamma>0$,

$$
\int_{Q} V(x)\left(\log \left(C_{6}+\frac{V(x)}{V_{Q}}\right)\right)^{\gamma} d \mu(x) \sim \sup \left\{\int_{Q} V(x) \phi(x) d \mu(x): \phi \in \operatorname{Exp}_{e}(Q, 1 / \gamma)\right\},
$$

where $C_{6}$ is the constant in (4.15), and

$$
\operatorname{Exp}_{e}(Q, 1 / \gamma)=\left\{\psi: \frac{1}{|Q|} \int_{Q} \exp \left(\psi^{1 / \gamma}(x)\right) d \mu(x) \leq e+1\right\} .
$$

Note that (6.10) still holds if we replace dyadic cube $Q$ by ball $B$.

For $\phi \in \operatorname{Exp}_{e}(Q, 1 / \tau)$, define $\tilde{\phi}(x)=\phi(x)$ for $x \in Q$ and $\tilde{\phi}(x)=0$ for $x \notin Q$. Then

$$
\begin{aligned}
\frac{1}{|B|} \int_{B} \exp \left(\tilde{\phi}^{1 / \tau}(x)\right) d \mu(x) & =\frac{1}{|B|} \int_{Q} \exp \left(\tilde{\phi}^{1 / \tau}(x)\right) d \mu(x)+\frac{1}{|B|} \int_{B \backslash Q} \exp \left(\tilde{\phi}^{1 / \tau}(x)\right) d \mu(x) \\
& \leq \frac{1}{|Q|} \int_{Q} \exp \left(\phi^{1 / \tau}(x)\right) d \mu(x)+1 \\
& \leq e+1+1,
\end{aligned}
$$

which implies that $\tilde{\phi} \in \operatorname{Exp}_{e+1}(B, 1 / \tau)$. Then for all $\phi \in \operatorname{Exp}_{e}(Q, 1 / \tau)$

$$
\begin{aligned}
\int_{Q} V(x) \phi(x) d \mu(x) & =\int_{B} V(x) \tilde{\phi}(x) d \mu(x) \\
& \leq \sup \left\{\int_{B} V(x) \psi(x) d \mu(x): \psi \in \operatorname{Exp}_{e+1}(B, 1 / \tau)\right\} .
\end{aligned}
$$


Therefore,

$$
\begin{aligned}
\Lambda(Q, V) & =\int_{Q} V(x)\left(\log \left(e+\frac{V(x)}{V_{Q}}\right)\right)^{\tau} d \mu(x) \\
& \leq C \sup \left\{\int_{Q} V(x) \psi(x) d \mu(x): \psi \in \operatorname{Exp}_{e}(Q, 1 / \tau)\right\} \\
& \leq C \sup \left\{\int_{B} V(x) \psi(x) d \mu(x): \psi \in \operatorname{Exp}_{e+1}(B, 1 / \tau)\right\} \\
& \leq C \int_{B} V(x)\left(\log \left(e+1+\frac{V(x)}{V_{B}}\right)\right)^{\tau} d \mu(x) \\
& \leq C \int_{B} V(x)\left(\log \left(e+\frac{V(x)}{V_{B}}\right)\right)^{\tau} d \mu(x) \\
& \leq C \Lambda(B, V) .
\end{aligned}
$$

This proves Lemma 6.2.

Proof of Theorem 6.1. First, we assume that for all $Q \in \mathcal{D}$,

$$
|Q|^{-1} \Lambda(Q, V)-c_{3}(\ell(Q))^{-2}|Q| \leq C_{11},
$$

where $c_{3}$ will be chosen later. We will show that $\mathcal{L} \geq-c C_{11}$ for some positive constant $c$.

For arbitrary $f \in C_{0}^{\infty}(\Omega)$, let $F_{b, m}, \mathcal{G}_{b, m}^{k}, G_{b, m}^{k}, \mathcal{E}_{E_{2}(P)}\left(G_{b, m}^{k}\right), \widetilde{\mathcal{E}}_{E_{2}(P)}\left(G_{b, m}^{k}\right)$ be as in the proof of Proposition 5.8 for $1<p \leq 2$, with $X=\Omega$ and the corresponding self-adjoint operator is $L=$ $-\operatorname{div}(A \nabla)$. It follows from the proof of Theorem 4.4 that there exists $\left\{m_{i}\right\}$ such that

$$
\begin{aligned}
\int_{X}|f(x)|^{p} V(x) d \mu(x) & \leq \lim _{i \rightarrow \infty} \int_{X}\left|\sum_{b=1}^{K} F_{b, m_{i}}(x)\right|^{p} V(x) d \mu(x) \\
& \leq C(K, p) \sum_{b=1}^{K} \frac{\lim }{i \rightarrow \infty} \int_{X}\left|F_{b, m_{i}}(x)\right|^{p} V(x) d \mu(x) .
\end{aligned}
$$

An argument as in (5.27) shows that

$$
\begin{aligned}
\int_{X}\left|F_{b, m}(x)\right|^{p} V(x) d \mu(x) & \leq C \sum_{k \in \mathbb{N}} 2^{k} \sum_{Q \in \mathcal{G}_{b, m}^{k}} \mathcal{E}_{Q}\left(G_{b, m}^{k}\right) V(Q) \\
& \leq C \sum_{k \in \mathbb{N}} \sum_{Q \in \mathcal{G}_{b, m}^{k}} \mathcal{E}_{Q}\left(G_{b, m}^{k}\right) \Lambda(Q, V) \\
& \leq C \sum_{k \in \mathbb{N}} \sum_{Q \in \mathcal{G}_{b, m}^{k}}\left[c_{3} \frac{\mathcal{E}_{Q}\left(G_{b, m}^{k}\right)}{(\ell(Q))^{2}}|Q|+C_{11} \mathcal{E}_{Q}\left(G_{b, m}^{k}\right)|Q|\right] \\
& \leq C \sum_{k \in \mathbb{N}} \sum_{Q \in \mathcal{G}_{b, m}^{k}}\left[c_{3} \widetilde{\mathcal{E}}_{Q}\left(G_{b, m}^{k}\right)|Q|+C_{11} \mathcal{E}_{Q}\left(G_{b, m}^{k}\right)|Q|\right] \\
& =C \sum_{k \in \mathbb{N}}\left\{c_{3} \int_{\Omega}\left(\widetilde{\mathscr{A}}_{L}\left(G_{b, m}^{k}\right)\right)^{2}(x) d x+C_{11} \int_{\Omega}\left(\mathscr{A}_{L}\left(G_{b, m}^{k}\right)\right)^{2}(x) d x\right\} \\
& =C\left\{c_{3} \int_{\Omega}\left(\widetilde{\mathscr{A}}_{L}\left(F_{b, m}\right)\right)^{2}(x) d x+C_{11} \int_{\Omega}\left(\mathscr{A}_{L}\left(F_{b, m}\right)\right)^{2}(x) d x\right\}
\end{aligned}
$$


There exists a constant $C>0$ such that

$$
\int_{\Omega}\left(\widetilde{\mathscr{A}}_{L}\left(F_{b, m}\right)\right)^{2}(x) d x \leq \int_{\Omega}\left(\widetilde{\mathscr{A}}_{L}(f)\right)^{2}(x) d x \leq C \int_{\Omega}\left|L^{1 / 2}(f)(x)\right|^{2} d x .
$$

Notice that $S_{L, \alpha}$ is bounded on $L^{p}$ for $1<p<\infty$ and $\alpha>0$ (see [25, Proposition 3.3]), we have

$$
\int_{\Omega}\left(\mathscr{A}_{L}\left(F_{b, m}\right)\right)^{2}(x) d x \leq \int_{\Omega}\left(\mathscr{A}_{L}(f)\right)^{2}(x) d x \leq \int_{\Omega}\left(S_{L, 5 \delta^{-2}}(f)\right)^{2}(x) d x \leq C \int_{\Omega}|f(x)|^{2} d x .
$$

These, combined with (6.12) and (6.4), deduce that

$$
\begin{aligned}
\int_{\Omega}|f(x)|^{2} V(x) d x & \leq C_{12} c_{3} \int_{\Omega}\left|L^{1 / 2} f(x)\right|^{2} d x+C C_{11} \int_{\Omega}|f(x)|^{2} d x \\
& \leq C_{12} c_{3} \lambda^{-1} \int_{\Omega}|\nabla f(x)|^{2} d x+C C_{11} \int_{\Omega}|f(x)|^{2} d x
\end{aligned}
$$

for some constant $C_{12}$ and $\lambda$ is the constant in (6.1). We take $c_{3}=\lambda^{2} /\left(2 C_{12}\right)$ in (6.13) and use (6.1) and (6.3) to obtain

$$
\langle\mathcal{L} f, f\rangle \geq C_{12} c_{3} \lambda^{-1} \int_{\Omega}|\nabla f(x)|^{2} d x-\int_{\Omega}|f(x)|^{2} V(x) d x \geq-C C_{11}\langle f, f\rangle, \quad f \in \operatorname{Dom}(\mathcal{L}) .
$$

Finally, it follows by Lemma 6.2 that

$$
\begin{aligned}
\sigma(\mathcal{L}) & \geq-\sup _{Q} c\left[|Q|^{-1} \Lambda(Q, V)-c_{3} \ell(Q)^{-2}|Q|\right] \\
& \geq-\sup _{B} c_{1}\left[|B|^{-1} \Lambda(B, V)-c_{2} r_{B}^{-2}|B|\right]
\end{aligned}
$$

for some $c_{1}, c_{2}>0$. The proof of Theorem 6.1 is complete.

Acknowledgments. P. Chen was partially supported by NNSF of China 11501583, Guangdong Natural Science Foundation 2016A030313351 and the Fundamental Research Funds for the Central Universities 161gpy45. X. T. Duong is supported by Australian Research Council Discovery Grant DP 140100649. L.C. Wu and L.X. Yan are supported by the NNSF of China, Grant Nos. 11371378 and 11521101).

\section{REFERENCES}

[1] P. Auscher, On necessary and sufficient conditions for $L^{p}$-estimates of Riesz transforms associated to elliptic operators on $\mathbb{R}^{n}$ and related estimates. Mem. Amer. Math. Soc. 186 (2007), no. 871, xviii+75 pp. 3 , 26

[2] P. Auscher, T. Coulhon, X.T. Duong and S. Hofmann, Riesz transform on manifolds and heat kernel regularity. Ann. Sci. École Norm. Sup. 37, (2004), 911-957. 10, 26, 32

[3] P. Auscher, J.M. Martell, Weighted norm inequalities, off-diagonal estimates and elliptic operators. Part I: General operator theory and weights. Adv. Math., 212(2007), 225-276. 26

[4] P. Auscher and E. Russ, Hardy spaces and divergence operators on strongly Lipschitz domain of $\mathbb{R}^{n}$. J. Funct. Anal. 201 (2003), 148-184. 10, 39

[5] F. Bernicot, D. Frey, S. Petermichl, Sharp weighted norm estimates beyond Calderón-Zygmund theory. Anal. PDE. 9 (2016), 1079-1113. 26

[6] A. Calderón, An atomic decomposition of distributions in parabolic $H^{p}$ spaces. Adv. Math., 25 (1977), 216-225. 3, 11

[7] L. Carleson, S.-Y.A. Chang, P. Jones, M. Keel, P. Lax, N. Makarov, D. Sarason, W. Schlag and B. Simon, Thomas H. Wolff (1954-2000). Notices Amer. Math. Soc. 48 (2001), 482-490. 2

[8] S.-Y.A. Chang, J.M. Wilson, T.H. Wolff, Some weighted norm inequalities concerning the Schrödinger operators. Comment. Math. Helv. 60 (1985), 217-246. 2, 3, 5, 13, 25, 39 
[9] S. Chanillo, R.L. Wheeden, $L^{p}$ estimates for fractional integrals and Sobolev inequalities with applications to Schrödinger operators. Comm. Partial Differential Equations 10 (1985), 1077-1116. 5, 39

[10] J. Cheeger, M. Gromov and M. Taylor, Finite propagation speed, kernel estimates for functions of the Laplace operator, and the geometry of complete Riemannian manifolds. J. Differential Geom. 17 (1982), no. 1, 15-53. 8

[11] P. Chen, E.M. Ouhabaz, A. Sikora and L.X. Yan, Restriction estimates, sharp spectral multipliers and endpoint estimates for Bochner-Riesz means. J. Anal. Math. 129 (2016), 219-283. 29

[12] M. Christ, A $T(b)$ theorem with remarks on analytic capacity and the Cauchy integral. Colloq. Math. 60/61 (1990), no. 2, 601-628. 6

[13] R.R. Coifman, G. Weiss, Analyse harmonique hon-commutative sur certains espaces homogènes. Lecture Notes in Math. Vol. 242, Springer, Berlin (1971) 5

[14] T. Coulhon, A. Sikora, Gaussian heat kernel upper bounds via Phragmén-Lindelöf theorem. Proc. Lond. Math. Soc. 96, (2008), 507-544. 8

[15] E.B. Davies, Heat kernels and spectral theory, Cambridge Tracts in Mathematics, 92. Cambridge University Press, Cambridge, Cambridge, 1990. 10, 39

[16] X.T. Duong and A. McIntosh, Singular integral operators with non-smooth kernels on irregular domains, Rev. Mat. Iberoamericana, 15 (1999), 233-265. 26

[17] X.T. Duong, E.M. Ouhabaz and A. Sikora, Plancherel-type estimates and sharp spectral multipliers. J. Funct. Anal., 196 (2002), 443-485. 26, 29, 30

[18] X.T. Duong and L.X. Yan, New function spaces of BMO type, John-Nirenberg inequality, interpolation and applications. Comm. Pure Appl. Math., 58 (2005), 1375-1420. 26

[19] X.T. Duong and L.X. Yan, Duality of Hardy and BMO spaces associated with operators with heat kernel bounds. J. Amer. Math. Soc., 18 (2005), 943-973. 26

[20] J. Dziubański, J. Zienkiewicz, Hardy space $H^{1}$ associated to Schrödinger operator with potential satisfying reverse Hölder inequality. Rev. Mat. Iberoamericana 15 (1999), 279-296. 10

[21] C. Fefferman, The uncertainty principle. Bull. Amer. Math. Soc. (N.S.) 9 (1983), 129-206. 5, 39

[22] R. Fefferman, Harmonic analysis on product spaces. Ann. of Math. (2) 126 (1987), 109-130. 2

[23] R. Fefferman, J. Pipher, Multiparameter operators and sharp weighted inequalities. Amer. J. Math. 119 (1997), 337-369. 2, 26, 27, 30

[24] R.M. Gong, P.Z. Xie, Weighted $L^{p}$ estimates for the area integral associated with self-adjoint operators on homogeneous space. J. Math. Anal. Appl. 393 (2012), 590-604. 4, 30

[25] R.M. Gong, L.X. Yan, Weighted $L^{p}$ estimates for the area integral associated to self-adjoint operators. Manuscripta Math. 144 (2014), 25-49. 3, 4, 30, 39, 42

[26] R.M. Gong, L.X. Yan, Littlewood-Paley and spectral multipliers on weighted $L^{p}$ spaces. J. Geom. Anal. 24 (2014), 873-900. 30, 39

[27] S. Hofmann, G.Z. Lu, D. Mitrea, M. Mitrea and L.X. Yan, Hardy spaces associated to nonnegative self-adjoint operators satisfying Davies-Gaffeney estimates. Mem. Amer. Math. Soc., 214 (2011), no. 1007. 9, 26

[28] T. Hytönen, The sharp weighted bound for general Calderón-Zygmund operators. Ann. of Math. 175 (2012), 14731506.

[29] T. Hytönen, C. Pérez and E. Rela, Sharp reverse Hölder property for $A_{\infty}$ weights on spaces of homogeneous type. J. Funct. Anal. 263 (2012), 3883-3899. 29

[30] T. Hytönen, A. Kairema, Systems of dyadic cubes in a doubling metric space. Colloq. Math. 126 (2012), 1-33. 6, 7

[31] R. Kerman, E.T. Sawyer, Weighted norm inequalities for potentials with applications to Schrödinger operators, Fourier transforms, and Carleson measures. Bull. Amer. Math. Soc. (N.S.) 12 (1985), 112-116. 5,39

[32] A.K. Lerner, Sharp weighted norm inequalities for Littlewood-Paley operators and singular integrals. Adv. Math. 226 (2011), 3912-3926.

[33] A.K. Lerner, F. Nazarov, Intuitive dyadic calculus: the basics. Aavailable at http://arXiv: 1508.05639. 15

[34] P. Li, S.T. Yau, On the parabolic kernel of the Schrödinger operator. Acta Math. 156 (1986), 153-201. 10

[35] E.M. Ouhabaz, Analysis of heat equations on domains. London Math. Soc. Monographs, Vol. 31, Princeton Univ. Press 2005. 10

[36] C. Pérez, On a theorem of Muckenhoupt and Wheeden and a weighted inequality related to Schrödinger operators. Trans. Amer. Math. Soc. 340 (1993), 549-562. 2, 5

[37] Z. Shen, $L^{p}$ estimates for Schrödinger operators with certain potentials. Ann. Inst. Fourier (Grenoble) 45 (1995), 513-546. 10

[38] E. Sawyer, R.L. Wheeden, Weighted inequalities for fractional integrals on Euclidean and homogeneous spaces. Amer. J. Math. 114 (1992), 813-874. 6 
[39] A. Sikora, Riesz transform, Gaussian bounds and the method of wave equation. Math. Z. 247, (2004), 643-662. 8, 9

[40] L. Song, L.X. Yan, A maximal function characterization for Hardy spaces associated to nonnegative self-adjoint operators satisfying Gaussian estimates. Adv. Math. 287 (2016), 463-484. 9

[41] E.M. Stein, Singular integrals and differentiability properties of functions. Princeton Univ. Press, Princeton N.J., 1970. 29

[42] E.M. Stein, Harmonic analysis: Real variable methods, orthogonality and oscillatory integrals, Princeton Univ. Press, Princeton, NJ, 1993. 2

[43] C. Sweezy, L-harmonic functions and the exponential square class. Pacific J. Math. 147 (1991), 187-200. 2

[44] X. Tolsa, BMO, H1, and Calderón-Zygmund operators for non doubling measures. Math. Ann. 319 (2001), 89-149. 25

[45] A. Volberg, Calderón-Zygmund capacities and operators on homogeneous spaces. CBMS Regional Confernces Series in Mathematics, 100 (2003). 25

[46] J.M. Wilson, A sharp inequality for the square function. Duke Math. J. 55, (1987), 879-887. 2, 5

[47] J.M. Wilson, Weighted norm inequalities for the continuous square function. Trans. Amer. Math. Soc. 314 (1989), $661-692.2,5,8,34,37,39$

[48] J. M. Wilson, Weighted Littlewood-Paley theory and exponential-square integrability. Lecture Notes in Math. 1924, Springer, Berlin, 2008. 2, 23, 26, 27, 28, 32, 33, 34, 36, 37, 40

[49] L.C. Wu, The exponential square class on space of homogeneous type. Preprint (2016). 32

[50] K. Yosida, Functional Analysis (Fifth edition). Spring-Verlag, Berlin, 1978. 11, 30

Peng Chen, Department of Mathematics, Sun Yat-sen University, Guangzhou, 510275, P.R. China

E-mail address: chenpeng3@mail.sysu.edu.cn

Xuan Thinh Duong, Department of Mathematics, Macquarie University, NSW 2109, Australia

E-mail address: xuan. duong@mq. edu. au

Liangchuan Wu, Department of Mathematics, Sun Yat-sen University, Guangzhou, 510275, P.R. China

E-mail address: wulchuan@mail2. sysu.edu.cn

Lixin Yan, Department of Mathematics, Sun Yat-sen University, Guangzhou, 510275, P.R. China

E-mail address: mcsylx@mail.sysu.edu.cn 